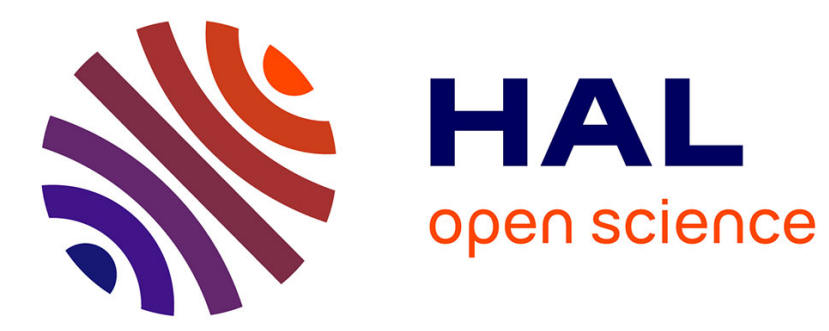

\title{
From Newton's law to the linear Boltzmann equation without cut-off
}

\author{
Nathalie Ayi
}

\section{To cite this version:}

Nathalie Ayi. From Newton's law to the linear Boltzmann equation without cut-off. 2016. hal01279218

\section{HAL Id: hal-01279218 \\ https://hal.science/hal-01279218}

Preprint submitted on 25 Feb 2016

HAL is a multi-disciplinary open access archive for the deposit and dissemination of scientific research documents, whether they are published or not. The documents may come from teaching and research institutions in France or abroad, or from public or private research centers.
L'archive ouverte pluridisciplinaire HAL, est destinée au dépôt et à la diffusion de documents scientifiques de niveau recherche, publiés ou non, émanant des établissements d'enseignement et de recherche français ou étrangers, des laboratoires publics ou privés. 


\title{
From Newton's law to the linear Boltzmann equation without cut-off
}

\author{
Nathalie AYI
}

\begin{abstract}
We provide a rigorous derivation of the linear Boltzmann equation without cut-off starting from a system of particles interacting via a potential with infinite range as the number of particles $N$ goes to infinity under the Boltzmann-Grad scaling.

The main difficulty in our context is that, due to the infinite range of the potential, a nonintegrable singularity appears in the angular collision kernel, making no longer valid the singleuse of Lanford's strategy.

Our proof relies then on a combination of Lanford's strategy, of tools developed recently by Bodineau, Gallagher and Saint-Raymond to study the collision process, and of new duality arguments to study the additional terms associated to the long-range interaction, leading to some explicit weak estimates.
\end{abstract}

\section{Introduction}

In kinetic theory, a gas is described as a physical system constituted of a large number of small particles. The point of view adopted is a statistical one. The fundamental model is the evolution equation for the density of particles of a sufficiently rarefied gas first obtained by Boltzmann in 1872. One of its interesting aspect can be found in the fact that Boltzmann's kinetic equation can be used as an intermediate step in the transition between atomistic and continuous models for gas dynamics as it is mentioned in the famous sixth problem of Hilbert. Consequently, the problem of the derivation of kinetic transport equations from systems of particles is an issue which has been widely studied in the literature, especially in the context of the Boltzmann equation. The historical result in this field is due to Lanford [14] in the case of hard-spheres. He proved the convergence in the low density limit (only for short times). His proof has been completed recently by Gallagher, Saint-Raymond and Texier [10] and by Pulvirenti, Saffirio and Simonella [15] in the case of hard-spheres and short-range potentials.

Theorem 1.1. Consider a system of $N$ particles interacting

- either as hard-spheres of diameter $\varepsilon$

- or via a repulsive potential $\Phi_{\varepsilon}$, with support in $B(0, \varepsilon)$, radial and singular at 0 and such that the scattering of particles can be parametrized by their deflection angle.

Let $f_{0}: \mathbf{R}^{2 d} \rightarrow \mathbf{R}^{+}$be a continuous density of probability such that

$$
\left\|f_{0} \exp \left(\frac{\beta}{2}|v|^{2}\right)\right\|_{L^{\infty}\left(\mathbf{R}_{x}^{d} \times \mathbf{R}_{v}^{d}\right)}<\exp (-\mu)
$$

for some $\beta>0, \mu \in \mathbf{R}$.

Assume that the $N$ particles are initially identically distributed according to $f_{0}$ and "independent" (meaning the correlations vanish asymptotically). Then, there exists some $T^{*}>0$ (depending only on $\beta$ and $\mu$ ) such that, in the Boltzmann-Grad limit $N \rightarrow \infty, N \varepsilon^{d-1}=1$, the distribution function 
of the particles converges uniformly on $\left[0, T^{*}\right] \times \mathbf{R}^{2 d}$ to the solution of the Boltzmann equation

$$
\begin{aligned}
& \partial_{t} f+v \cdot \nabla_{x} f=Q(f, f) \\
& Q(f, f):=\iint_{\mathbf{S}^{d-1} \times \mathbf{R}^{d}}\left[f\left(v^{*}\right) f\left(v_{1}^{*}\right)-f(v) f\left(v_{1}\right)\right] b\left(v-v_{1}, \nu\right) d v_{1} d \nu \\
& v^{*}=v+\nu \cdot\left(v_{1}-v\right) \nu, v_{1}^{*}=v_{1}-\nu \cdot\left(v_{1}-v\right) \nu,
\end{aligned}
$$

with a locally bounded cross-section $b$ depending on $\Phi$ implicitly, and with initial data $f_{0}$. In the case of a hard-sphere interaction, the cross section is given by

$$
b\left(v-v_{1}, \nu\right)=\left(\left(v-v_{1}\right) \cdot \nu\right)_{+} .
$$

Remark 1.1. In the case of hard-spheres for example, the notion of "independence" is translated this way

$$
f_{N \mid t=0}=\frac{1}{\mathcal{Z}_{N}} \prod_{i=1}^{N} f_{0}\left(x_{i}, v_{i}\right) \mathbf{1}_{\mathcal{D}_{\varepsilon}^{N}}
$$

with $\mathcal{D}_{\varepsilon}^{N}:=\left\{\left(x_{1}, v_{1}, \ldots, x_{N}, v_{N}\right) \in \mathbf{R}^{2 d N}|\forall i \neq j,| x_{i}-x_{j} \mid>\varepsilon\right\}$, while $\mathcal{Z}_{n}$ normalizes the integral of $f_{N \mid t=0}$ to 1 .

More recently, Bodineau, Gallagher and Saint-Raymond have been able in [7] to extend this result to any time interval $[0, t]$ with $t \ll \log \log N$ and overcome the difficulty of the short time validity in the particular case of a fluctuation around equilibrium. The key point of their proof is to exploit the maximum principle and establish global uniform a priori bounds for the distribution of particles, and more generally for all finite order marginals of the N-particle distribution.

However, so far the question of the convergence in the case of long-range potentials is still open. Indeed, for a long time Grad's cut-off assumption, which consists in postulating that the collision kernel is integrable with respect to the angular variable (see [11]), was crucial to work even at the level of the kinetic equation. The problem is that, in the case of infinite range forces, whatever the decay at infinity the huge amount of grazing collisions produces a non integrable singularity in the "angular collision kernel". However, recently a couple of breakthroughs have been made regarding the Cauchy theory for this singular equation $[1,2,3,4,5,12]$ inciting us to reconsider this context.

A first partial result in the direction of a derivation from a system of particles with long-range interactions has been obtained by Desvillettes and Pulvirenti [9]. Nevertheless, in their case the long-range interactions are asymptotic, meaning the range of interaction is finite for any fixed $N$ and tends to $\infty$ only in the limit $N \rightarrow \infty$. Moreover, the particle moves in a frozen background, the obstacles being distributed according to some Poisson Law.

A huge number of microscopic contexts has been investigated (see the book by Spohn [16] for a survey on this topic). What we intend to do here is to obtain a complete derivation of the linear Boltzmann equation without cut-off. Our framework will be to consider potentials with strong decay (see Assumption 4.1). Our result is far from being reach near the limit case of Coulomb interaction for which we obtain the Landau equation (see [6] for a derivation of the Landau equation starting from a particle system). Actually, it appears that with our strategy, we can not hope to obtain a result for much less decreasing potential. We end up quite confident about the fact that a different approach is needed to obtain a similar result for more classical potentials such as power law interaction ones for example. Nevertheless, it is a first step into reaching the linear Boltzmann equation without cut-off starting from an infinite range potential at the microscopic level.

\section{Main result and General strategy}

We are interested in describing at the mesoscopic level the behavior of a gas constituted of $\mathrm{N}$ particles. We denote the positions by $X_{N}=\left(x_{1}, \ldots, x_{N}\right)$ and the velocities by $V_{N}=\left(v_{1}, \ldots, v_{N}\right)$. 
We will consider $X_{N}$ in $\left(\mathbf{T}^{d}\right)^{N}$ and $V_{N}$ in $\left(\mathbf{R}^{d}\right)^{N}$ where $\mathbf{T}^{d}$ is the d-dimensional torus. We denote $Z_{N}:=\left(z_{1}, \ldots, z_{N}\right)$ where $z_{i}:=\left(x_{i}, v_{i}\right)$ for $1 \leq i \leq N$. With a slight abuse we say that $Z_{N}$ belongs to $\mathbf{T}^{d N} \times \mathbf{R}^{d N}$ if $X_{N}$ belongs to $\mathbf{T}^{d N}$ and $V_{N}$ to $\mathbf{R}^{d N}$.

The microscopic model is given by : for $i \in \llbracket 1, N \rrbracket$,

$$
\frac{d x_{i}}{d t}=v_{i}, \frac{d v_{i}}{d t}=-\frac{1}{\varepsilon} \sum_{\substack{j=1 \\ j \neq i}}^{N} \nabla \Phi\left(\frac{x_{i}-x_{j}}{\varepsilon}\right)
$$

with $\Phi$ a function satisfying the following assumption:

Assumption 2.1. $\Phi: \mathbf{R}^{d} \rightarrow \mathbf{R}$ is a radial, nonnegative, nonincreasing function which does not vanish, goes to zero at infinity and present a singularity in 0 . Moreover, $\nabla \Phi$ is a Lipschitz function with fast decay (see Assumption 4.1 for more precisions).

The Liouville equation satisfied by the N-particle distribution function $f_{N}$ is

$$
\partial_{t} f_{N}+\sum_{i=1}^{N} v_{i} \cdot \nabla_{x_{i}} f_{N}-\frac{1}{\varepsilon} \sum_{i=1}^{N} \sum_{\substack{j=1 \\ j \neq i}}^{N} \nabla \Phi\left(\frac{x_{i}-x_{j}}{\varepsilon}\right) \cdot \nabla_{v_{i}} f_{N}=0 .
$$

We denote the marginals of order $s$ of $f_{N}$ by $f_{N}^{(s)}\left(t, Z_{s}\right)$ and we define them as follows

$$
f_{N}^{(s)}\left(t, Z_{s}\right):=\int f_{N}\left(t, Z_{N}\right) d z_{s+1} \ldots d z_{N} .
$$

\subsection{Main result}

The framework is the following: we examine a small perturbation around the equilibrium of a fixed number of particles. For the sake of simplicity, we initially perturb only one particle (which will be labeled by 1) with respect to the position $x_{1}$ of the tagged particle. In order to do so, we consider initial data of the form

$$
f_{N}^{0}\left(Z_{N}\right):=M_{N, \beta}\left(Z_{N}\right) \rho^{0}\left(x_{1}\right)
$$

where $\rho^{0}$ is a continuous density of probability on $\mathbf{T}^{d}$ and $M_{N, \beta}$ is the Gibbs measure defined as follows: for $\beta>0$ given

$$
M_{N, \beta}\left(Z_{N}\right):=\frac{1}{\overline{\mathcal{Z}}_{N}}\left(\frac{\beta}{2 \pi}\right)^{d N / 2} \exp \left(-\beta H_{N}\left(Z_{N}\right)\right)
$$

with $H_{N}\left(Z_{N}\right):=\sum_{1 \leq i \leq N} \frac{1}{2}\left|v_{i}\right|^{2}+\sum_{1 \leq i<j \leq N} \Phi\left(\frac{x_{i}-x_{j}}{\varepsilon}\right)$ and

$$
\overline{\mathcal{Z}}_{N}:=\int_{\mathbf{T}^{d N} \times \mathbf{R}^{d N}}\left(\frac{\beta}{2 \pi}\right)^{d N / 2} \exp \left(-\beta H_{N}\left(Z_{N}\right)\right) d Z_{N} .
$$

Indeed, it is well known that stationary solutions of the Liouville equation (2.0.5) provide asymptotically stationary solutions to the Boltzmann hierarchy.

Theorem 2.1. We consider the initial distribution $f_{N}^{0}$ defined in (2.1.1) describing the state of a tagged particle in a background of $N-1$ particles at equilibrium. Under Assumptions 2.1 and 4.1 on the potential, the distribution $f_{N}^{(1)}(t, x, v)$ of the tagged particle converges in $\mathcal{D}^{\prime}\left(\mathbf{T}^{d} \times \mathbf{R}^{d}\right)$ when 
$N$ goes to $\infty$ under the Boltzmann-Grad scaling $N \varepsilon^{d-1}=1$ to $M_{\beta}(v) h(t, x, v)$ where $h(t, x, v)$ is the solution of the linear Boltzmann equation without cut-off

$$
\partial_{t} h+v \cdot \nabla_{x} h=-\iint\left[h(v)-h\left(v^{*}\right)\right] M_{\beta}\left(v_{1}\right) b\left(v-v_{1}\right) d v_{1} d \nu
$$

with initial data $\rho^{0}\left(x_{1}\right)$ and where $M_{\beta}(v):=\left(\frac{\beta}{2 \pi}\right)^{d / 2} \exp \left(-\frac{\beta}{2}|v|^{2}\right), \beta>0$. The cross-section $b$ has a non-integrable singularity depending implicitly on $\Phi$.

Remark 2.1. We notice that because of the translation invariance of $\mathbf{T}^{d}$ and $\rho^{0}$ belonging to $L^{1}\left(\mathbf{T}^{d}\right)$, the distribution $f_{N}^{0}$ is normalized by 1 in $L^{1}\left(\mathbf{T}^{d N} \times \mathbf{R}^{d N}\right)$. Let us also point out that, the tagged particle being the number 1 , due to this distinction $f_{N \mid t=0}$ is symmetric with respect to $z_{2}, \ldots, z_{N}$ but not to $z_{1}$.

\subsection{Series expansion and general convergence strategy}

In order to introduce the necessary notions, we briefly recall the case of hard-spheres.

\subsubsection{Lanford's strategy in the hard-spheres case}

Let us assume in this subsection that the microscopic dynamics is the hard-spheres one. A general strategy consists in using the Green's formula to obtain the following system of equations for $s<N$ which is called the BBGKY hierarchy:

$$
\left(\partial_{t}+\sum_{i=1}^{s} v_{i} \cdot \nabla_{x_{i}}\right) f_{N}^{(s)}\left(t, Z_{s}\right)=\left(\tilde{\mathcal{C}}_{s, s+1} f_{N}^{(s+1)}\right)\left(t, Z_{s}\right)
$$

on $\mathcal{D}_{\varepsilon}^{s}$ with the operator $\tilde{\mathcal{C}}_{s, s+1}$ defining the collision term as follows

$$
\begin{aligned}
& \left(\tilde{\mathcal{C}}_{s, s+1} f_{N}^{(s+1)}\right)\left(Z_{s}\right) \\
& :=(N-s) \varepsilon^{d-1} \sum_{i=1}^{s} \int_{\mathbf{S}^{d-1} \times \mathbf{R}^{d}} f_{N}^{(s+1)}\left(\ldots, x_{i}, v_{i}^{*}, \ldots, x_{i}+\varepsilon \nu, v_{s+1}^{*}\right)\left(\left(v_{s+1}-v_{i}\right) \cdot \nu\right)_{+} d \nu d v_{s+1} \\
& -(N-s) \varepsilon^{d-1} \sum_{i=1}^{s} \int_{\mathbf{S}^{d-1} \times \mathbf{R}^{d}} f_{N}^{(s+1)}\left(\ldots, x_{i}, v_{i}, \ldots, x_{i}+\varepsilon \nu, v_{s+1}\right)\left(\left(v_{s+1}-v_{i}\right) . \nu\right)_{-} d \nu d v_{s+1}
\end{aligned}
$$

where $\mathbf{S}^{d-1}$ denotes the unit sphere in $\mathbf{R}^{d}$, and $v_{i}^{*}$ and $v_{s+1}^{*}$ stand for the pre-collisional velocities for the particles $i$ and $s+1$. Mild solutions of the hierarchy can then be defined by Duhamel's formula:

$$
f_{N}^{(s)}(t)=\mathcal{T}_{s}(t) f_{N}^{(s)}(0)+\int_{0}^{t} \mathcal{T}_{s}\left(t-t_{1}\right) \tilde{\mathcal{C}}_{s, s+1} f_{N}^{(s+1)}\left(t_{1}\right) d t_{1}
$$

where we denote by $\mathcal{T}_{s}$ the group associated to free transport in $\mathcal{D}_{\varepsilon}^{s}$ with specular reflection on the boundary. The key point of Lanford's proof is the iterated Duhamel formula in order to express solutions of the BBGKY hierarchy in terms of a series of operators applied to the initial marginals:

$f_{N}^{(s)}(t)=\sum_{n=0}^{N-s} \int_{0}^{t} \int_{0}^{t_{1}} \ldots \int_{0}^{t_{n-1}} \mathcal{T}_{s}\left(t-t_{1}\right) \tilde{\mathcal{C}}_{s, s+1} \mathcal{T}_{s+1}\left(t_{1}-t_{2}\right) \tilde{\mathcal{C}}_{s+1, s+2} \ldots \mathcal{T}_{s+n}\left(t_{n}\right) f_{N}^{(s+n)}(0) d t_{n} \ldots d t_{1}$.

The Boltzmann series expansion is obtained by taking the formal limit. The asymptotic expression for the collision operator is given by 


$$
\begin{aligned}
& \left(\tilde{\mathcal{C}}_{s, s+1}^{0} g^{(s+1)}\right)\left(Z_{s}\right) \\
& :=\sum_{i=1}^{s} \int_{\mathbf{S}^{d-1} \times \mathbf{R}^{d}} g^{(s+1)}\left(\ldots, x_{i}, v_{i}^{*}, \ldots, x_{i}, v_{s+1}^{*}\right)\left(\left(v_{s+1}-v_{i}\right) \cdot \nu\right)_{+} d \nu d v_{s+1} \\
& -\sum_{i=1}^{s} \int_{\mathbf{S}^{d-1} \times \mathbf{R}^{d}} g^{(s+1)}\left(\ldots, x_{i}, v_{i}, \ldots, x_{i}, v_{s+1}\right)\left(\left(v_{s+1}-v_{i}\right) \cdot \nu\right)_{-} d \nu d v_{s+1}
\end{aligned}
$$

and the iterated Duhamel formula for the Boltzmann equation leads to the following expression

$$
g^{(s)}(t)=\sum_{n \geq 0} \int_{0}^{t} \int_{0}^{t_{1}} \ldots \int_{0}^{t_{n-1}} \mathcal{T}_{s}^{0}\left(t-t_{1}\right) \tilde{\mathcal{C}}_{s, s+1}^{0} \mathcal{T}_{s}^{0}\left({ }_{1}-t_{2}\right) \tilde{\mathcal{C}}_{s+1, s+2}^{0} \ldots \mathcal{T}_{s+n}\left(t_{n}\right) g^{(s+n)}(0) d t_{n} \ldots d t_{1}
$$

where we denote by $\mathcal{T}_{s}^{0}$ the free flow of $s$ particles on $\mathbf{R}^{2 d s}$.

Two steps are then necessary to prove Lanford's result:

- a uniform short time bound for the series expansion associated to the BBGKY hierarchy and the Boltzmann equation,

- the term by term convergence.

Let us be more precise and introduce the notion of pseudo-trajectory to explain the strategy of convergence. We introduce the following notation

$$
\tilde{\mathcal{C}}_{s, s+1}=\sum_{i=1}^{s} \tilde{\mathcal{C}}_{s, s+1}^{+, i}-\tilde{\mathcal{C}}_{s, s+1}^{-, i}
$$

where

$$
\begin{array}{r}
\left(\tilde{\mathcal{C}}_{s, s+1}^{ \pm, i} f_{N}^{(s+1)}\right)\left(Z_{s}\right):=(N-s) \varepsilon^{d-1} \int_{\mathbf{S}^{d-1} \times \mathbf{R}^{d}} f_{N}^{(s+1)}\left(\ldots, x_{i}, v_{i}^{\prime}, \ldots, x_{i}+\varepsilon \nu, v_{s+1}^{\prime}\right) \\
\left(\left(v_{s+1}-v_{i}\right) \cdot \nu\right)_{ \pm} d \nu d v_{s+1}
\end{array}
$$

with $v_{i}^{\prime}=v_{i}^{*}$ and $v_{s+1}^{\prime}=v_{s+1}^{*}$ for $\tilde{\mathcal{C}}_{s, s+1}^{+, i}$ and $v_{i}^{\prime}=v_{i}$ and $v_{s+1}^{\prime}=v_{s+1}$ for $\tilde{\mathcal{C}}_{s, s+1}^{-, i}$. We can do the same for $\tilde{\mathcal{C}}_{s, s+1}^{0}$. We call elementary terms in the series the following elements

$$
\int_{0}^{t} \int_{0}^{t_{1}} \ldots \int_{0}^{t_{n-1}} \mathcal{T}_{s}\left(t-t_{1}\right) \tilde{\mathcal{C}}_{s, s+1}^{j_{1}, m_{1}} \mathcal{T}_{s+1}\left(t_{1}-t_{2}\right) \tilde{\mathcal{C}}_{s+1, s+2}^{j_{2}, m_{2}} \ldots \mathcal{T}_{s+n}\left(t_{n}\right) f_{N}^{(s+n)}(0) d t_{n} \ldots d t_{1}
$$

with $\left(j_{1}, j_{2}, \ldots, j_{n}\right) \in\{+,-\}$ and $m_{i} \in\{1,2, \ldots, s+i-1\}$. Each elementary term has a geometric interpretation as an integral over some pseudo-trajectory.

Definition 2.2. We call pseudo-trajectory associated to the BBGKY hierarchy and the elementary term

$$
\int_{0}^{t} \int_{0}^{t_{1}} \ldots \int_{0}^{t_{n-1}} \mathcal{T}_{s}\left(t-t_{1}\right) \tilde{\mathcal{C}}_{s, s+1}^{j_{1}, m_{1}} \mathcal{T}_{s+1}\left(t_{1}-t_{2}\right) \tilde{\mathcal{C}}_{s+1, s+2}^{j_{2}, m_{2}} \ldots \mathcal{T}_{s+n}\left(t_{n}\right) f_{N}^{(s+n)}(0) d t_{n} \ldots d t_{1}
$$

the following description of the evolution of the positions and the velocities:

- We start at time $t$ with s particles with the configuration $Z_{s} \in \mathbf{T}^{d s} \times \mathbf{R}^{d s}$. We denote by $\hat{\Psi}_{s}$ the backward s-particle flow. For $u \in\left[t_{1}, t\right], Z_{s}(u):=\hat{\Psi}_{s}(u) Z_{s}$.

- The first collision operator $\tilde{\mathcal{C}}_{s, s+1}^{j_{1}, m_{1}}$ is interpreted as the adjunction at time $t_{1}$ of a new particle at $x_{m_{1}}\left(t_{1}\right)+\varepsilon \nu_{s+1}$ for a deflection angle $\nu_{s+1} \in \mathbf{S}^{d-1}$ and a velocity $v_{s+1} \in \mathbf{R}^{d}$. 
- Then $Z_{s+1}$ evolves according to the backward $s+1$-particles flow $\hat{\Psi}_{s+1}$ during the time interval $\left[t_{2}, t_{1}\right]$ starting at $t_{1}$ from

$$
\begin{aligned}
Z_{s+1}\left(t_{1}\right) & =\left(\left\{z_{j}\left(t_{1}\right)\right\}_{j \neq m_{1}},\left(x_{m_{1}}\left(t_{1}\right), v_{m_{1}}\left(t_{1}\right)\right),\left(x_{m_{1}}\left(t_{1}\right)+\varepsilon \nu_{s+1}, v_{s+1}\right)\right) \quad \text { if } j_{1}=- \\
& =\left(\left\{z_{j}\left(t_{1}\right)\right\}_{j \neq m_{1}},\left(x_{m_{1}}\left(t_{1}\right), v_{m_{1}}^{*}\left(t_{1}\right)\right),\left(x_{m_{1}}\left(t_{1}\right)+\varepsilon \nu_{s+1}, v_{s+1}^{*}\right)\right) \quad \text { if } j_{1}=+.
\end{aligned}
$$

- We iterate this procedure by adding a particle labeled $s+i$ at time $t_{i}$ at $x_{m_{i}}\left(t_{i}\right)+\varepsilon \nu_{s+i}$ for a deflection angle $\nu_{s+i} \in \mathbf{S}^{d-1}$ and a velocity $v_{s+i} \in \mathbf{R}^{d}$. The evolution of $Z_{s+i}$ follows the flow of the backward $s+i$-particles flow $\hat{\Psi}_{s+i}$ during the time interval $\left[t_{i+1}, t_{i}\right]$ starting at $t_{i}$ from

$$
\begin{aligned}
Z_{s+i}\left(t_{i}\right) & =\left(\left\{z_{j}\left(t_{i}\right)\right\}_{j \neq m_{i}},\left(x_{m_{i}}\left(t_{i}\right), v_{m_{i}}\left(t_{i}\right)\right),\left(x_{m_{i}}\left(t_{i}\right)+\varepsilon \nu_{s+i}, v_{s+i}\right)\right) \quad \text { if } j_{i}=- \\
& =\left(\left\{z_{j}\left(t_{i}\right)\right\}_{j \neq m_{i}},\left(x_{m_{i}}\left(t_{i}\right), v_{m_{i}}^{*}\left(t_{i}\right)\right),\left(x_{m_{i}}\left(t_{i}\right)+\varepsilon \nu_{s+i}, v_{s+i}^{*}\right)\right) \quad \text { if } j_{i}=+
\end{aligned}
$$

The elementary term can then be rewritten as follows

$$
\begin{gathered}
\varepsilon^{(d-1) n}(N-s)(N-s-1) \ldots(N-s-n+1) \int_{0}^{t} \int_{0}^{t_{1}} \ldots \int_{0}^{t_{n-1}} d t_{n} \ldots d t_{1} \\
\int_{\left(\mathbf{S}^{d-1} \times \mathbf{R}^{d}\right)^{n}} d \nu_{s+1} \ldots \nu_{s+n} d v_{s+1} \ldots d v_{s+n} \prod_{i=1}^{n}\left(\left(v_{s+i}-v_{m_{i}}\left(t_{i}\right)\right) \cdot \nu_{s+i}\right) f_{N}^{0(s+n)}\left(Z_{s+n}(0)\right)
\end{gathered}
$$

where $Z_{s+n}(0)$ is the pseudo-trajectory at time 0 .

We then give the definition of the two notions of collision and recollision.

Definition 2.3. We call a collision the creation of a particle in the process described above and a recollision the event when two particles collide in the flow $\hat{\Psi}_{s+i}$, with $0 \leq i \leq N-s$.

Note that the pseudo-trajectories do not involve physical particles but are a geometric interpretation of the iterated Duhamel formula in terms of a branching process flowing backward in time and determined by

- the collision times $T:=\left(t_{1}, \ldots, t_{n}\right)$ which are interpreted as branching times,

- the labels of the particles involved in the collisions $m:=\left(m_{1}, \ldots, m_{s+n-1}\right)$ from which branching occurs and such that $1 \leq m_{i} \leq i$ for all $i$,

- the coordinate of the initial particles $Z_{s}$ at time $t$,

- the velocities $v_{s+1}, \ldots, v_{s+n}$ in $\mathbf{R}^{d}$ and deflection angles $\nu_{s+1}, \ldots, \nu_{s+i} \in \mathbf{S}^{d-1}$ for each additional particle.

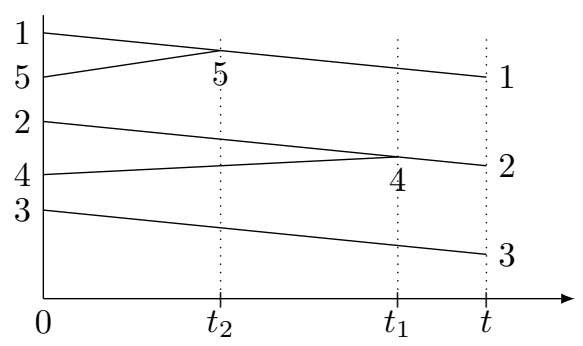

FiguRE 1. Representation of a collision tree associated to the term $\int_{0}^{t} \int_{0}^{t_{1}} \mathcal{T}_{3}^{0}\left(t-t_{1}\right) \tilde{\mathcal{C}}_{3,4}^{j_{1}, 2} \mathcal{T}_{4}^{0}\left(t_{1}-t_{2}\right) \tilde{\mathcal{C}}_{4,5}^{j_{2}, 1} \mathcal{T}_{5}\left(t_{2}\right) f_{N}^{(5)}(0) d t_{2} d t_{1}$ 
Definition 2.4. We call pseudo-trajectory associated to the Boltzmann hierarchy and the elementary term

$$
\int_{0}^{t} \int_{0}^{t_{1}} \ldots \int_{0}^{t_{n-1}} \mathcal{T}_{s}\left(t-t_{1}\right) \tilde{\mathcal{C}}_{s, s+1}^{0, j_{1}, m_{1}} \mathcal{T}_{s+1}\left(t_{1}-t_{2}\right) \tilde{\mathcal{C}}_{s+1, s+2}^{0, j_{2}, m_{2}} \ldots \mathcal{T}_{s+n}\left(t_{n}\right) f_{N}^{(s+n)}(0) d t_{n} \ldots d t_{1}
$$

the following description of the evolution of the positions and the velocities:

we start at time $t$ with $s$ particles with the configuration $Z_{s}^{0} \in \mathbf{T}^{d s} \times \mathbf{R}^{d s}$. The $(s+k)^{\text {th }}$ particle is added at $x_{m_{k}}^{0}\left(t_{k}\right)$ with a velocity $v_{s+k} \in \mathbf{R}^{d}$. Then $Z_{s+k}^{0}$ evolves according to the backward free flow denoted by $\hat{\Psi}_{s+k}^{0}$ during the time interval $\left[t_{k+1}, t_{k}\right]$ until the next creation, starting from

$$
\begin{aligned}
Z_{s+k}^{0}\left(t_{k}\right) & =\left(\left\{z_{j}^{0}\left(t_{k}\right)\right\}_{j \neq m_{k}},\left(x_{m_{k}}^{0}\left(t_{k}\right), v_{m_{k}}\left(t_{k}\right)\right),\left(x_{m_{k}}^{0}\left(t_{k}\right), v_{s+k}\right)\right) \quad \text { if } j_{k}=- \\
& =\left(\left\{z_{j}^{0}\left(t_{k}\right)\right\}_{j \neq m_{k}},\left(x_{m_{k}}^{0}\left(t_{k}^{+}\right), v_{m_{k}}^{*}\left(t_{k}^{+}\right)\right),\left(x_{m_{k}}^{0}\left(t_{k}^{+}\right), v_{s+k}^{*}\right)\right) \quad \text { if } j_{k}=+.
\end{aligned}
$$

The elementary term can then be rewritten as follows

$$
\begin{aligned}
& \int_{0}^{t} \int_{0}^{t_{1}} \ldots \int_{0}^{t_{n-1}} d t_{n} \ldots d t_{1} \\
& \int_{\left(\mathbf{S}^{d-1} \times \mathbf{R}^{d}\right)^{n}} d \nu_{s+1} \ldots \nu_{s+n} d v_{s+1} \ldots d v_{s+n} \prod_{i=1}^{n}\left(\left(v_{s+i}-v_{m_{i}}\left(t_{i}\right)\right) \cdot \nu_{s+i}\right) g^{0(s+n)}\left(Z_{s+n}^{0}(0)\right)
\end{aligned}
$$

where $Z_{s+n}^{0}(0)$ is the Boltzmann pseudo-trajectory at time 0 .

Remark 2.2. The notion of collision is defined similarly as previously as the creation of a particle in the above process. Nevertheless, in the case of the pseudo-trajectory associated to the Boltzmann hierarchy, the particles are points and no recollision occurs in the branching process.

The key point to prove the convergence is actually to prove that the pseudo-trajectories associated to both series can be coupled precisely. Indeed, the differences between the BBGKY series and the Boltzmann series are the prefactors $(N-s) \varepsilon^{d-1}$, the micro-translation $x_{i}+\varepsilon \nu$ when a particle is created in the BBGKY pseudo-trajectory and most importantly the absence of recollisions in the case of the Boltzmann pseudo-trajectories. The two first points are easily dealt with by passing to the limit. The main concern of the proof is then to deal with the third one and prove that outside a geometrical ensemble of vanishing measure, no recollision occurs either for the BBGKY pseudo-trajectories.

\subsubsection{The linear case}

In the original method of Lanford, after a short time (depending on the initial data), the bounding series becomes divergent since possible cancellations between gain and loss terms are completely neglected in this strategy. The idea developed in [7] is to take advantage of the control by stationary solutions to remove the short time limitation. Considering particles living in the torus $\mathbf{T}^{d}$ (and no longer in $\mathbf{R}^{d}$ ) with a perturbation of some equilibrium, Bodineau et al proved the convergence to the linear Boltzmann equation using a slightly different iterative strategy that we will adopt in Section 3.2. The main idea is to bound the number of collisions by working with the so-called collision trees of "controled size".

Definition 2.5. Let us fix a (small) parameter $\tau>0$ and denote $t:=K \tau$ for some large integer $K$ to be fixed later. We split the time interval $[0, t]$ into intervals $\cup_{1 \leq k \leq K}[(k-1) \tau, k \tau]$. We call a collision tree "of controlled size" a collision tree such that it has less than $n_{k}=2^{k}$ branching points on the interval $[t-k \tau, t-(k-1) \tau]$.

We call a collision tree with super-exponential growth a collision tree which does not satisfy the above property. 
The strategy is then to define by iteration what we will call a main term where the collision trees involved are collision trees of "controlled size", i.e. where the trajectories with at least $n_{k}$ collisions during the interval time $[t-k \tau, t-(k-1) \tau]$ are truncated, giving birth to some remainders where collision trees with super-exponential growth are involved. The final result is then obtained by proving the convergence of the main term and the vanishing of the remainders (in other words, proving that the contribution of super-exponential trees is negligible).

\subsubsection{The case of a non cut-off potential}

Let us go back to the long-range interaction case. The issue is the following, no matter how decreasing the potential is taken, a non-integrable singularity in the angular collision kernel appears due to the huge amount of grazing collisions. By grazing collisions, we mean collisions with a very large impact parameter, the impact parameter being the distance of closest approach if the two particles move freely (so concretely, grazing collisions involve colliding particles which are barely deviated).

Example 2.1. In the model case of inverse-power law potentials

$$
\Phi(r)=\frac{1}{r^{s-1}}, s>2 .
$$

the cross-section satisfies

$$
b\left(\left|v-v^{*}\right|, \cos \theta\right)=q(\cos \theta)\left|v-v^{*}\right|^{\gamma}, \gamma=\frac{s-(2 d-1)}{s-1}
$$

where $q$ is a function which is only implicitly defined, locally smooth and has a non-integrable singularity for $\theta \rightarrow 0$ :

$$
(\sin \theta)^{d-2} q(\cos \theta) \sim C \theta^{-1-\alpha}, \alpha>0
$$

(see [8] for more details).

So the strategy consisting in neglecting the cancellations between the gain and the loss terms no longer works, even for a short time. Indeed, separating the gain and the loss terms no longer makes sens since the two integrals diverge in

$$
\iint_{\mathbf{S}^{d-1} \times \mathbf{R}^{d}} f\left(v^{*}\right) f\left(v_{1}^{*}\right) b\left(v-v_{1}^{*}, \nu\right) d v_{1} d \nu-\iint_{\mathbf{S}^{d-1} \times \mathbf{R}^{d}} f(v) f\left(v_{1}\right) b\left(v-v_{1}, \nu\right) d v_{1} d \nu
$$

So, the key point will be to separate the contribution of the long-range interaction from the one of the "moderate-range" interaction by introducing a truncation parameter $R$. The "moderate-range" interaction part should be treated using exactly the same strategy as the one explain above in the hard-spheres case introducing some fictitious boundary at distance $R \varepsilon$ (see [13]). The long-range interaction part should be treated as an additional remainder term which will vanish in the limit provided that $R$ goes to $\infty$. The main difficulty in that last term (which does not appear in the case of a short-range potential) is due to the presence of derivatives acting on the marginals. The strategy will be not to iterate on terms involving derivatives and to adopt a weak approach making the derivatives act on test functions. It is actually the core of our proof to develop new duality arguments to study those additional terms and then establish some weak estimates.

Similarly as previously, we will define the notion of pseudo-trajectories associated to the "moderaterange" interaction part the same way as the ones associated to the BBGKY hierarchy in the hard-spheres case except that the new particle created will be add at a distance $R \varepsilon$ instead of $\varepsilon$. Moreover, the scattering not being instantaneous in the case of a potential, when a particle is added in a post-collisional configuration, the two particles perform a backward scattering during the scattering time denoted $t_{\varepsilon}$. However, in the case of long-range interactions, the notion of recollision needs to be defined since once a particle is created, it actually never stops interacting with its "progenitor" (the particle is added next to). 
Definition 2.6. We call a collision the creation of a particle in the process described in the definition of a pseudo-trajectory and a recollision the event where two particles are at a distance $R \varepsilon$ while it is not a creation of a particle.

Then, as previously, we will conclude by coupling the pseudo-trajectories, proving that they remain close outside a set leading to situations involving recollisions which will be of vanishing measure.

\subsection{The iteration strategy}

Due to the presence of the long-range potential, we artificially truncate the potential by considering truncated marginals $\tilde{f}_{N, R}^{(s)}$ defined as follows

$$
\tilde{f}_{N, R}^{(s)}\left(t, Z_{s}\right):=\int_{\mathbf{T}^{d(N-s)} \times \mathbf{R}^{d(N-s)}} f_{N}\left(t, Z_{s}, z_{s+1}, \ldots, z_{N}\right) \prod_{\substack{1 \leq i \leq s \\ s+1 \leq j \leq N}} \mathbf{1}_{\left\{\left|x_{i}-x_{j}\right|>R \varepsilon\right\}} d Z_{(s+1, N)}
$$

where $d Z_{(s+1, N)}:=d z_{s+1} d z_{s+2} \ldots d z_{N}$.

We consider $\Lambda^{R}$ a smooth function such that

$$
\Lambda^{R}(x)= \begin{cases}1 & \text { if }|x|>R \\ 0 & \text { if }|x|<R-1 .\end{cases}
$$

We will denote $\Phi^{>}(x):=\Phi(x) \Lambda^{R}(x)$ and $\Phi^{<}(x):=\Phi(x)\left(1-\Lambda^{R}(x)\right)$.

Applying Green's formula in a similar way as in [10], we obtain the following BBGKY hierarchy

$$
\begin{aligned}
& \partial_{t} \tilde{f}_{N, R}^{(s)}+\sum_{i=1}^{s} v_{i} \cdot \nabla_{x_{i}} \tilde{f}_{N, R}^{(s)}-\frac{1}{\varepsilon} \sum_{\substack{i, j=1 \\
i \neq j}}^{s} \nabla \Phi^{<}\left(\frac{x_{i}-x_{j}}{\varepsilon}\right) \cdot \nabla_{v_{i}} \tilde{f}_{N, R}^{(s)} \\
& =\frac{1}{\varepsilon} \sum_{\substack{i, j=1 \\
i \neq j}}^{s} \nabla \Phi^{>}\left(\frac{x_{i}-x_{j}}{\varepsilon}\right) \cdot \nabla_{v_{i}} \tilde{f}_{N, R}^{(s)} \\
& +\frac{(N-s)}{\varepsilon} \sum_{i=1}^{s} \int_{\mathbf{T}^{d(N-s)} \times \mathbf{R}^{d(N-s)}} \nabla \Phi\left(\frac{x_{i}-x_{s+1}}{\varepsilon}\right) \cdot \nabla_{v_{i}} f_{N}\left(t, Z_{N}\right) \prod_{\substack{1 \leq l \leq s \\
s+1 \leq k \leq N}} \mathbf{1}_{\left\{\left|x_{l}-x_{k}\right|>R \varepsilon\right\}} d Z_{(s+1, N)} \\
& \quad+\mathcal{C}_{s, s+1} \tilde{f}_{N, R}^{(s+1)}+\mathcal{C}_{s, s+1} \bar{f}_{N, R}^{(s+1)}
\end{aligned}
$$

where for $g_{s+1}: \mathbf{T}^{d(s+1)} \times \mathbf{R}^{d(s+1)} \rightarrow \mathbf{R}$

$$
\mathcal{C}_{s, s+1} g_{s+1}\left(Z_{s}\right)=(N-s) \sum_{i=1}^{s} \int_{S_{R \varepsilon}\left(x_{i}\right) \times \mathbf{R}^{d}}\left(\prod_{\substack{j=1 \\ j \neq i}}^{s} \mathbf{1}_{\left|x_{j}-x_{s+1}\right|>R \varepsilon}\right) \nu^{s+1, i} \cdot\left(v_{s+1}-v_{i}\right)
$$

with $\nu^{s+1, i}=\frac{x_{s+1}-x_{i}}{\left|x_{s+1}-x_{i}\right|}, d \sigma_{i}$ is the surface measure on $S_{R \varepsilon}:=\left\{x \in \mathbf{T}^{d},\left|x-x_{i}\right|=R \varepsilon\right\}$ and

$$
\begin{aligned}
\bar{f}_{N, R}^{(s+1)}\left(t, Z_{s+1}\right):= & \int_{\mathbf{T}^{d(N-(s+1))} \times \mathbf{R}^{d(N-(s+1))}} f_{N}\left(t, Z_{N}\right) \\
& \left(\prod_{\substack{1 \leq k \leq s \\
s+2 \leq l \leq N}} \mathbf{1}_{\left|x_{k}-x_{l}\right|>R \varepsilon}\right)\left(1-\prod_{j=s+2}^{N} \mathbf{1}_{\left|x_{j}-x_{s+1}\right|>R \varepsilon}\right) d Z_{(s+2, N)} .
\end{aligned}
$$


We denote by $H_{s}^{<}$the s-particle Hamiltonian defined as follows

$$
H_{s}^{<}\left(Z_{s}\right):=\sum_{1 \leq i \leq s} \frac{1}{2}\left|v_{i}\right|^{2}+\sum_{1 \leq i<j \leq s} \Phi^{<}\left(\frac{x_{i}-x_{j}}{\varepsilon}\right)
$$

and we notice that $H_{s}^{<}$depends on $\varepsilon$ and $R$.

Remark 2.3. We notice that if the potential is supported in the ball of radius $R$, the equation becomes

$$
\partial_{t} \tilde{f}_{N, R}^{(s)}+\sum_{i=1}^{s} v_{i} \cdot \nabla_{x_{i}} \tilde{f}_{N, R}^{(s)}-\frac{1}{\varepsilon} \sum_{\substack{i, j=1 \\ i \neq j}}^{s} \nabla \Phi\left(\frac{x_{i}-x_{j}}{\varepsilon}\right) \cdot \nabla_{v_{i}} \tilde{f}_{N, R}^{(s)}=\mathcal{C}_{s, s+1} \tilde{f}_{N, R}^{(s+1)}+\mathcal{C}_{s, s+1} \bar{f}_{N, R}^{(s+1)}
$$

which is perfectly consistent with the expression found in Section 9.4 of [10], the only difference being the expression of the second term of the right-hand side of (2.3.6). Let us investigate it. The situation described is the following: we have a collision between a particle $i \in\{1, \ldots, s\}$ and particle $s+1$, both being at a distance R $\varepsilon$. Moreover, the presence of $\left(1-\prod_{j=s+2}^{N} \mathbf{1}_{\left|x_{j}-x_{s+1}\right|>R \varepsilon}\right)$ in $\bar{f}_{N, R}^{(s+1)}$ implies that there exists at least one $j_{0} \in\{s+2, \ldots, N\}$ such that particle $s+1$ and particle $j_{0}$ are at a distance lower or equal to Re. This means that we are in presence of multiple simultaneous collisions. The strategy in [10] is to decompose this term and write it using clusters (clusters describe all kind of situations that can happen when multiple collisions are involved) and to force the appearance of truncated marginals of higher order. We can then obtain a closed system on which an argument of Cauchy-Kowalewskaya's type can be applied. There is no need to do that in our case because the control on the marginals is obtained directly from the maximum principle thanks to some a priori estimates (see Section 4).

Mild solutions of the BBGKY hierarchy are thus defined by Duhamel's formula

$$
\begin{aligned}
\tilde{f}_{N, R}^{(s)}\left(t, Z_{s}\right)= & \mathcal{S}_{s}(t) \tilde{f}_{N, R}^{(s)}\left(0, Z_{s}\right) \\
& +\frac{1}{\varepsilon} \sum_{\substack{i, j=1 \\
i \neq j}}^{t} \int_{0}^{t} \mathcal{S}_{s}\left(t-t_{1}\right)\left[\nabla \Phi^{>}\left(\frac{x_{i}-x_{j}}{\varepsilon}\right) \cdot \nabla_{v_{i}} \tilde{f}_{N, R}^{(s)}\right]\left(t_{1}, Z_{s}\right) d t_{1} \\
& +\frac{(N-s)}{\varepsilon} \sum_{i=1}^{s} \int_{0}^{t} \mathcal{S}_{s}\left(t-t_{1}\right)\left[\int_{\mathbf{T}^{d(N-s)} \times \mathbf{R}^{d(N-s)}} \nabla \Phi\left(\frac{x_{i}-x_{s+1}}{\varepsilon}\right) \cdot \nabla_{v_{i}} f_{N}\right. \\
& \left.\prod_{\substack{1 \leq l \leq s \\
s+1 \leq k \leq N}} \mathbf{1}_{\left\{\left|x_{l}-x_{k}\right|>R \varepsilon\right\}} d Z_{(s+1, N)}\right]\left(t_{1}, Z_{s}\right) d t_{1} \\
& +\int_{0}^{t} \mathcal{S}_{s}\left(t-t_{1}\right) \mathcal{C}_{s, s+1} \tilde{f}_{N, R}^{(s+1)}\left(t_{1}, Z_{s}\right) d t_{1} \\
& +\int_{0}^{t} \mathcal{S}_{s}\left(t-t_{1}\right) \mathcal{C}_{s, s+1} \bar{f}_{N, R}^{(s+1)}\left(t_{1}, Z_{s}\right) d t_{1}
\end{aligned}
$$

denoting by $\mathcal{S}_{s}$ the group associated to the solution operator

$$
\mathcal{S}_{s}(t): f \in \mathcal{C}^{0}\left(\mathbf{T}^{d s} \times \mathbf{R}^{d s} ; \mathbf{R}\right) \mapsto f\left(\Psi_{s}(-t, .)\right) \in \mathcal{C}^{0}\left(\mathbf{T}^{d s} \times \mathbf{R}^{d s} ; \mathbf{R}\right)
$$

where $\Psi_{s}(t)$ is the s-particle Hamiltonian flow associated to $H_{s}^{<}$. We notice that $\mathcal{S}_{s}$ depends on $\varepsilon$ and $R$.

Before explaining the iteration strategy, let us point out four possible obstacles to the convergence:

- the very long-range interactions, 
- clusters (or multiple simultaneous interactions),

- the presence of recollisions,

- a super-exponential collision process.

So the strategy will be to iterate Duhamel's formula on a term where none of those four situations happens. The other terms where at least one those four situations happen will give remainders and we will prove that they vanish in the limit.

Let us go back to (2.3.7). It seems then obvious that we will not iterate the Duhamel formula on the second and third terms of the right-hand side or the last one because they respectively are associated to the long-range interaction part and clusters. Moreover, two of them involve vderivatives. So those terms will create remainders. The idea will be then to split the fourth term into two terms with one where no recollision happens. Finally on this recollision free term, we will control the number of collisions by again splitting it into two terms, one for which the number of collisions is super-exponential (which will give the last remainder) and another one. On this final term, none of the four obstacles being involved, we will iterate the Duhamel formula and so on.

Structure of the paper. The main theorem will actually be proved by steps: first proving that the difference between the BBGKY first marginal and the solution of the Boltzmann with cut-off hierarchy converges to 0 when passing to the limit on the truncation parameter in Section 8 , second, passing to the same limit, proving the convergence of the solution of the Boltzmann with cut-off equation to the solution of the Boltzmann without cut-off equation in Section 9. The core of our study consists actually in proving that the remainders built in the elaboration of the expression of the first marginal in Section 3 vanish asymptotically. It will be done in Section 7. The main innovation in this paper is then the treatment of the remainders associated to the long-range part and will be investigated in Section 4. Moreover, due to the presence of those terms, the treatment of recollisions has to be slightly different as we will see it in Section 6. Other remainders are treated in Section 5.

\section{Expression of the marginals}

In this section, our aim is to build the main term and to get an expression for it and for the remainders. As we mentioned in the previous section, since we choose to iterate on the fourth term of the right-hand side of (2.3.7) where the event of very long-range interactions and clusters are excluded, the only thing left to do is to get rid technically of the recollisions and control the number of collisions. For the first challenge, the idea is to remove a geometrical ensemble outside of which no recollision occurs. Regarding the second one, the key will be to apply the pruning process developed in [7]. Though those techniques are interlocked in the iteration process, we will first present them separately for more clarity.

\subsection{Elimination of recollisions in the iteration strategy}

It can be proved that outside a geometrical ensemble that we will call geom $(s+k), s+k$ particles will not undergo a recollision (see Section 6.1). Though we will construct properly this set by an inductive method later, let us here mention the properties that will be needed in the iteration. We fix the parameters $2^{K+1} R \varepsilon \ll \varepsilon_{0} \ll \min (\delta E, 1)$ with $K$ some large integer, $\delta>0, E>0$ parameters to be fixed later. Let us introduce the notion of good configuration.

Definition 3.1. The set of good configuration $\mathcal{G}_{k}\left(\varepsilon_{0}\right)$ is defined as follows:

$$
\mathcal{G}_{k}\left(\varepsilon_{0}\right):=\left\{Z_{k} \in \mathbf{T}^{d k} \times \mathbf{R}^{d k} \mid \forall u \in[0, t] \forall i \neq j d\left(x_{i}-u v_{i}, x_{j}-u v_{j}\right) \geq \varepsilon_{0}\right\} .
$$


The set geom $(s+k)$, a subset of $\mathbf{S}^{d-1} \times \mathbf{R}^{d}$, satisfies the following property. We consider $s+k$ particles such that all their velocities are bounded by $E$. Since the construction of $\operatorname{geom}(s+k)$ is iterative, we assume that before the creation of the $(s+k)^{t h}$-particle, the $s+k-1$ particles are in a good configuration. Then when we add the $(s+k)^{t h}$-particle. After a delay $\delta$, outside the ensemble $\operatorname{geom}(s+k)$, the $s+k$ particles are in a good configuration. A fortiori, they do not undergo recollisions and the transport $\Psi_{s+k}$ coincides with the free flow.

This property implies that in the iteration strategy, we will need to get rid of large velocities and to separate the collisions by a duration of at least $\delta$ to make sure that at each step the particles are in a good configuration. To deal with the large velocities, we use the classical method which consists in cutting off the energy of the system. We consider $\chi^{E^{2}}$ a smooth function such that

$$
\chi^{E^{2}}(y)= \begin{cases}1 & \text { if }|y| \leq E^{2} \\ 0 & \text { if }|y| \geq E^{2}+1\end{cases}
$$

By analogy with the indicator function, we will then abusively denote $\chi^{E^{2}}\left(H_{k}\left(Z_{k}\right)\right)$ by $\chi_{\left\{H_{k}\left(Z_{k}\right) \leq E^{2}\right\}}$ for all integer $k$.

We point out that because of the presence of new terms due to the long-range part of the potential, the strategy of iteration will be slightly different from the one developed in the previous papers. Indeed, the truncations mentioned above must be done at each iteration of the Duhamel formula, and as we will see in Section 4, we need to introduce an additional truncation on small relative velocities in order to deal more easily with the new terms. Then we consider $\chi^{\eta}$ a smooth function such that

and we define $\chi_{\left\{\forall i \in\{1, \ldots, k\},\left|v_{i}-v_{k+1}\right| \geq \eta\right\}}:=\prod_{i=1}^{k} \chi^{\eta}\left(v_{i}-v_{k+1}\right)$.

Finally, we define $\chi_{g e o m}(k)$ as follows

$$
\chi_{\text {geom }(k)}:=\mathbf{1}_{\text {geom }(k)} * \alpha_{\varepsilon}
$$

where $\alpha_{\varepsilon}$ is an approximation of the identity on $\mathbf{S}^{d-1} \times \mathbf{R}^{d}$.

Let us make one iteration. We start from (2.3.7) and we want to iterate on the fourth term of the right-hand side but first we need to prepare it by separating the collisions by a duration of $\delta$, getting rid of large velocities, remove the recollisions and the small relative velocities. So we obtain the following expression:

$$
\begin{aligned}
& \int_{0}^{t} \mathcal{S}_{s}\left(t-t_{1}\right) \mathcal{C}_{s, s+1} \tilde{f}_{N, R}^{(s+1)}\left(t_{1}, Z_{s}\right) d t_{1} \\
& =\int_{t-\delta}^{t} \mathcal{S}_{s}\left(t-t_{1}\right) \mathcal{C}_{s, s+1} \tilde{f}_{N, R}^{(s+1)}\left(t_{1}, Z_{s}\right) d t_{1} \\
& +\int_{0}^{t-\delta} \mathcal{S}_{s}\left(t-t_{1}\right) \mathcal{C}_{s, s+1}\left(1-\chi_{\left\{H_{s+1}\left(Z_{s+1}\right) \leq E^{2}\right\}}\right) \tilde{f}_{N, R}^{(s+1)}\left(t_{1}, Z_{s}\right) d t_{1} \\
& +\int_{0}^{t-\delta} \mathcal{S}_{s}\left(t-t_{1}\right) \mathcal{C}_{s, s+1} \chi_{\left\{H_{s+1}\left(Z_{s+1}\right) \leq E^{2}\right\}} \chi_{g e o m(s+1)} \tilde{f}_{N, R}^{(s+1)}\left(t_{1}, Z_{s}\right) d t_{1} \\
& +\int_{0}^{t-\delta} \mathcal{S}_{s}\left(t-t_{1}\right) \mathcal{C}_{s, s+1} \chi_{\left\{H_{s+1}\left(Z_{s+1}\right) \leq E^{2}\right\}}\left(1-\chi_{g e o m(s+1)}\right)\left(1-\chi_{\left\{\forall i \in\{1, \ldots, s\},\left|v_{i}-v_{s+1}\right| \geq \eta\right\}}\right) \\
& +\int_{0}^{t-\delta} \mathcal{f}_{N}\left(t-t_{1}\right) \mathcal{C}_{s, s+1} \chi_{\left\{H_{s+1}\left(Z_{s+1}\right) \leq E^{2}\right\}}\left(1-\chi_{\text {geom }(s+1)}\right) \chi_{\left\{\forall i \in\{1, \ldots, s\},\left|v_{i}-v_{s+1}\right| \geq \eta\right\}} \\
& \tilde{f}_{N, R}^{(s+1)}\left(t_{1}, Z_{s}\right) d t_{1} .
\end{aligned}
$$


The fifth term is now ready for the next step. We replace $\tilde{f}_{N, R}^{(s+1)}\left(t_{1}, Z_{s}\right)$ in (3.1.5) by its expression given by Duhamel's formula. Then we identify the new term on which we want to iterate which does not involve very-long range interactions or clusters, prepare it the same way and so on.

From now on, for more concision, let us denote $\chi_{\left\{H_{k}\left(Z_{k}\right) \leq E^{2}\right\}}$ just by $\chi_{H_{k}}$ and $\chi_{\left\{\forall i \in\{1, \ldots, k\},\left|v_{i}-v_{k+1}\right| \geq \eta\right\}}$ by $\chi_{\eta_{k+1}}$. We define the following operators:

$$
\begin{aligned}
& Q_{s, s}(t):=\mathcal{S}_{s}(t) \\
& Q_{s, s+n}(t):=\int_{0}^{t-\delta} \int_{0}^{t_{1}-\delta} \ldots \int_{0}^{t_{n-1}-\delta} \mathcal{S}_{s}\left(t-t_{1}\right) \mathcal{C}_{s, s+1} \chi_{H_{s+1}}\left(1-\chi_{g e o m}(s+1)\right) \chi_{\eta_{s+1}} \ldots \\
& \ldots \mathcal{S}_{s+n-1}\left(t_{n-1}-t_{n}\right) \mathcal{C}_{s+n-1, s+n} \chi_{H_{s+n}}\left(1-\chi_{g e o m(s+n)}\right) \chi_{\eta_{s+n}} \mathcal{S}_{s+n}\left(t_{n}\right) d t_{n} \ldots d t_{1} .
\end{aligned}
$$

We iterate $m$ times as explained above. Doing the change of variables, for $i=1, \ldots, n, t_{i}^{\prime}=t_{i}-t_{n+1}$, $t_{n+1}^{\prime}=t_{n+1}$ along with a use of Fubini's theorem, we obtain the following expansion:

$$
\tilde{f}_{N, R}^{(s)}\left(t, Z_{s}\right)=\sum_{n=0}^{m} Q_{s, s+n}(t) \tilde{f}_{N, R}^{(s)}\left(0, Z_{s}\right)+r_{s, m+1}\left(0, t, Z_{s}\right)
$$

where

$$
\begin{aligned}
& r_{s, m+1}\left(0, t, Z_{s}\right):=r_{s, m+1}^{\text {Pot }, a}\left(0, t, Z_{s}\right)+r_{s, m+1}^{\text {Pot }, b}\left(0, t, Z_{s}\right)+r_{s, m+1}^{\text {Clu }}\left(0, t, Z_{s}\right)+r_{s, m+1}^{\text {Tim }}\left(0, t, Z_{s}\right) \\
& \quad+r_{s, m+1}^{\text {Ener }}\left(0, t, Z_{s}\right)+r_{s, m+1}^{\text {Recoll }}\left(0, t, Z_{s}\right)+r_{s, m+1}^{\text {Relat.Vel. }}\left(0, t, Z_{s}\right)+Q_{s, s+m+1}(t) \tilde{f}_{N, R}^{(s+m+1)}\left(t_{m+1}, Z_{s}\right)
\end{aligned}
$$

with the following respective definitions:

- for the remainders associated to the very long-range interaction part

$$
\begin{aligned}
r_{s, m+1}^{P o t, a}\left(0, t, Z_{s}\right):=\sum_{n=0}^{m} \int_{0}^{t-n \delta} & Q_{s, s+n}\left(t-t_{n+1}\right) \\
& \frac{1}{\varepsilon} \sum_{\substack{i, j=1 \\
i \neq j}}^{s+n}\left[\nabla \Phi^{>}\left(\frac{x_{i}-x_{j}}{\varepsilon}\right) \cdot \nabla_{v_{i}} \tilde{f}_{N, R}^{(s+n)}\right]\left(t_{n+1}, Z_{s}\right) d t_{n+1}
\end{aligned}
$$

and

$$
\begin{aligned}
& r_{s, m+1}^{P o t, b}\left(0, t, Z_{s}\right):=\sum_{n=0}^{m} \int_{0}^{t-n \delta} Q_{s, s+n}\left(t-t_{n+1}\right) \frac{(N-(s+n))}{\varepsilon} \\
& \sum_{i=1}^{s+n}\left[\int_{\mathbf{T}^{d(N-(s+n))} \times \mathbf{R}^{d(N-(s+n))}} \nabla \Phi\left(\frac{x_{i}-x_{s+n+1}}{\varepsilon}\right) \cdot \nabla_{v_{i}} f_{N}\right. \\
&\left.\prod_{\substack{1 \leq l \leq s+n \\
s+n+1 \leq k \leq N}} \mathbf{1}_{\left\{\left|x_{l}-x_{k}\right|>R \varepsilon\right\}} d Z_{(s+n, N)}\right]\left(t_{n+1}, Z_{s}\right) d t_{n+1},
\end{aligned}
$$

- for the remainders associated to clusters

$$
r_{s, m+1}^{C l u}\left(0, t, Z_{s}\right):=\sum_{n=0}^{m} \int_{0}^{t-n \delta} Q_{s, s+n}\left(t-t_{n+1}\right) \mathcal{C}_{s+n, s+n+1} \bar{f}_{N, R}^{(s+n+1)}\left(t_{n+1}, Z_{s}\right) d t_{n+1}
$$


Indeed, the domain of integration for those terms regarding the times is $\left\{0 \leq t_{n+1} \leq t_{n} \leq t_{n-1}-\delta \leq\right.$ $\left.t_{n-2}-2 \delta \leq \cdots \leq t_{1}-(n-1) \delta \leq t-n \delta\right\}$.

We introduce the following second operator

$$
\begin{aligned}
& Q_{s, s+n}^{\delta}(t):=\int_{0}^{t-\delta} \int_{0}^{t_{1}-\delta} \cdots \int_{0}^{t_{n-2}-\delta} \int_{0}^{\delta} \mathcal{S}_{s}\left(t-t_{1}\right) \mathcal{C}_{s, s+1} \chi_{H_{s+1}}\left(1-\chi_{\text {geom }(s+1)}\right) \chi_{\eta_{s+1}} \ldots \\
& \ldots \mathcal{S}_{s+n-1}\left(t_{n-1}-t_{n}\right) \mathcal{C}_{s+n-1, s+n} \chi_{H_{s+n}}\left(1-\chi_{g e o m(s+n)}\right) \chi_{\eta_{s+n}} \mathcal{S}_{s+n}\left(t_{n}\right) d t_{n} \ldots d t_{1}
\end{aligned}
$$

The remainders where the two last collisions are separated in time by less than $\delta$ is defined as follows

$$
r_{s, m+1}^{T i m}\left(0, t, Z_{s}\right):=\sum_{n=0}^{m} \int_{0}^{t-n \delta} Q_{s, s+n}^{\delta}\left(t-t_{n+1}\right) \mathcal{C}_{s+n, s+n+1} \tilde{f}_{N, R}^{(s+n+1)}\left(t_{n+1}, Z_{s}\right) d t_{n+1}
$$

which is consistent with the domain of integration regarding the times of this term $\left\{0 \leq t_{n}-\delta \leq\right.$ $t_{n+1} \leq t_{n}$ and $\left.0 \leq t_{n} \leq t_{n-1}-\delta \leq t_{n-2}-2 \delta \leq \cdots \leq t_{1}-(n-1) \delta \leq t-n \delta\right\}$.

Finally, we define

- the remainders corresponding to situations where the energy of the system is not bounded

$$
\begin{array}{r}
r_{s, m+1}^{\text {Ener }}\left(0, t, Z_{s}\right):=\sum_{n=0}^{m} \int_{0}^{t-(n+1) \delta} Q_{s, s+n}\left(t-t_{n+1}\right) \mathcal{C}_{s+n, s+n+1} \\
\left(1-\chi_{H_{s+n+1}}\right) \tilde{f}_{N, R}^{(s+n+1)}\left(t_{n+1}, Z_{s}\right) d t_{n+1},
\end{array}
$$

- the remainders associated to the possible recollisions

$$
\begin{array}{r}
r_{s, m+1}^{R e c o l l}\left(0, t, Z_{s}\right):=\sum_{n=0}^{m} \int_{0}^{t-(n+1) \delta} Q_{s, s+n}\left(t-t_{n+1}\right) \mathcal{C}_{s+n, s+n+1} \chi_{H_{s+n+1}} \chi_{g e o m(s+n+1)} \\
\tilde{f}_{N, R}^{(s+n+1)}\left(t_{n+1}, Z_{s}\right) d t_{n+1},
\end{array}
$$

- the remainders where the lower bound for all the relative velocities no longer holds

$$
\begin{array}{r}
r_{s, m+1}^{\text {Relat.Vel. }}\left(0, t, Z_{s}\right):=\sum_{n=0}^{m} \int_{0}^{t-(n+1) \delta} Q_{s, s+n}\left(t-t_{n+1}\right) \mathcal{C}_{s+n, s+n+1} \chi_{H_{s+n+1}}\left(1-\chi_{g e o m(s+n+1)}\right) \\
\left(1-\chi_{\eta_{s+n+1}}\right) \tilde{f}_{N, R}^{(s+n+1)}\left(t_{n+1}, Z_{s}\right) d t_{n+1}, \quad(3.1 .16)
\end{array}
$$

the domain of integration regarding the times of those terms being $\left\{0 \leq t_{n+1} \leq t_{n}-\delta \leq t_{n-1}-2 \delta \leq\right.$ $\left.\cdots \leq t_{1}-n \delta \leq t-(n+1) \delta\right\}$.

\subsection{Control of the growth of collision trees in the iteration strategy}

As mentioned previously, one of the key point to get the convergence is to work with collision trees of "controlled size". We recall that, as mentioned in Definition 2.5, a collision tree "of controlled size" is a collision tree such that it has less than $n_{k}=2^{k}$ branch points on the interval $[t-k \tau, t-(k-1) \tau]$.

For the sake of completeness, we will recall the pruning process in the hard-spheres case. As seen in Section 2.2.1, iterating the Duhamel formula $N-s$ times we get 
$f_{N}^{(s)}(t)=\sum_{n=0}^{N-s} \int_{0}^{t} \int_{0}^{t_{1}} \ldots \int_{0}^{t_{n-1}} \mathcal{T}_{s}\left(t-t_{1}\right) \tilde{\mathcal{C}}_{s, s+1} \mathcal{T}_{s+1}\left(t_{1}-t_{2}\right) \tilde{\mathcal{C}}_{s+1, s+2} \ldots \mathcal{T}_{s+n}\left(t_{n}\right) f_{N}^{(s+n)}(0) d t_{n} \ldots d t_{1}$

As our main result concerns the first marginal, we start by using this formula with $s=1$ but this time on the time interval $[t-\tau, t]$ instead of $[0, t]$ and by iterating $n_{1}-1$ times instead of $N-s$ :

$$
f_{N}^{(1)}(t)=\sum_{j_{1}=0}^{n_{1}-1} \bar{Q}_{1,1+j_{1}}(\tau) f_{N}^{\left(1+j_{1}\right)}(t-\tau)+R_{1, n_{1}}(t-\tau, t)
$$

where the term $R_{1, n_{1}}$ corresponds to pseudo-trajectories with at least $n_{1}$ collisions

$$
R_{1, n_{1}}\left(t^{\prime}, t\right):=\sum_{p=n_{1}}^{N-1} \bar{Q}_{1,1+p}\left(t-t^{\prime}\right) f_{N}^{(1+p)}\left(t^{\prime}\right)
$$

and $\bar{Q}$ is defined in the hard-spheres case in a quite similar way as $Q$ in our case. More generally, $R_{k, n}$ stands for

$$
R_{k, n}\left(t^{\prime}, t\right):=\sum_{p=n}^{N-k} \bar{Q}_{k, k+p}\left(t-t^{\prime}\right) f_{N}^{(k+p)}\left(t^{\prime}\right)
$$

and this term described trajectories originating at $k$ points at time $t$ and involving at least $n$ collisions during the time span $t-t^{\prime}$.

The idea in the pruning process is that $n_{k}$ being chosen equal to $2^{k}$ the number of collisions involved in the term $R_{l, n_{k}}$ is super-exponential. Such a behavior should be atypical and it can be proved that those terms vanish when passing to the limit.

Let us go back to our pruning process. We can iterate the Duhamel formula in the first term of the right-hand side of (3.2.2) this time on the interval $[t-2 \tau, t-\tau]$ and truncating the contributions with more than $n_{2}$ collisions. We get

$$
\begin{aligned}
f_{N}^{(1)}(t)=\sum_{j_{1}=0}^{n_{1}-1} \sum_{j_{2}=0}^{n_{2}-1} \bar{Q}_{1,1+j_{1}}(\tau) & \bar{Q}_{1+j_{1}, 1+j_{1}+j_{2}}(\tau) f_{N}^{\left(1+j_{1}+j_{2}\right)}(t-2 \tau) \\
& +R_{1, n_{1}}(t-\tau, t)+\sum_{j_{1}=0}^{n_{1}-1} \bar{Q}_{1,1+j_{1}}(\tau) R_{1+j_{1}, n_{2}}(t-2 \tau, t-\tau) .
\end{aligned}
$$

Iterating this procedure $K$ times and truncating the trajectories with at least $n_{k}$ collisions during the time interval $[t-k \tau, t-(k-1) \tau]$, we finally get

$$
f_{N}^{(1)}(t)=f_{N}^{(1, K)}(t)+R_{N}^{K}(t)
$$

where denoting $J_{0}:=1, J_{k}:=1+j_{1}+\cdots+j_{k}$,

$$
f_{N}^{(1, K)}(t):=\sum_{j_{1}=0}^{n_{1}-1} \cdots \sum_{j_{K}=0}^{n_{K}-1} \bar{Q}_{1, J_{1}}(\tau) \bar{Q}_{J_{1}, J_{2}}(\tau) \ldots \bar{Q}_{J_{K-1}, J_{K}}(\tau) f_{N}^{0\left(J_{K}\right)}
$$

and

$$
R_{N}^{K}(t):=\sum_{k=1}^{K} \sum_{j_{1}=0}^{n_{1}-1} \ldots \sum_{j_{k-1}=0}^{n_{k-1}-1} \bar{Q}_{1, J_{1}}(\tau) \ldots \bar{Q}_{J_{k-2}, J_{k-1}}(\tau) R_{J_{k-1}, n_{k}}(t-k \tau, t-(k-1) \tau) .
$$




\subsection{Final expression of the first marginal}

Let us go back to our problem. The idea will be to combine these two methods in order to construct the main term and the remainders. Let us explain how specifically.

- First, we start on the time interval $[t-\tau, t]$. We apply Duhamel's formula for the first marginal and get rid of the recollisions by iterating the process explain in Section 3.1. In order to apply the pruning process, we iterate this process $n_{1}-1$ times. Then we get

$$
\tilde{f}_{N, R}^{(1)}(t)=\sum_{j_{1}=0}^{n_{1}-1} Q_{1,1+j_{1}}(\tau) \tilde{f}_{N, R}^{\left(1+j_{1}\right)}(t-\tau)+r_{1, n_{1}}(t-\tau, t)
$$

with $r_{1, n_{1}}(t-\tau, t)$ defined in Section 3.1.

- Then we work on the time interval $[t-2 \tau, t-\tau]$ with the marginal $\tilde{f}_{N, R}^{\left(1+j_{1}\right)}(t-\tau)$. As previously, we apply Duhamel's formula and get rid of the recollisions by iterating $n_{2}-1$ times the process of Section 3.1. We get

$$
\tilde{f}_{N, R}^{\left(1+j_{1}\right)}(t-\tau)=\sum_{j_{2}=0}^{n_{2}-1} Q_{1+j_{1}, 1+j_{1}+j_{2}}(\tau) \tilde{f}_{N, R}^{\left(1+j_{1}+j_{2}\right)}(t-2 \tau)+r_{1+j_{1}, n_{2}}(t-2 \tau, t-\tau) .
$$

- Finally, we replace $\tilde{f}_{N, R}^{\left(1+j_{1}\right)}(t-\tau)$ by the above expression in (3.3.1) and we get

$$
\begin{aligned}
\tilde{f}_{N, R}^{(1)}(t)=\sum_{j_{1}=0}^{n_{1}-1} \sum_{j_{2}=0}^{n_{2}-1} Q_{1,1+j_{1}}(\tau) Q_{1+j_{1}, 1+j_{1}+j_{2}}(\tau) \tilde{f}_{N, R}^{\left(1+j_{1}+j_{2}\right)}(t-2 \tau) \\
+\sum_{j_{1}=0}^{n_{1}-1} Q_{1,1+j_{1}}(\tau) r_{1+j_{1}, n_{2}}(t-2 \tau, t-\tau)+r_{1, n_{1}}(t-\tau, t) .
\end{aligned}
$$

- We iterate this procedure $K$ times and we finally obtain the following expression

$$
\tilde{f}_{N, R}^{(1)}(t)=\tilde{f}_{N, R}^{(1, K)}(t)+r_{N}^{K}(t)
$$

where

$$
\tilde{f}_{N, R}^{(1, K)}(t):=\sum_{j_{1}=0}^{n_{1}-1} \cdots \sum_{j_{K}=0}^{n_{K}-1} Q_{1, J_{1}}(\tau) Q_{J_{1}, J_{2}}(\tau) \ldots Q_{J_{K-1}, J_{K}}(\tau) \tilde{f}_{N, R}^{0\left(J_{K}\right)}
$$

and

$$
r_{N}^{K}(t):=\sum_{k=1}^{K} \sum_{j_{1}=0}^{n_{1}-1} \ldots \sum_{j_{k-1}=0}^{n_{k-1}-1} Q_{1, J_{1}}(\tau) \ldots Q_{J_{k-2}, J_{k-1}}(\tau) r_{J_{k-1}, n_{k}}(t-k \tau, t-(k-1) \tau) .
$$

\section{Terms associated to the long-range part of the potential}

In this section, our aim will be to deal with the new types of terms which appear in the case of a long-range potential. Our context here is particular since we consider potentials that satisfy the following assumptions:

Assumption 4.1. $\nabla \Phi$ is a Lipschitz function with fast decay such that

$$
\log (\log |\log \nabla \Phi(x)|) \geq \lambda\left(1+|x|^{2(d-1)}\right)
$$

with $\lambda$ a constant to be chosen later. 
Moreover we have

$$
x\left(\Phi^{-1}\left(\frac{x}{4}\right)\right)^{2} \underset{x \rightarrow \infty}{\longrightarrow}+\infty
$$

and

$$
\left|\Phi^{\prime} \circ \Phi\right| \geq I d \text {. }
$$

Remark 4.1. The main restriction in our context is the decay of $\nabla \Phi$. The two last hypotheses are technical and should not be difficult to obtain for a class of functions satisfying the imposed decay.

Let us recall the expression of $r^{P o t, a}$ and $r^{P o t, b}$ :

$$
\begin{aligned}
& r_{s, m+1}^{P o t, a}\left(0, t, Z_{s}\right):=\sum_{n=0}^{m} \int_{0}^{t-n \delta} Q_{s, s+n}\left(t-t_{n+1}\right) \\
& \frac{1}{\varepsilon} \sum_{\substack{i, j=1 \\
i \neq j}}^{s+n}\left[\nabla \Phi^{>}\left(\frac{x_{i}-x_{j}}{\varepsilon}\right) \cdot \nabla_{v_{i}} \tilde{f}_{N, R}^{(s+n)}\right]\left(t_{n+1}, Z_{s}\right) d t_{n+1}
\end{aligned}
$$

and

$$
\begin{aligned}
& r_{s, m+1}^{P o t, b}\left(0, t, Z_{s}\right):= \sum_{n=0}^{m} \int_{0}^{t-n \delta} Q_{s, s+n}\left(t-t_{n+1}\right) \frac{(N-(s+n))}{\varepsilon} \\
& \sum_{i=1}^{s+n}\left[\int_{\mathbf{T}^{d(N-(s+n))} \times \mathbf{R}^{d(N-(s+n))}} \nabla \Phi\left(\frac{x_{i}-x_{s+n+1}}{\varepsilon}\right) \cdot \nabla_{v_{i}} f_{N}\right. \\
&\left.\prod_{\substack{1 \leq l \leq s+n \\
s+n+1 \leq k \leq N}} \mathbf{1}_{\left\{\left|x_{l}-x_{k}\right|>R \varepsilon\right\}} d Z_{(s+n, N)}\right]\left(t_{n+1}, Z_{s}\right) d t_{n+1},
\end{aligned}
$$

On the previous papers, the classical strategy to deal wih the remainders was to use continuity estimates on the collision operator together with some a priori estimates on the marginals.

Let us define $X_{\varepsilon, k, \alpha}$ the space of continous functions $f_{k}$ defined on $\mathbf{T}^{d k} \times \mathbf{R}^{d k}$ such that

$$
\left\|f_{k}\right\|_{\varepsilon, k, \alpha}:=\sup _{Z_{k} \in \mathbf{T}^{d k} \times \mathbf{R}^{d k}}\left|f_{k}\left(Z_{k}\right) \exp \left(\alpha H_{k}^{<}\left(Z_{k}\right)\right)\right|<\infty
$$

where we recall

$$
H_{k}^{<}\left(Z_{k}\right):=\sum_{1 \leq i \leq k} \frac{1}{2}\left|v_{i}\right|^{2}+\sum_{1 \leq i<j \leq k} \Phi^{<}\left(\frac{x_{i}-x_{j}}{\varepsilon}\right) .
$$

In our context we can also obtain some a priori estimates on the truncated marginals applying the maximum principle for the Liouville equation.

Proposition 4.1. For any fixed $N$, considering the initial data (2.1.1) for any $s \geq 1$, we have the following uniform bound (with respect to time)

$$
\sup _{t \geq 0}\left\|\tilde{f}_{N, R}^{(s)}(t)\right\|_{\varepsilon, s, \beta} \leq\left\|\rho^{0}\right\|_{\infty}\left(\frac{\beta}{2 \pi}\right)^{d s / 2}\left(1-\varepsilon \kappa_{d} R^{d}\right)^{-s} \exp \left(\beta s^{2}\left\|\Phi^{>}\right\|_{\infty}\right) .
$$

The proof of Proposition 4.1 is quite classical and can be found in Appendix A. The problematic is then the following: thanks to our a priori estimates, we have a control on the marginals but not on the derivatives of the marginals. So the classical strategy can not work. The idea is to adopt a weak approach in order to make the derivatives act on a test function $\varphi: \mathbf{T}^{d} \times \mathbf{R}^{d} \rightarrow \mathbf{R}$. 


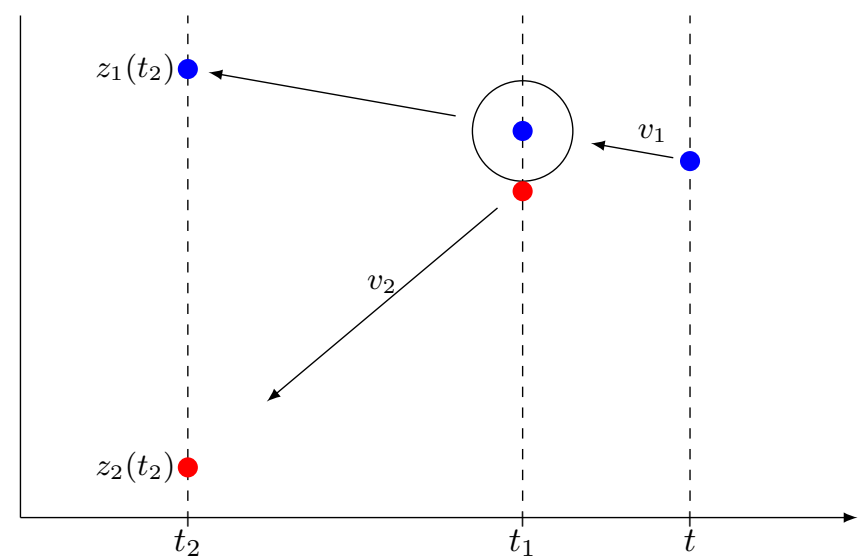

Figure 2. Representation of a pseudo-trajectory undergoing one collision in the pre-collisional case. By construction, at time $t_{1}$, we have $z_{1}\left(t_{1}\right)=\left(x_{1}-v_{1}\left(t-t_{1}\right), v_{1}\right)$ and $z_{2}\left(t_{1}\right)=\left(x_{1}-v_{1}\left(t-t_{1}\right)+\varepsilon \nu_{2}, v_{2}\right)$.

The advantage of the iteration method adopted is that, at this point, we know that there is no recollision in the pseudo-trajectories involved. Then, we can easily pass from the state of particles at $t_{m}$ to the state of particle 1 at $t$ via changes of variables defined as follows:

$$
\begin{aligned}
\rho_{m}: \mathbf{T}^{d} \times[0, t-\delta] \times \mathbf{S}^{d-1} \times \mathbf{R}^{d} \times \cdots \times\left[0, t_{m-1}-\delta\right] \times \mathbf{S}^{d-1} \times \mathbf{R}^{d} & \rightarrow \mathbf{T}^{(m+1) d} \times \mathbf{R}^{(m+1) d} \\
\left(z, t_{1}, \nu_{2}, v_{2}, \ldots, t_{m}, \nu_{m+1}, v_{m+1}\right) & \mapsto \tilde{Z}_{m+1}=Z_{m+1}\left(t_{m}\right)
\end{aligned}
$$

where $Z_{m+1}\left(t_{m}\right)$ is a pseudo-trajectory associated to a collision tree at time $t_{m}$. Then, the state of the particle 1 at time $t$ can be expressed in function of $\left(\tilde{x}_{1}, \tilde{v}_{1}, \ldots, \tilde{x}_{m+1}, \tilde{v}_{m+1}\right)$, the positions at time $t_{m}$.

Remark 4.2. We point out that there is as much changes of variables as pseudo-trajectories and so as much as collision trees.

\subsection{Lipschitz control of the pseudo-trajectory}

In this section, we establish that the functions which allow to pass from the state of particle 1 at time $t$ to states of particles at other times are Lipschitz. In order to do so, we will have to study the microscopic interaction.

Let us start with the following lemma about the microscopic time of interaction that we denote $\tau^{*}$.

Proposition 4.2. When the relative velocity has a lower bound equal to $\eta$, we have the following bound:

$$
\tau^{*} \leq C \frac{R^{2}}{\eta}
$$

with $C \geq 0$ a constant.

Proof. We consider two particles $z_{1}$ and $z_{2}$ which are precollisional at time $t_{-}$and study the following rescaled problem

$$
\left\{\begin{array}{l}
\frac{d y_{1}}{d \tau}=w_{1}, \quad \frac{d y_{2}}{d \tau}=w_{2} \\
\frac{d w_{1}}{d \tau}=-\nabla \Phi^{<}\left(y_{1}-y_{2}\right)=-\frac{d w_{2}}{d \tau} .
\end{array}\right.
$$

where

$$
\tau:=\frac{\left(t-t_{-}\right)}{\varepsilon}, \quad y(\tau):=\frac{x(\tau)}{\varepsilon}, \quad w(\tau):=v(\tau) .
$$


We recall here the expression of the microscopic time of interation $\tau^{*}$ obtain after a careful study of the dynamics (see [10] for more details)

$$
\tau^{*}:=2 \int_{\rho^{*}}^{R}\left(\mathcal{E}_{0}-\Psi\left(\rho, \mathcal{E}_{0}, \mathcal{I}_{0}\right)\right)^{-1 / 2} d \rho
$$

where $\mathcal{E}_{0}:=\left|\Delta w_{0}\right|^{2}, \mathcal{I}_{0}:=\frac{\left|\Delta y_{0} \wedge \Delta w_{0}\right|}{R \Delta w_{0}}=: \sin \alpha$ and $\Psi:=\frac{\mathcal{E}_{0} \mathcal{I}_{0}^{2} R^{2}}{\rho^{2}}+4 \Phi^{<}(\rho)$ and where $(\rho, w)$ are the polar coordinates of the trajectory and $\rho^{*}:=\max \left\{\rho_{0} \in(0, R) \mid \Psi\left(\rho, \mathcal{E}_{0}, \mathcal{I}_{0}\right)=\mathcal{E}_{0}\right\}$ is the minimal radius. $\Delta y_{0}$ and $\Delta w_{0}$ stand respectfully for the initial difference of rescaled positions and the initial difference of rescaled velocities.

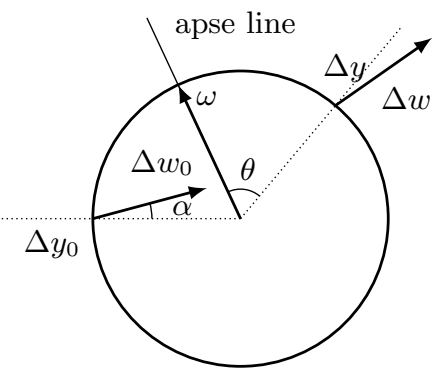

Figure 3. Representation of the reduced dynamics.

For the rest of the proof, we will drop the exponent "<" in $\nabla \Phi^{<}$for more clarity.

After the change of variables $y=\mathcal{E}_{0}-\Psi\left(\rho, \mathcal{E}_{0}, \mathcal{I}_{0}\right)$, using

$$
\frac{d}{d \rho}\left(\mathcal{E}_{0}-\Psi\left(\rho, \mathcal{E}_{0}, \mathcal{I}_{0}\right)\right)=\frac{2 \mathcal{E}_{0} \mathcal{I}_{0}^{2} R^{2}}{\rho^{3}}-4 \Phi^{\prime}(\rho) \geq \frac{2 \mathcal{E}_{0} \mathcal{I}_{0}^{2} R^{2}}{\rho^{3}} \geq \frac{2 \mathcal{E}_{0} \mathcal{I}_{0}^{2}}{R}
$$

we get the following bound

$$
\tau^{*} \leq \frac{R}{2 \mathcal{E}_{0} \mathcal{I}_{0}^{2}} 2 \int_{0}^{\mathcal{E}_{0}\left(1-\mathcal{I}_{0}^{2}\right)} \frac{1}{\sqrt{y}} d y \leq \frac{R}{\mathcal{E}_{0} \mathcal{I}_{0}^{2}} 2 \sqrt{\mathcal{E}_{0}\left(1-\mathcal{I}_{0}^{2}\right)}=\frac{2 R}{\sqrt{\mathcal{E}_{0}}} \frac{\sqrt{1-\mathcal{I}_{0}^{2}}}{\mathcal{I}_{0}^{2}} .
$$

Moreover, noticing that $\Phi\left(\rho^{*}\right) \leq \frac{\mathcal{E}_{0}}{4}$, then $\rho^{*} \geq \Phi^{-1}\left(\frac{\mathcal{E}_{0}}{4}\right)$ and we define $i_{0} \in(0,1)$ by $i_{0}:=$ $\frac{1}{2 \sqrt{2} R} \Phi^{-1}\left(\frac{\mathcal{E}_{0}}{4}\right)$. Then we distinguish two cases which are respectively associated to the two different following situations:

- the particle barely get into the sphere during the collision, arriving tangentially (or almost),

- the collision is head-on, which implies that the particle will deeply get into the sphere (but not for long).

Let us deal with these two situations.

(1) We start with the first one. Then we assume that $\mathcal{I}_{0} \geq i_{0}$. Therefore, we have

$$
\tau^{*} \leq \frac{2 R}{\sqrt{\mathcal{E}_{0} i_{0}^{2}}} \leq \frac{16 R^{2}}{\sqrt{\mathcal{E}_{0}}\left(\Phi^{-1}\left(\frac{\mathcal{E}_{0}}{4}\right)\right)^{2}} .
$$

By the assumption (4.0.8) on the potential, we have $\frac{1}{\sqrt{\mathcal{E}_{0}}\left(\Phi^{-1}\left(\frac{\mathcal{E}_{0}}{4}\right)\right)^{2}} \underset{\mathcal{E}_{0} \rightarrow \infty}{\longrightarrow} 0$ and $\Phi^{-1}\left(\frac{\mathcal{E}_{0}}{4}\right)$ $\underset{\mathcal{E}_{0} \rightarrow 0}{\longrightarrow} \infty$. Thus, there exists two constant $A_{1}, A_{2}>0$ such that for all $\mathcal{E}_{0}>A_{2}$

$$
\frac{1}{\sqrt{\mathcal{E}_{0}}\left(\Phi^{-1}\left(\frac{\mathcal{E}_{0}}{4}\right)\right)^{2}}<1<\frac{1}{\eta}
$$


for all $\mathcal{E}_{0}<A_{1}$,

$$
\frac{1}{\sqrt{\mathcal{E}_{0}}\left(\Phi^{-1}\left(\frac{\mathcal{E}_{0}}{4}\right)\right)^{2}}<\frac{1}{\sqrt{\mathcal{E}_{0}}}<\frac{1}{\eta}
$$

for $\eta^{2}<\mathcal{E}_{0}$. Moreover, $\mathcal{E}_{0} \mapsto \frac{1}{\sqrt{\mathcal{E}_{0}}\left(\Phi^{-1}\left(\frac{\mathcal{E}_{0}}{4}\right)\right)^{2}}$ is a continuous function on $\left[A_{1}, A_{2}\right]$ and is bounded and for $\mathcal{E}_{0} \in\left[A_{1}, A_{2}\right]$. Thus, for all $\mathcal{E}_{0}$ such that $\eta^{2}<\mathcal{E}_{0}$,

$$
\tau^{*} \leq C \frac{R^{2}}{\eta}
$$

where $C$ is a constant which does not depend on $R$ and $\eta$.

(2) Let us now assume $\mathcal{I}_{0} \leq i_{0}$ to deal with the second situation. We define $\gamma:=\Phi^{-1}\left(\frac{\mathcal{E}_{0}}{8}\right)$ and we split $\tau^{*}$ into two integrals

$$
\tau^{*}=\tau_{1}^{*}+\tau_{2}^{*}
$$

with $\tau_{1}^{*}:=2 \int_{\rho^{*}}^{\gamma}\left(\mathcal{E}_{0}-\Psi\left(\rho, \mathcal{E}_{0}, \mathcal{I}_{0}\right)\right)^{-1 / 2} d \rho$. Indeed, we have the following bound

$$
\frac{\mathcal{E}_{0} \mathcal{I}_{0}^{2}}{4\left(\rho^{*}\right)^{2}} R^{2} \leq \frac{\mathcal{E}_{0} i_{0}^{2}}{4\left(\rho^{*}\right)^{2}} R^{2} \leq \frac{\mathcal{E}_{0} i_{0}^{2}}{4 \times 8 i_{0}^{2}}=\frac{\mathcal{E}_{0}}{32} .
$$

Thus, we get that

$$
\frac{\mathcal{E}_{0}}{4}-\frac{\mathcal{E}_{0} \mathcal{I}_{0}^{2}}{4\left(\rho^{*}\right)^{2}} R^{2} \geq 7 \frac{\mathcal{E}_{0}}{32} \geq \frac{\mathcal{E}_{0}}{8}
$$

and then

$$
\rho^{*}=\Phi^{-1}\left(\frac{\mathcal{E}_{0}}{4}-\frac{\mathcal{E}_{0} \mathcal{I}_{0}^{2}}{4\left(\rho^{*}\right)^{2}} R^{2}\right) \leq \Phi^{-1}\left(\frac{\mathcal{E}_{0}}{8}\right)=\gamma
$$

We put $M(\Phi):=\inf _{\rho^{*} \leq \rho \leq \gamma}\left|\Phi^{\prime}(\rho)\right|>0$. We notice that because of assumption (4.0.9) on the potential, we have on $\left[\rho^{*}, \gamma\right]$

$$
\begin{aligned}
\frac{d}{d \rho}\left(\mathcal{E}_{0}-\Psi\right)=\frac{2 \mathcal{E}_{0} \mathcal{I}_{0}^{2} R^{2}}{\rho^{3}}-4 \Phi^{\prime}(\rho) & \geq 4 M(\Phi) \\
& \geq 4 \Phi^{\prime}\left(\Phi^{-1}\left(\frac{\mathcal{E}_{0}}{8}\right)\right) \\
& \geq \frac{\mathcal{E}_{0}}{2} .
\end{aligned}
$$

Finally, we have

$$
\begin{aligned}
\tau_{1}^{*} & \leq \frac{1}{\mathcal{E}_{0} / 2} 2 \int_{0}^{\mathcal{E}_{0} / 2-\mathcal{E}_{0} \mathcal{I}_{0}^{2} R^{2} / \gamma^{2}} \frac{d y}{\sqrt{y}} \\
& =\frac{\sqrt{\mathcal{E}_{0} / 2-\mathcal{E}_{0} \mathcal{I}_{0}^{2} R^{2} / \gamma^{2}}}{\mathcal{E}_{0} / 2} \leq \frac{\sqrt{2}}{\sqrt{\mathcal{E}_{0}}} \leq C \frac{R^{2}}{\eta} .
\end{aligned}
$$

Moreover, directly bounding the integrand in $\tau_{2}^{*}$, we get

$$
\tau_{2}^{*} \leq \frac{2 R}{\sqrt{\mathcal{E}_{0} / 2-\mathcal{E}_{0} \mathcal{I}_{0}^{2} R^{2} / \gamma^{2}}} .
$$

Using the fact that $\frac{\mathcal{E}_{0} \mathcal{I}_{0}^{2} R^{2}}{\gamma^{2}} \leq \frac{\mathcal{E}_{0} i_{0}^{2} R^{2}}{\gamma^{2}} \leq \frac{\mathcal{E}_{0}}{8}$, we get

$$
\tau_{2}^{*} \leq \frac{2 R}{\sqrt{\mathcal{E}_{0} / 2-\mathcal{E}_{0} / 8}}=\frac{4 \sqrt{2}}{\sqrt{3 \mathcal{E}_{0}}} R \leq C \frac{R^{2}}{\eta}
$$

which completes the proof. 


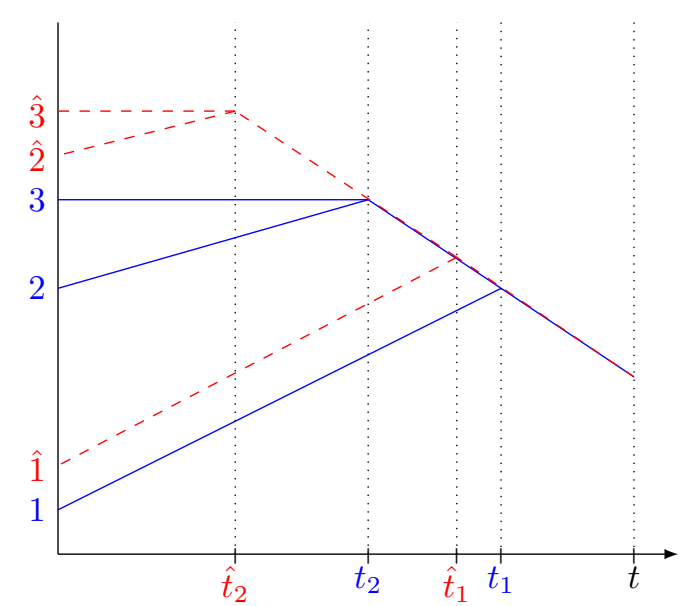

Figure 4. Representation of two collision trees with different times of collisions.

Let us go back to our study. In the following, we first deal with the particular case corresponding to observing two different trees with identical times of collisions. We will explain later how to deduce from it the general case with different times of collision for each tree as represented in Figure 4.

Before stating our result, let us prove the following lemma dealing with the case of two particles interacting.

Lemma 4.1. We denote by $\left(x_{1}^{0}, v_{1}^{0}\right),\left(x_{2}^{0}, v_{2}^{0}\right)$ and $\left(\hat{x}_{1}^{0}, \hat{v}_{1}^{0}\right),\left(\hat{x}_{2}^{0}, \hat{v}_{2}^{0}\right)$ two different initial positions associated to particles 1 and 2 , and by $\left(x_{1}, v_{1}\right),\left(x_{2}, v_{2}\right)$ and $\left(\hat{x}_{1}, \hat{v}_{1}\right),\left(\hat{x}_{2}, \hat{v}_{2}\right)$ their respective positions after scattering. Then, we have the following inequality:

$$
\left|x_{1}-\hat{x}_{1}\right|+\left|x_{2}-\hat{x}_{2}\right|+\left|v_{1}-\hat{v}_{1}\right|+\left|v_{2}-\hat{v}_{2}\right| \leq \frac{e^{C^{\prime} R^{2} / \eta}}{\varepsilon}\left(\left|x_{1}^{0}-\hat{x}_{1}^{0}\right|+\left|x_{2}^{0}-\hat{x}_{2}^{0}\right|+\left|v_{1}^{0}-\hat{v}_{1}^{0}\right|+\left|v_{2}-\hat{v}_{2}^{0}\right|\right)
$$

with $C^{\prime}$ a constant which depend on $\nabla \Phi$.

Proof. Let us go back to the study of the following dynamics

$$
\left\{\begin{array}{l}
\frac{d y_{1}}{d \tau}=w_{1}, \quad \frac{d y_{2}}{d \tau}=w_{2} \\
\frac{d w_{1}}{d \tau}=-\nabla \Phi^{<}\left(y_{1}-y_{2}\right)=-\frac{d w_{2}}{d \tau}
\end{array}\right.
$$

By hypothesis on $\nabla \Phi^{<}, \hat{\theta}:\left(\begin{array}{c}y_{1} \\ y_{2} \\ w_{1} \\ w_{2}\end{array}\right) \mapsto\left(\begin{array}{c}w_{1} \\ w_{2} \\ -\nabla \Phi^{<}\left(y_{1}-y_{2}\right) \\ \nabla \Phi^{<}\left(y_{1}-y_{2}\right)\end{array}\right)$ is $C^{\prime}$-Lipschitz where $C^{\prime}=\max \left(1, C_{\nabla \Phi}\right)$ with $C_{\nabla \Phi}$ the Lipschitz constant of $\nabla \Phi$. So by the Cauchy-Lipschitz theorem, given $U:=$ $\left(y_{1}, y_{2}, w_{1}, w_{2}\right)$ and $\hat{U}:=\left(\hat{y}_{1}, \hat{y}_{2}, \hat{w}_{1}, \hat{w}_{2}\right)$ with respective initial data $U^{0}:=\left(y_{1}^{0}, y_{2}^{0}, w_{1}^{0}, w_{2}^{0}\right)$ and $\hat{U}^{0}:=\left(\hat{y}_{1}^{0}, \hat{y}_{2}^{0}, \hat{w}_{1}^{0}, \hat{w}_{2}^{0}\right)$, then

$$
|U(\tau)-\hat{U}(\tau)| \leq e^{C^{\prime} \tau}\left|U^{0}-\hat{U}^{0}\right|
$$

and so

$\left|y_{1}-\hat{y}_{1}\right|+\left|y_{2}-\hat{y}_{2}\right|+\left|w_{1}-\hat{w}_{1}\right|+\left|w_{2}-\hat{w}_{2}\right| \leq e^{C^{\prime} \tau_{*}}\left(\left|y_{1}^{0}-\hat{y}_{1}^{0}\right|+\left|y_{2}^{0}-\hat{y}_{2}^{0}\right|+\left|w_{1}^{0}-\hat{w}_{1}^{0}\right|+\left|w_{2}^{0}-\hat{w}_{2}^{0}\right|\right)$ 
and finally

$\left|\frac{x_{1}}{\varepsilon}-\frac{\hat{x}_{1}}{\varepsilon}\right|+\left|\frac{x_{2}}{\varepsilon}-\frac{\hat{x}_{2}}{\varepsilon}\right|+\left|v_{1}-\hat{v}_{1}\right|+\left|v_{2}-\hat{v}_{2}\right| \leq e^{C^{\prime} \tau_{*}}\left(\left|\frac{x_{1}^{0}}{\varepsilon}-\frac{\hat{x}_{1}^{0}}{\varepsilon}\right|+\left|\frac{x_{2}^{0}}{\varepsilon}-\frac{\hat{x}_{2}^{0}}{\varepsilon}\right|+\left|v_{1}^{0}-\hat{v}_{1}^{0}\right|+\left|v_{2}^{0}-\hat{v}_{2}^{0}\right|\right)$.

So using $1 \leq \frac{1}{\varepsilon}$ on both sides, we finally obtain

$$
\begin{aligned}
\left|x_{1}-\hat{x}_{1}\right|+\left|x_{2}-\hat{x}_{2}\right|+\left|v_{1}-\hat{v}_{1}\right|+\left|v_{2}-\hat{v}_{2}\right| & \leq \frac{e^{C^{\prime} \tau_{*}}}{\varepsilon}\left(\left|x_{1}^{0}-\hat{x}_{1}^{0}\right|+\left|x_{2}^{0}-\hat{x}_{2}^{0}\right|+\left|v_{1}^{0}-\hat{v}_{1}^{0}\right|+\left|v_{2}^{0}-\hat{v}_{2}^{0}\right|\right) \\
& \leq \frac{e^{C^{\prime} R^{2} / \eta}}{\varepsilon}\left(\left|x_{1}^{0}-\hat{x}_{1}^{0}\right|+\left|x_{2}^{0}-\hat{x}_{2}^{0}\right|+\left|v_{1}^{0}-\hat{v}_{1}^{0}\right|+\left|v_{2}^{0}-\hat{v}_{2}^{0}\right|\right) .
\end{aligned}
$$

Using this result, we can now go back to the case of several collisions.

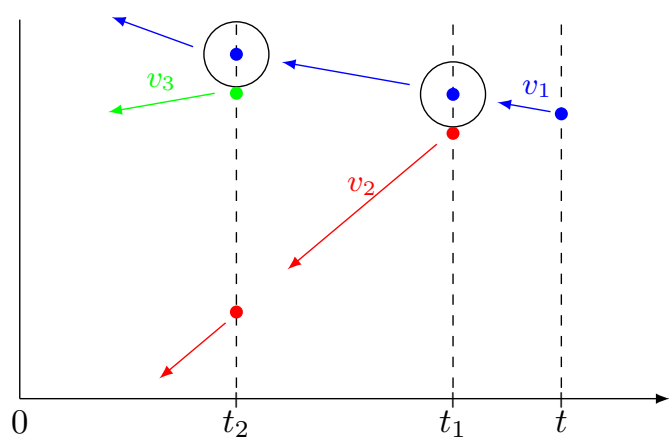

Figure 5. Representation of a pseudo-trajectory undergoing two collisions.

We have the following result:

Lemma 4.2. With the assumption of identical times of collisions for two different trees, the function $\left(\tilde{x}_{1}, \tilde{v}_{1}, \ldots, \tilde{x}_{k+1}, \tilde{v}_{k+1}\right) \mapsto z=z\left(\tilde{x}_{1}, \tilde{v}_{1}, \ldots, \tilde{x}_{k+1}, \tilde{v}_{k+1}\right)$, where $\left(\tilde{x}_{1}, \tilde{v}_{1}, \ldots, \tilde{x}_{k+1}, \tilde{v}_{k+1}\right)$ is the state of the $k+1$ particles after $k$ collisions at time $t_{k+1}^{+}$(i.e. before the $k+1$-th collision) on a pseudo-trajectory is a $\left(\tilde{C}_{R, \eta, \varepsilon}\right)^{k}$-Lipschitz function with $\tilde{C}_{R, \eta, \varepsilon}=\frac{C e^{C R^{2} / \eta}}{\varepsilon}$ and $C$ is a constant which can only depend on $\nabla \Phi$.

Proof. Let us prove this result by induction.

Initialization: We start with the post-collisional case. By construction of the pseudo-trajectory, particle 1 moves freely on $\left[t_{1}, t\right]$ and starts its scattering at $t_{1}$. Then, we deduce from Lemma 4.1 that

$$
\left|z_{1}\left(t_{1}\right)-\hat{z}_{1}\left(t_{1}\right)\right| \leq \frac{e^{C^{\prime} R^{2} / \eta}}{\varepsilon}\left[\left|z_{1}\left(t_{1}-t_{\varepsilon}\right)-\hat{z}_{1}\left(t_{1}-t_{\varepsilon}\right)\right|+\left|z_{2}\left(t_{1}-t_{\varepsilon}\right)-\hat{z}_{2}\left(t_{1}-t_{\varepsilon}\right)\right|\right]
$$

where $t_{\varepsilon}$ is the scattering time. Moreover, since by construction we know that particle 1 and 2 move freely on $\left[t_{2}^{+}, t_{1}-t_{\varepsilon}\right]$, we have

$$
\left|z_{1}\left(t_{1}-t_{\varepsilon}\right)-\hat{z}_{1}\left(t_{1}-t_{\varepsilon}\right)\right|+\left|z_{2}\left(t_{1}-t_{\varepsilon}\right)-\hat{z}_{2}\left(t_{1}-t_{\varepsilon}\right)\right| \leq C\left[\left|z_{1}\left(t_{2}^{+}\right)-\hat{z}_{1}\left(t_{2}^{+}\right)\right|+\left|z_{2}\left(t_{2}^{+}\right)-\hat{z}_{2}\left(t_{2}^{+}\right)\right|\right] .
$$

Thus, we have

$$
\left|z_{1}(t)-\hat{z}_{1}(t)\right| \leq \frac{C e^{C^{\prime} R^{2} / \eta}}{\varepsilon}\left[\left|z_{1}\left(t_{2}^{+}\right)-\hat{z}_{1}\left(t_{2}^{+}\right)\right|+\left|z_{2}\left(t_{2}^{+}\right)-\hat{z}_{2}\left(t_{2}^{+}\right)\right|\right]
$$


since particle 1 moves freely on $\left[t_{1}, t\right]$. Furthermore, since in the pre-collisional case, there is only free transport, the above inequality holds true for $\varepsilon$ small enough.

Induction step: By assumption on the inductive step, we have

$$
\begin{array}{r}
\left|z_{1}(t)-\hat{z}_{1}(t)\right| \leq\left(\frac{C e^{C R^{2} / \eta}}{\varepsilon}\right)^{k-1}\left(\left|z_{1}\left(t_{k}^{+}\right)-\hat{z}_{1}\left(t_{k}^{+}\right)\right|+\left|z_{2}\left(t_{k}^{+}\right)-\hat{z}_{2}\left(t_{k}^{+}\right)\right|+\ldots\right. \\
\left.\cdots+\left|z_{k}\left(t_{k}^{+}\right)-\hat{z}_{k}\left(t_{k}^{+}\right)\right|\right) .
\end{array}
$$

Let us denote by $m_{k}$ the "progenitor" of particle $k+1$ (i.e. $m_{k}$ is the particle it is added next to). By the initialization applied for particles $m_{k}$ and $k+1$, we have that

$$
\left|z_{m_{k}}\left(t_{k}\right)-\hat{z}_{m_{k}}\left(t_{k}\right)\right| \leq\left(\frac{C e^{C R^{2} \eta}}{\varepsilon}\right)\left[\left|z_{m_{k}}\left(t_{k+1}^{+}\right)-\hat{z}_{m_{k}}\left(t_{k+1}^{+}\right)\right|+\left|z_{k+1}\left(t_{k+1}^{+}\right)-\hat{z}_{k+1}\left(t_{k+1}^{+}\right)\right|\right]
$$

Moreover, by construction of the pseudo-trajectories with our iteration strategy, we know that the other particles move via free transport on $\left.] t_{k+1}^{+}, t_{k}\right]$. Then, we deduce that for $\varepsilon$ small enough,

$$
\left|z_{1}(t)-\hat{z}_{1}(t)\right| \leq\left(\frac{C e^{C R^{2} / \eta}}{\varepsilon}\right)^{k}\left[\left|z_{1}\left(t_{k+1}^{+}\right)-\hat{z}_{1}\left(t_{k+1}^{+}\right)\right|+\cdots+\left|z_{k+1}\left(t_{k+1}^{+}\right)-\hat{z}_{k+1}\left(t_{k+1}^{+}\right)\right|\right]
$$

which completes the proof.

Let us go back to the general case. For two different trees, we will denote by $t_{k}$ and $\hat{t}_{k}$ the respective times of collisions. Noticing that the scattering does not depend on time, the general case is only different from the one above because of translations in the positions. Indeed, for instance particle 1 starts its scattering at $t_{1}$ while particle $\hat{1}$ keeps moving freely on $\left[\hat{t}_{1}, t_{1}\right]$ in the case $\hat{t}_{1}<t_{1}$. Then, it will imply an error on the positions directly bounded by $R\left|t_{1}-\hat{t}_{1}\right|$, the velocities being bounded by $R$. Thus, if we prove that collisions times are also Lipschitz, we will be able to conclude.

Before proving it, we need to make a precision not necessary until now. In our iteration strategy, when we truncate to remove recollisions (we will explain how exactly in Section 6.1), the geometrical set observed will be one from which we will also remove grazing collisions by truncating parameters such that the angle between $\nu^{s+1}$ and $\left(v_{s+1}-v_{i}\right)$ belongs to $[\pi / 2-\varepsilon, \pi / 2]$. The measure of the set associated to possible recollisions and grazing collisions being of the same order as the one of the set only associated to possible recollisions, the corresponding term will vanish exactly the same way as if we would have only remove recollisions.

Lemma 4.3. We denote by $t_{c}$ a time of collision and by $x$ and $v$ the respective relative positions and relative velocities of the two particles colliding with $\eta \leq|v|$. Then, we have

$$
\left\|\frac{\partial t_{c}}{\partial x^{i}}\right\|_{\infty}+\left\|\frac{\partial t_{c}}{\partial v^{i}}\right\|_{\infty} \leq \frac{C}{\eta\left|\cos \left(\frac{\pi}{2}-\varepsilon\right)\right|}
$$

with $C$ a constant and where $x^{i}$ and $v^{i}$ are the $i$-th coordinates of respectively $x$ and $v$.

Proof. Starting from the following equality

$$
\left|x-t_{c} v\right|=R \varepsilon
$$

which characterizes collision times, we differentiate it and obtain

$$
\frac{\partial t_{c}}{\partial x^{i}}=\frac{n^{i}}{n \cdot v} \quad \text { and } \quad \frac{\partial t_{c}}{\partial v^{i}}=\frac{t_{c} n^{i}}{n \cdot v},
$$

with $n$ the vector of norm 1 associated to the collision and $n^{i}$ its $i$-th coordinate. Therefore, using the bound $\eta \leq|v|$ and the construction of the iteration term regarding the grazing collisions leads to the conclusion. 
Finally, using the two above lemmas we can deduce the following result:

Corollary 4.3. The function $\left(\tilde{x}_{1}, \tilde{v}_{1}, \ldots, \tilde{x}_{k+1}, \tilde{v}_{k+1}\right) \mapsto z=z\left(\tilde{x}_{1}, \tilde{v}_{1}, \ldots, \tilde{x}_{k+1}, \tilde{v}_{k+1}\right)$, where $\left(\tilde{x}_{1}, \tilde{v}_{1}, \ldots\right.$, $\left.\tilde{x}_{k+1}, \tilde{v}_{k+1}\right)$ is the state of the $k+1$ particles after $k$ collisions at time $t_{k+1}^{+}$(i.e. before the $k+1$-th collision) on a pseudo-trajectory is a $\left(C_{R, \eta, \varepsilon}\right)^{k}$-Lipschitz function with $C_{R, \eta, \varepsilon}=\frac{C R e^{C R^{2} / \eta}}{\eta\left|\cos \left(\frac{\pi}{2}-\varepsilon\right)\right| \varepsilon}$ and $C$ is a constant which can only depend on $\nabla \Phi$.

\subsection{Estimates of the remainders associated to the long-range part of the potential}

From now on, $C$ is a constant which can depend on $\varphi, \rho^{0}, \beta$ and which can include factors as $\left(1-\varepsilon \kappa_{d} R^{d}\right)^{-1}$ or again $\exp \left(\beta\left\|\Phi^{>}\right\|_{\infty}\right)$. This is not a problem since those two last terms converges to 1 as $N$ goes to $\infty$. Moreover, we will assume that $\varepsilon<\eta$. Indeed, the orders of magnitude adopted in section 7 will satisfy this property.

First of all, let us notice that we can rewrite the remainder $r_{N}^{P o t, a}(t)$ as follows:

$$
\begin{aligned}
r_{N}^{P o t, a, K}(t) & :=\sum_{k=1}^{K} \sum_{j_{1}=0}^{n_{1}-1} \cdots \sum_{j_{k-1}=0}^{n_{k-1}-1} Q_{1, J_{1}}(\tau) \ldots Q_{J_{k-2}, J_{k-1}}(\tau) r_{J_{k-1}, n_{k}}^{P o t, a}(t-k \tau, t-(k-1) \tau) \\
& =\sum_{k=1}^{K} \sum_{j_{1}=0}^{n_{1}-1} \cdots \sum_{j_{k}=0}^{n_{k}-1} F^{P o t, a}\left(J^{(k)}, z\right)
\end{aligned}
$$

where for $J^{(k)}=\left(1, j_{1}, \ldots, j_{k}\right)$ fixed, we denote

$$
\begin{aligned}
F^{P o t, a}\left(J^{(k)}, z\right):= & Q_{1, J_{1}}(\tau) \ldots Q_{J_{k-2}, J_{k-1}}(\tau) \\
& \int_{0}^{\tau-j_{k} \delta} Q_{J_{k-1}, J_{k}}\left(\tau-t_{J_{k}}\right) \frac{1}{\varepsilon} \sum_{\substack{i, j=1 \\
i \neq j}}^{J_{k}}\left[\nabla \Phi^{>}\left(\frac{x_{i}-x_{j}}{\varepsilon}\right) \cdot \nabla_{v_{i}} \tilde{f}_{N, R}^{\left(J_{k}\right)}\right]\left(t_{J_{k}}, z\right) d t_{J_{k}} .
\end{aligned}
$$

Proposition 4.4. Let $k$ be an integer, $1 \leq k \leq K$. There exists a constant $C$ such that for any $\tau>0, J^{(k)}=\left(j_{1}, \ldots, j_{k}\right)$ with $j_{i} \in\left\{0, \ldots, n_{i}-1\right\}$ for all $i$,

$$
\left|\int_{\mathbf{T}^{d} \times \mathbf{R}^{d}} \varphi(z) F^{P o t, a}\left(J^{(k)}, z\right) d z\right| \leq C\left[\left(\frac{C R e^{C \frac{R^{2}}{\eta}}}{\eta\left|\cos \left(\frac{\pi}{2}-\varepsilon\right)\right| \varepsilon}\right)^{J_{k}}+\frac{J_{k}}{\varepsilon^{2}}\right]\left\|\nabla \Phi^{>}\right\|_{\infty}(k \tau)^{J_{k}}\left(R^{d-1} E^{d+1}\right)^{J_{k}} .
$$

Proof. For each collision tree $p$, we know that the changes of variables associated

$$
\rho^{p}:\left(z, t_{1}, \nu_{2}, v_{2}, \ldots, t_{J_{k}-1}, \nu_{J_{k}}, v_{J_{k}}\right) \mapsto Z_{J_{k}}\left(t_{J_{k}}\right),
$$

where $Z_{J_{k}}\left(t_{J_{k}}\right)$ is the pseudo-trajectory at time $t_{J_{k}}$ associated to the tree $p$, maps the measure

$$
\left.\prod_{i=1}^{J_{k}-1}(R \varepsilon)^{d-1}\left(v_{i+1}-v_{m_{i}}\left(t_{i}\right)\right) \cdot \nu_{i+1}\right) d z d t_{1} \ldots d t_{J_{k}-1} d \nu_{2} \ldots d \nu_{J_{k}} d v_{1} \ldots d v_{J_{k}}
$$


on the Lebesgue measure $d Z_{J_{k}}$. So applying those ones, we get that

$$
\begin{aligned}
& \left|\int_{\mathbf{T}^{d} \times \mathbf{R}^{d}} \varphi(z) F^{P o t, a}\left(J^{(k)}, z\right) d z\right| \\
& \leq N^{J_{k}-1} \sum_{p} \mid \int_{0}^{k \tau-\left(J_{k}-1\right) \delta} \int_{\operatorname{Im}\left(\rho^{p}\right)} \chi \varphi\left(z\left(x_{1}, v_{1}, \ldots, x_{J_{k}}, v_{J_{k}}\right)\right) \\
& \frac{1}{\varepsilon} \sum_{\substack{i, j=1 \\
i \neq j}}^{J_{k}} \nabla \Phi^{>}\left(\frac{x_{i}-x_{j}}{\varepsilon}\right) \cdot \nabla_{v_{i}} \tilde{f}_{N, R}^{\left(J_{k}\right)}\left(t_{J_{k}}, Z_{J_{k}}\right) d Z_{J_{k}} d t_{J_{k}} \mid
\end{aligned}
$$

where we will denote by $\chi$ the product of all the "smooth indicator function" involved in the construction of those terms.

Moreover, since $\chi=\prod_{i=2}^{J_{k}}\left[\chi_{H_{i}} \chi_{\text {geom }(i)} \chi_{\eta_{i}}\right]$ and for each $i$,

$$
\left|\nabla\left[\chi_{H_{i}} \chi_{\text {geom }(i)} \chi_{\eta_{i}}\right]\right| \leq C\left(1+\frac{1}{\varepsilon}+\frac{1}{\eta}\right) \leq \frac{C}{\varepsilon},
$$

(see (3.1.2), (3.1.3), (3.1.4)) we have

$$
|\nabla \chi| \leq \frac{J_{k}}{\varepsilon}
$$

In addition, applying Corollary 4.3 we have

$$
\begin{aligned}
\left|\nabla_{v_{i}} \varphi\left(z\left(x_{1}, v_{1}, \ldots, x_{J_{k}}, v_{J_{k}}\right)\right)\right| & \leq\|\nabla \varphi\|_{\infty}\left|\nabla_{v_{i}} z\left(x_{1}, v_{1}, \ldots, x_{J_{k}}, v_{J_{k}}\right)\right| \\
& \leq\|\nabla \varphi\|_{\infty}\left(\frac{C R e^{C R^{2} / \eta}}{\eta\left|\cos \left(\frac{\pi}{2}-\varepsilon\right)\right| \varepsilon}\right)^{J_{k}-1} .
\end{aligned}
$$

Noticing that integrating the measure (4.2.5) on the domain of integration that we consider gives a constant which is $O\left(\left(\prod_{i=1}^{J_{k}-1}(R \varepsilon)^{d-1} E\right) E^{d J_{k}} \frac{(k \tau)^{J_{k}}}{J_{k} !}\right)$, then after doing an integration by parts on the right-hand side of inequality (4.2.6), we deduce from (4.2.7) and (4.2.8) that

$$
\begin{aligned}
& \left|\int_{\mathbf{T}^{d} \times \mathbf{R}^{d}} \varphi(z) F^{\text {Pot,a }}\left(J^{(k)}, z\right) d z\right| \\
& \leq N^{J_{k}-1}\left(J_{k}\right) !\left[\left(\frac{C R e^{C R^{2} / \eta}}{\eta\left|\cos \left(\frac{\pi}{2}-\varepsilon\right)\right| \varepsilon}\right)^{J_{k}-1}+\frac{J_{k}}{\varepsilon}\right] \frac{1}{\varepsilon}\left\|\nabla \Phi^{>}\right\|_{\infty} \sup _{t \geq 0}\left\|\tilde{f}_{N, R}^{\left(J_{k}\right)}(t)\right\|_{\infty} \\
& \leq C\left[\left(\frac{\left.C R)^{d-1}\right)^{J_{k}-1} E^{(d+1) J_{k}} \frac{(k \tau)^{J_{k}}}{J_{k} !}}{\eta\left|\cos \left(\frac{\pi}{2}-\varepsilon\right)\right| \varepsilon}\right)^{J_{k}}+\frac{J_{k}}{\varepsilon^{2}}\right]\left\|\nabla \Phi^{>}\right\|_{\infty}\left(R^{d-1} E^{d+1}\right)^{J_{k}}(k \tau)^{J_{k}} .
\end{aligned}
$$

Quite similarly, we can rewrite the remainder $r_{N}^{P o t, b}(t)$ as follows:

$$
r_{N}^{P o t, b, K}(t)=\sum_{k=1}^{K} \sum_{j_{1}=0}^{n_{1}-1} \cdots \sum_{j_{k}=0}^{n_{k}-1} F^{P o t, b}\left(J^{(k)}, z\right)
$$


where for $J^{(k)}=\left(1, j_{1}, \ldots, j_{k}\right)$ fixed, we denote

$$
\begin{gathered}
F^{P o t, b}\left(J^{(k)}, z\right):=Q_{1, J_{1}}(\tau) \ldots Q_{J_{k-2}, J_{k-1}}(\tau) \int_{0}^{\tau-j_{k} \delta} Q_{J_{k-1}, J_{k}}\left(\tau-t_{J_{k}}\right) \frac{\left(N-J_{k}\right)}{\varepsilon} \\
\sum_{i=1}^{J_{k}}\left[\int_{\mathbf{T}^{d\left(N-J_{k}\right)} \times \mathbf{R}^{d\left(N-J_{k}\right)}} \nabla \Phi\left(\frac{x_{i}-x_{J_{k}+1}}{\varepsilon}\right) \cdot \nabla_{v_{i}} f_{N} \prod_{\substack{1 \leq l \leq J_{k} \\
J_{k}+1 \leq j \leq N}} \mathbf{1}_{\left\{\left|x_{l}-x_{j}\right|>R \varepsilon\right\}} d Z_{\left(J_{k}+1, N\right)}\right]\left(t_{J_{k}}, z\right) d t_{J_{k}} .
\end{gathered}
$$

Using a quite similar reasoning and the fact that $N \varepsilon^{d-1}=1$, we get an equivalent proposition for $r^{P o t, b}$.

Proposition 4.5. Let $k$ be an integer, $1 \leq k \leq K$. There exists a constant $C$ such that for any $\tau>0, J=\left(j_{1}, \ldots, j_{k-1}\right)$ with $j_{i} \in\left\{0, \ldots, n_{i}-1\right\}$ for all $i$,

$$
\left|\int_{\mathbf{T}^{d} \times \mathbf{R}^{d}} \varphi(z) F^{P o t, b}\left(J^{(k)}, z\right) d z\right| \leq \frac{C}{\varepsilon^{d-1}}\left[\left(\frac{C R e^{C \frac{R^{2}}{\eta}}}{\eta\left|\cos \left(\frac{\pi}{2}-\varepsilon\right)\right| \varepsilon}\right)^{J_{k}}+\frac{J_{k}}{\varepsilon^{2}}\right]\left\|\nabla \Phi^{>}\right\|_{\infty}(k \tau)^{J_{k}}\left(R^{d-1} E^{d+1}\right)^{J_{k}} .
$$

\section{Continuity estimates and control of some remainders}

In this section, we will use the classical techniques based on some continuity estimates to control the remainders associated to superexponential growth and to clusters.

First of all, let us recall here the general result concerning continuity estimates for the collision operators. In order for the paper to be more self-contained, we recall the proof in Appendix C. We denote by $|Q|_{s, s+n}$ the operators obtained by summing the absolute values of all elementary contributions

$$
\begin{array}{r}
|Q|_{s, s+n}(t):=\int_{0}^{t-\delta} \int_{0}^{t_{1}-\delta} \ldots \int_{0}^{t_{n-1}-\delta} \mathcal{S}_{s}\left(t-t_{1}\right)\left|\mathcal{C}_{s, s+1}\right| \mathcal{S}_{s+1}\left(t_{1}-t_{2}\right)\left|\mathcal{C}_{s+1, s+2}\right| \ldots \\
\ldots \mathcal{S}_{s+n-1}\left(t_{n-1}-t_{n}\right)\left|\mathcal{C}_{s+n-1, s+n}\right| d t_{n} \ldots d t_{1} .
\end{array}
$$

where

$$
\begin{aligned}
& \left(\left|\mathcal{C}_{s, s+1}\right| f_{N}^{(s+1)}\right)\left(Z_{s}\right) \\
& :=(N-s)(R \varepsilon)^{d-1} \sum_{i=1}^{s} \int_{\mathbf{S}^{d-1} \times \mathbf{R}^{d}}\left(\prod_{\substack{j=1 \\
j \neq i}}^{s} \mathbf{1}_{\left\{\left|x_{j}-x_{s+1}>R \varepsilon\right|\right\}}\right)_{f_{N}^{(s+1)}\left(\ldots, x_{i}, v_{i}^{*}, \ldots, x_{i}+R \varepsilon \nu, v_{s+1}^{*}\right) d \nu d v_{s+1}}^{f_{N}}\left(\nu .\left(v_{s+1}-v_{i}\right)\right)_{+} \\
& +(N-s)(R \varepsilon)^{d-1} \sum_{i=1}^{s} \int_{\mathbf{S}^{d-1} \times \mathbf{R}^{d}}\left(\prod_{\substack{j=1 \\
j \neq i}}^{s} \mathbf{1}_{\left\{\left|x_{j}-x_{s+1}>R \varepsilon\right|\right\}}\right)_{f_{N}^{(s+1)}\left(\ldots,\left(v_{s+1}-v_{i}\right)\right)_{-}}^{\left(\ldots, v_{i}, \ldots, x_{i}+R \varepsilon \nu, v_{s+1}\right) d \nu d v_{s+1} .}
\end{aligned}
$$

We deliberately omit some "smooth indicator functions" involved for more concision but the result holds true in their presence. 
Lemma 5.1. There is a constant $C_{d}$ depending only on $d$ such that for all $s, n \in \mathbf{N}, t \geq 0$, the operators $|Q|_{s, s+n}(t)$ satisfy the following continuity estimates: for all $f_{s+n} \in X_{\varepsilon, s+n, \alpha},|Q|_{s, s+n}(t) f_{s+n}$ belongs to $X_{\varepsilon, s, \frac{\alpha}{2}}$ and

$$
\left\||Q|_{s, s+n}(t) f_{s+n}\right\|_{\varepsilon, s, \frac{\alpha}{2}} \leq e^{s-1}\left(\frac{C_{d} R^{d-1} t}{\alpha^{\frac{d+1}{2}}}\right)^{n}\left\|f_{s+n}\right\|_{\varepsilon, s+n, \alpha}
$$

\subsection{Remainders with a superexponential growth}

Proposition 5.1. Let $k$ be an integer, $1 \leq k \leq K$. There exists a constant $C$ such that for any $\tau>0, J=\left(j_{1}, \ldots, j_{k-1}\right)$ with $j_{i} \in\left\{0, \ldots, n_{i}-1\right\}$ for all $i$,

$$
\begin{aligned}
& \mid \int_{\mathbf{T}^{d} \times \mathbf{R}^{d}} \varphi(z) Q_{1, J_{1}}(\tau) Q_{J_{1}, J_{2}}(\tau) \ldots Q_{J_{k-2}, J_{k-1}}(\tau) r_{J_{k-1}, n_{k}}^{\text {Supexp }}(t-k \tau,-(k-1) \tau, z) d z \mid \\
& \leq C\left[\left(\tilde{C} R^{d-1}\right)^{2} t \tau\right]^{2^{k}}
\end{aligned}
$$

with $\tilde{C}:=\frac{C_{d}}{\sqrt{\beta}}\left(1-\varepsilon \kappa_{d} R^{d}\right)^{-1} \exp \left(\beta\|\Phi\|_{\infty}\right), r_{J_{k-1}, n_{k}}^{\text {Supexp }}(t-k \tau, t-(k-1) \tau, z):=Q_{J_{k-1}, J_{k-1}+n_{k}}(\tau) \tilde{f}_{N, R}^{\left(J_{k-1}+n_{k}\right)}$.

Proof. Obviously, we have

$$
\begin{array}{r}
\left|\int_{\mathbf{T}^{d} \times \mathbf{R}^{d}} \varphi(z) Q_{1, J_{1}}(\tau) Q_{J_{1}, J_{2}}(\tau) \ldots Q_{J_{k-2}, J_{k-1}}(\tau) r_{J_{k-1}, n_{k}}^{\text {Supexp }}(t-k \tau, t-(k-1) \tau, z) d z\right| \\
\leq C\|\varphi\|_{\infty}\left\||Q|_{1, J_{k-1}}((k-1) \tau) r_{J_{k-1}, n_{k}}^{\text {Supexp }}(t-k \tau, t-(k-1) \tau)\right\|_{L^{\infty}\left(\mathbf{T}^{d} \times \mathbf{R}^{d}\right)} .
\end{array}
$$

Indeed, the exact distribution of collisions in the last $k-1$ intervals is not needed. Then, applying Lemma C.1 a first time, we get

$$
\begin{aligned}
& \left\||Q|_{1, J_{k-1}}((k-1) \tau) r_{J_{k-1}, n_{k}}^{\text {Supexp }}(t-k \tau, t-(k-1) \tau)\right\|_{L^{\infty}\left(\mathbf{T}^{d} \times \mathbf{R}^{d}\right)} \\
& \quad \leq\left(\frac{R^{d-1} C_{d}(k-1) \tau}{\beta^{(d+1) / 2}}\right)^{J_{k-1}-1}\left\|r_{J_{k-1}, n_{k}}^{\text {Supexp }}(t-k \tau, t-(k-1) \tau)\right\|_{\varepsilon, J_{k-1}, \beta / 2} .
\end{aligned}
$$

Observing the expression of $r_{J_{k-1}, n_{k}}^{\text {Supexp }}(t-k \tau, t-(k-1) \tau)$, we apply Lemma C.1 a second time and we finally get

$$
\begin{aligned}
& \left\||Q|_{1, J_{k-1}}((k-1) \tau) r_{J_{k-1}, n_{k}}^{\text {Supexp }}(t-k \tau, t-(k-1) \tau)\right\|_{L^{\infty}\left(\mathbf{T}^{d} \times \mathbf{R}^{d}\right)} \\
& \quad \leq\left(\frac{R^{d-1} C_{d}(k-1) \tau}{\beta^{(d+1) / 2}}\right)^{J_{k-1}-1}\left(\frac{R^{d-1} C_{d} \tau}{\beta^{(d+1) / 2}}\right)^{n_{k}}\left\|\sup _{t \geq 0} \tilde{f}_{N, R}^{\left(J_{k-1}+n_{k}\right)}(t)\right\|_{\varepsilon, J_{k-1}+n_{k}, \beta} .
\end{aligned}
$$

Using the a priori estimate on the truncated marginals (4.0.14), the fact that $(k-1) \tau \leq t$ and that $J_{k-1} \leq 2^{k}=n_{k}$, we get the final result.

\subsection{Remainders with clusters}

As previously, we notice that we can rewrite the term $r_{N}^{C l u, K}(t)$ as follows

$$
r_{N}^{C l u, K}(t)=\sum_{k=1}^{K} \sum_{j_{1}=0}^{n_{1}-1} \cdots \sum_{j_{k}=0}^{n_{k}-1} F^{C l u}\left(J^{(k)}, z\right)
$$

where for $J^{(k)}=\left(1, j_{1}, \ldots, j_{k}\right)$ fixed, we denote

$$
F^{C l u}\left(J^{(k)}, z\right):=Q_{1, J_{1}}(\tau) \ldots Q_{J_{k-2}, J_{k-1}}(\tau) \int_{0}^{\tau-j_{k} \delta} Q_{J_{k-1}, J_{k}}\left(\tau-t_{J_{k}}\right) \mathcal{C}_{J_{k}, J_{k}+1} \bar{f}_{N, R}^{\left(J_{k}+1\right)}\left(t_{J_{k}}, z\right) d t_{J_{k}} .
$$


So using the continuity estimates of the collision operators, a control on the cluster part should be enough. Let us recall here its expression

$$
\begin{aligned}
\bar{f}_{N, R}^{(s+1)}\left(t, Z_{s+1}\right):=\int_{\mathbf{T}^{d(N-(s+1))} \times \mathbf{R}^{d(N-(s+1))}} & f_{N}\left(t, Z_{N}\right) \\
& \left(\prod_{\substack{1 \leq k \leq s \\
s+2 \leq l \leq N}} \mathbf{1}_{\left|x_{k}-x_{l}\right|>R \varepsilon}\right)\left(1-\prod_{j=s+2}^{N} \mathbf{1}_{\left|x_{j}-x_{s+1}\right|>R \varepsilon}\right) d Z_{(s+2, N)} .
\end{aligned}
$$

We are actually able to obtain some a priori estimates for it too using again the maximum principle for the Liouville equation. Let us state the result here, the proof could be found in Appendix A.

Proposition 5.2. For any fixed $N$, considering the initial data (2.1.1) for any $s \geq 1$, we have the following uniform bound (with respect to time)

$$
\sup _{t \geq 0}\left\|\bar{f}_{N, R}^{(s)}(t)\right\|_{\varepsilon, s, \beta} \leq\left\|\rho^{0}\right\|_{\infty}\left(\frac{\beta}{2 \pi}\right)^{d s / 2}\left(1-\varepsilon \kappa_{d} R^{d}\right)^{-(s-1)} \exp \left(\beta s^{2}\left\|\Phi^{>}\right\|_{\infty}\right) R^{d} \varepsilon .
$$

Finally, we get the following estimate for the remainder associated to the cluster part.

Proposition 5.3. Let $k$ be an integer, $1 \leq k \leq K$. There exists a constant $C$ such that for any $\tau>0, J=\left(j_{1}, \ldots, j_{k-1}\right)$ with $j_{i} \in\left\{0, \ldots, n_{i}-1\right\}$ for all $i$,

$$
\left|\int_{\mathbf{T}^{d} \times \mathbf{R}^{d}} \varphi(z) F^{C l u}\left(J^{(k)}, z\right) d z\right| \leq C R^{d} \varepsilon\left(\tilde{C} R^{d-1} t\right)^{J_{k}} .
$$

Proof. First of all, doing the exact same reasoning as in the proof of Proposition 5.1, we get the following bound

$$
\begin{aligned}
\left|\int_{\mathbf{T}^{d} \times \mathbf{R}^{d}} \varphi(z) F^{C l u}\left(J^{(k)}, z\right) d z\right| & \\
\leq C\|\varphi\|_{\infty}\left(\frac{R^{d-1} C_{d}(k-1) \tau}{\beta^{(d+1) / 2}}\right)^{J_{k-1}-1} & \\
& \quad\left\|\int_{0}^{\tau-\delta} Q_{J_{k-1}, J_{k}}\left(\tau-t_{J_{k}}\right) \mathcal{C}_{J_{k}, J_{k}+1} \bar{f}_{N, R}^{\left(J_{k}+1\right)}\left(t_{J_{k}}, z\right) d t_{J_{k}}\right\|_{\varepsilon, J_{k-1}, \beta / 2} .
\end{aligned}
$$

Using again the Lemma C.1, we get that

$$
\begin{aligned}
\| \int_{0}^{\tau-\delta} Q_{J_{k-1}, J_{k}}\left(\tau-t_{J_{k}}\right) \mathcal{C}_{J_{k}, J_{k}+1} \bar{f}_{N, R}^{\left(J_{k}+1\right)}\left(t_{J_{k}}, z\right) d t_{J_{k}} & \|_{\varepsilon, J_{k-1}, \beta / 2} \\
& \leq\left(\frac{R^{d-1} C_{d} \tau}{\beta^{(d+1) / 2}}\right)^{j_{k}+1} \sup _{t \geq 0}\left\|\bar{f}_{N, R}^{\left(J_{k}\right)}(t)\right\|_{\varepsilon, J_{k}, \beta} .
\end{aligned}
$$

So finally, using the control of the cluster part in the above inequality together with the fact that $(k-1) \tau \leq t$ leads to the conclusion.

\section{Remainders and geometrical control}

\subsection{Some geometrical considerations}

We recall that in order to get the convergence, our aim is to couple the pseudo-trajectories associated to the BBGKY series to the ones of the Boltzmann with cut-off. As we mentioned previously the main difference between those two terms comes from the possible recollisions that can occur in the BBGKY case while they never occur in the Boltzmann case. 


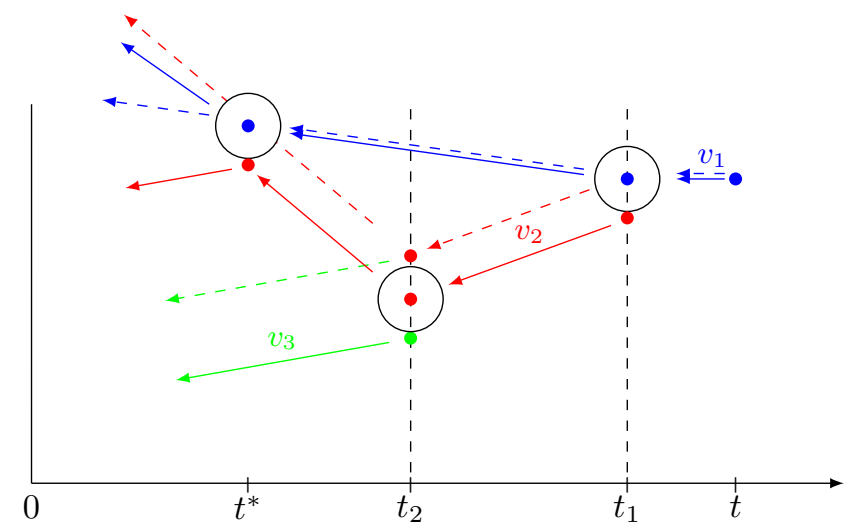

Figure 6. An example of a recollision between particles 1 and 2 at time $t^{*}$. As it can be observed the distance between the BBGKY trajectories and the Boltzmann ones (i.e. between the plain arrows and the dashed ones) is small until a recollision happens between particles 1 and 2 . Then the plain and the dashed arrows do not behave the same way at all and the distance between them increases linearly in time.

\subsubsection{Adjunction of one particle}

In the following, we just deal with the pre-collisional case. Let us explain why it is enough. What we do is slightly different from what has be done in the previous papers. Indeed, instead of working with the fact that some sets are small in the measure $d \nu_{k} d v_{k}$ for $v_{m_{k}}$ fixed to prevent recollisions, we use the idea that if we consider all the parameters and not only $\left(\nu_{k}, v_{k}\right)$, the scattering preserves the measure since it is an involution. In other words, instead of cutting only the last integral in $\left(\nu_{k}, v_{k}\right)$ with $v_{m_{k}}$ fixed, we cut all the integrals. Therefore, we will always define the pathological sets leading to possible recollisions in function of the pre-collisional parameters. The key point is that if some sets are small in those parameters, thanks to the property of the scattering mentioned above, they remain small in post-collisional parameters.

Let us go back to the study of the pre-collisional case. One of the advantages is that particles move freely in that case, making almost straightforward the study of possible recollisions. The strategy is to construct a set outside of which there will be no recollisions using the notion of good configurations and geometrical arguments. We recall that as mentioned in Definition 3.1 for $k$ particles the set of good configurations $\mathcal{G}_{k}\left(\varepsilon_{0}\right)$ is a set such that the particles remain at a distance $\varepsilon_{0}$. We fix the parameters with the following orders of magnitude $2^{K+1} R \varepsilon \ll \bar{a} \ll \varepsilon_{0} \ll \min (\delta E, 1)$ with $K$ some large integer, $\delta>0, E>0$ parameters to be chosen later. The set $\mathcal{G}_{k}\left(\varepsilon_{0}\right)$ has the obvious following properties:

Proposition 6.1. For particles in $\mathcal{G}_{k}\left(\varepsilon_{0}\right)$ the transport $\Psi_{k}$ coincides with the free flow. Moreover, if at time $t$ the configurations $Z_{k}, Z_{k}^{0}$ are such that

$$
\forall i \leq k,\left|x_{i}-x_{i}^{0}\right| \leq \bar{a}, v_{i}=v_{i}^{0}
$$

and that $Z_{k}^{0}$ belongs to $\mathcal{G}_{k}\left(\varepsilon_{0}\right)$, then the configurations $\Psi_{k}(u) Z_{k}, \Psi_{k}^{0}(u) Z_{k}^{0}$ will remain at a distance less than $\bar{a}$ for $u \in[0, t]$.

The main concern is to prove that the good configurations are stable by adjunction of the $k+1$-th particle by using the following lemma which state a control on free trajectories.

Lemma 6.1. Given $t>0$, and $\bar{a}>0$ satisfying $2^{K+1} R \varepsilon \ll \bar{a} \ll \varepsilon_{0} \ll \min (\delta E, 1)$, consider two points $x_{1}^{0}, x_{2}^{0}$ in $\mathbf{T}^{d}$ such that $d\left(x_{1}^{0}, x_{2}^{0}\right) \geq \varepsilon_{0}$ and a velocity $v_{1} \in B_{E}$. Then there exists a subset 
$K\left(x_{1}^{0}-x_{2}^{0}, \varepsilon_{0}, \bar{a}\right)$ of $\mathbf{R}^{d}$ with measure bounded by

$$
\mid\left(K\left(x_{1}^{0}-x_{2}^{0}, \varepsilon_{0}, \bar{a}\right) \mid \leq C E^{d}\left(\left(\frac{\bar{a}}{\varepsilon_{0}}\right)^{d-1}+(E t)^{d} \bar{a}^{d-1}\right)\right.
$$

and a subset $K_{\delta}\left(x_{1}^{0}-x_{2}^{0}, \varepsilon_{0}, \bar{a}\right)$ of $\mathbf{R}^{d}$, the measure of which satisfies

$$
\mid\left(K_{\delta}\left(x_{1}^{0}-x_{2}^{0}, \varepsilon_{0}, \bar{a}\right) \mid \leq C E^{d}\left(\left(\frac{\varepsilon_{0}}{\delta}\right)^{d-1}+(E t)^{d} E^{d-1} \varepsilon_{0}^{d-1}\right)\right.
$$

such that for any $v_{2} \in B_{E}$ and $x_{1}, x_{2}$ such that $\left|x_{1}-x_{1}^{0}\right| \leq \bar{a},\left|x_{2}-x_{2}^{0}\right| \leq \bar{a}$, the following results hold:

- If $v_{1}-v_{2} \notin K\left(x_{1}^{0}-x_{2}^{0}, \varepsilon_{0}, \bar{a}\right)$, then

$$
\forall u \in[0, t], d\left(x_{1}-u v_{1}, x_{2}-u v_{2}\right)>R \varepsilon
$$

and a fortiori, there is no recollision.

- If $v_{1}-v_{2} \notin K_{\delta}\left(x_{1}^{0}-x_{2}^{0}, \varepsilon_{0}, \bar{a}\right)$, then

$$
\forall u \in[\delta, t], d\left(x_{1}-u v_{1}, x_{2}-u v_{2}\right)>\varepsilon_{0},
$$

and so after a time $\delta$, they stay in a good configuration.

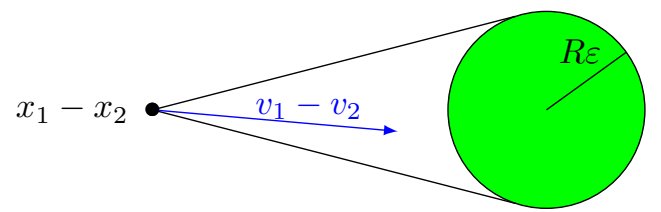

Figure 7. The idea in the proof of Lemma 6.1 is to exclude sets which will lead to pathological situations as illustrated in this picture. Indeed, for relative velocity taken in the cone, the relative positions after transport will belong to the depicted ball of small size, so the particles will be close.

The proof of this lemma in the context of the torus $\mathbf{T}^{d}$ can be found in [7]. Finally, it can be deduced from this result the following property of stability of good configurations by adjunction of an additional particles (see [7]). Let us state it here:

Proposition 6.2. Given $Z_{k}^{0}=\left(X_{k}^{0}, V_{k}^{0}\right) \in \mathcal{G}_{k}\left(\varepsilon_{0}\right)$ and $m_{k} \leq k$, there is a subset $\mathcal{B}_{k}^{m_{k}}\left(Z_{k}^{0}\right)$ of $\mathbf{S}^{d-1} \times B_{E}$ of small measure

$$
\left|\mathcal{B}_{k}^{m_{k}}\left(Z_{k}^{0}\right)\right| \leq C k\left(E^{d}\left(\frac{\bar{a}}{\varepsilon_{0}}\right)^{d-1}+E^{d}(E t)^{d} \varepsilon_{0}^{d-1}+E\left(\frac{\varepsilon_{0}}{\delta}\right)^{d-1}\right)
$$

such that good configurations close to $Z_{k}^{0}$ are stable by adjunction of a collisional particle close to the particle $x_{m_{k}}^{0}$ in the following sense.

Let $Z_{k}=\left(X_{k}, V_{k}\right)$ be a configuration of $k$ particles satisfying

$$
\forall i \leq k\left|x_{i}-x_{i}^{0}\right| \leq \bar{a}, v_{i}=v_{i}^{0} .
$$

Given $\left(\nu_{k+1}, v_{k+1}\right) \in\left(\mathbf{S}^{d-1} \times B_{E}\right) \backslash \mathcal{B}_{k}^{m_{k}}\left(Z_{k}^{0}\right)$, a new particle with velocity $v_{k+1}$ is added at $x_{m_{k}}+R \varepsilon \nu_{k+1}$ to $Z_{k}$ and at $x_{m_{k}}^{0}$ to $Z_{k}^{0}$. 
For a pre-collisional configuration $\nu_{k+1} \cdot\left(v_{k+1}-v_{m_{k}}\right)<0$ then

$$
\forall u \in] 0, t]\left\{\begin{array}{l}
\forall i \neq j \in\{1, \ldots, k\}, d\left(x_{i}-u v_{i}, x_{j}-u v_{j}\right)>R \varepsilon \\
\forall j \in\{1, \ldots, k\}, d\left(x_{m_{k}}+R \varepsilon \nu_{k+1}-u v_{k+1}, x_{j}-u v_{j}\right)>R \varepsilon .
\end{array}\right.
$$

Moreover, after the time $\delta$, the $k+1$ particles are in a good configuration

$$
\forall u \in[\delta, t]\left\{\begin{array}{l}
\left(X_{k}-u V_{k}, V_{k}, x_{m_{k}}+R \varepsilon \nu_{k+1}-u v_{k+1}, v_{k+1}\right) \in \mathcal{G}_{k+1}\left(\varepsilon_{0} / 2\right) \\
\left(X_{k}^{0}-u V_{k}, V_{k}, x_{m_{k}}^{0}+R \varepsilon \nu_{k+1}-u v_{k+1}, v_{k+1}\right) \in \mathcal{G}_{k+1}\left(\varepsilon_{0}\right) .
\end{array}\right.
$$

We will not recall the proof of Proposition 6.2, our concern here will be to rather insist on how we will use it in a slightly different way as usual in the next subsection.

\subsubsection{Inductive construction of the bad sets to remove}

In this previous section, Proposition 6.2 is actually the elementary step for adding a new particle. Let us now iterate it to obtain the construction of the bad sets to remove. The difference in our strategy with what has be done in the previous papers is that since we want to work only with pre-collisional parameters to remove the bad sets, we need to work at each step with updated parameters. In our construction, at stage $k$, we will work with the particles at time $t_{k}-t_{\varepsilon}$, i.e. the $k+1$ particles in their precollisional state. Therefore, when we will truncate on $v_{k+1}\left(t_{k}-t_{\varepsilon}\right)$, it will actually be as if we were truncating in all the previous velocities involved since $v_{k+1}\left(t_{k}-t_{\varepsilon}\right)$ depends on those ones.

We first deal with the initialization of our problem i.e. the construction of geom(2). At this stage, we have one particle, we add a second one and we want to prevent recollisions. We notice here that if the particle lived in $\mathbf{R}^{d}$ we will have nothing to do, but in the case of the torus, there are no longer dispersion properties. So waiting for a sufficiently long time, we expect trajectories to go back $\varepsilon$-close to their initial positions. Then we need to fix an interval of time $[0, t]$ and use Proposition 6.2 to prevent this.

As the construction of the sets geom is interlocked with the iteration strategy, let us recall it here for the first iteration. As explained in Section 3.1, after separating the collisions by a time $\delta$ and getting rid of large velocities, we want to remove collisions in the term

$$
\int_{\mathbf{T}^{d} \times \mathbf{R}^{d}} \varphi(z) \int_{0}^{t-\delta} \mathcal{S}_{1}\left(t-t_{1}\right) \mathcal{C}_{1,2}^{m_{1}} \chi_{H_{2}} \tilde{f}_{N, R}^{(2)}\left(t_{1}, z\right) d t_{1} d z
$$

At this point, we have two possibilities:

- if it is precollisional, we can rewrite the above integral as follows

$$
\begin{array}{r}
\int_{\mathbf{T}^{d} \times \mathbf{R}^{d}} \varphi(z) \int_{0}^{t-\delta} \int_{\mathbf{S}^{d-1} \times \mathbf{R}^{d}}(N-1)(R \varepsilon)^{d-1}\left(\left(v_{2}-v_{m_{1}}\left(t_{1}\right) \cdot \nu_{2}\right)\right)_{-} \\
\tilde{f}_{N, R}^{(1)}\left(t_{2}, \tilde{Z}_{2}\left(t_{1}\right)\right) d \nu_{2} d v_{2} d t_{1} d x_{1} d v_{1} .
\end{array}
$$

Noticing that in the precollisional case $t_{\varepsilon}=0$, we actually have $v_{1}=v_{1}\left(t_{1}-t_{\varepsilon}\right)$ and $v_{2}=$ $v_{2}\left(t_{1}-t_{\varepsilon}\right)$ and idem for $\nu_{2}$ we can rewrite (6.1.8) and obtain

$$
\begin{aligned}
& \int_{\mathbf{T}^{d} \times \mathbf{R}^{d}} \varphi(z) \int_{0}^{t-\delta} \int_{\mathbf{S}^{d-1} \times \mathbf{R}^{d}}(N-1)(R \varepsilon)^{d-1}\left(\left(v_{2}-v_{m_{1}}\left(t_{1}\right) \cdot \nu_{2}\right)\right)_{-} \\
& \tilde{f}_{N, R}^{(1)}\left(t_{2}, \tilde{Z}_{2}\left(t_{1}\right)\right) d \nu_{2}\left(t_{1}-t_{\varepsilon}\right) d v_{2}\left(t_{1}-t_{\varepsilon}\right) d t_{1} d x_{1}\left(t_{1}-t_{\varepsilon}\right) d v_{1}\left(t_{1}-t_{\varepsilon}\right) .
\end{aligned}
$$

Then for $\left(\nu_{2}\left(t_{1}-t_{\varepsilon}\right), v_{2}\left(t_{1}-t_{\varepsilon}\right)\right) \notin B_{1}^{m_{1}}\left(Z_{1}^{0}\right)$, thanks to Proposition 6.2 we know that no recollision occurs. We define geom $(2):=B_{1}^{m_{1}}\left(Z_{1}^{0}\right)$ and we split the above integral into two ones using

$$
1=\chi_{g e o m(2)}\left(\nu_{2}\left(t_{1}-t_{\varepsilon}\right), v_{2}\left(t_{1}-t_{\varepsilon}\right)\right)+\left(1-\chi_{g e o m(2)}\left(\nu_{2}\left(t_{1}-t_{\varepsilon}\right), v_{2}\left(t_{1}-t_{\varepsilon}\right)\right)\right)
$$


where $\chi_{\text {geom(2) }}$ is a regularization of the indicator function of geom(2) defined in (3.1.4), the ensemble geom(2) being regular enough to allow such a regularization (it is an union of cylinders).

Then, noticing that the free transport maps the measure $(R \varepsilon)^{d-1}\left(\left(v_{2}-v_{m_{1}}\left(t_{1}\right) \cdot \nu_{2}\right)\right)_{-} d \nu_{2}\left(t_{1}-\right.$ $\left.t_{\varepsilon}\right) d v_{2}\left(t_{1}-t_{\varepsilon}\right) d t_{1} d x_{1}\left(t_{1}-t_{\varepsilon}\right) d v_{1}\left(t_{1}-t_{\varepsilon}\right)$ on $d x_{2}\left(t_{2}^{+}\right) d v_{2}\left(t_{2}^{+}\right) d x_{1}\left(t_{2}^{+}\right) d v_{1}\left(t_{2}^{+}\right)$, we keep going on the iteration strategy with the latter updated parameters in the part of the integral associated to $1-\chi_{\text {geom(2) }}$.

- If it is post-collisional, we can rewrite (6.1.7) as follows

$$
\begin{gathered}
\int_{\mathbf{T}^{d} \times \mathbf{R}^{d}} \varphi(z) \int_{0}^{t-\delta} \int_{\mathbf{S}^{d-1} \times \mathbf{R}^{d}}(N-1)(R \varepsilon)^{d-1}\left(\left(v_{2}-v_{m_{1}}\left(t_{1}\right) \cdot \nu_{2}\right)\right)_{+} \\
\tilde{f}_{N, R}^{(1)}\left(t_{2}, \tilde{Z}_{2}\left(t_{1}\right)\right) d \nu_{2} d v_{2} d t_{1} d x_{1} d v_{1} .
\end{gathered}
$$

In this case, we actually have $v_{1}=v_{1}\left(t_{1}\right), v_{2}=v_{2}\left(t_{1}\right)$ and idem for $\nu_{2}$. Noticing that the scattering being an involution, it preserves the measure. Thus it maps the measure $d \nu_{2}\left(t_{1}\right) d v_{2}\left(t_{1}\right) d v_{1}\left(t_{1}\right)$ on the measure $d \nu_{2}\left(t_{1}-t_{\varepsilon}\right) d v_{2}\left(t_{1}-t_{\varepsilon}\right) d v_{1}\left(t_{1}-t_{\varepsilon}\right)$. Therefore after doing this change of variables, as previously we can split the integral into two ones using (6.1.10), with $t_{\varepsilon} \neq 0$ this time and we continue as in the pre-collisional case.

We can now deal with the iteration step. Let us assume that we add at time $t_{k}$ the particle $k+1$ knowing that because of the presence of $\left(1-\chi_{\text {geom (2) }}\right)\left(1-\chi_{\text {geom (3) }}\right) \ldots\left(1-\chi_{\text {geom }(k)}\right)$ at this stage, before adding the $(k+1)$-th particle, the $\mathrm{k}$ other particles can not undergo recollisions.

- If it is precollisional, we can split the associated integral directly using

$1=\chi_{g e o m(k+1)}\left(\nu_{k+1}\left(t_{k}-t_{\varepsilon}\right), v_{k+1}\left(t_{k}-t_{\varepsilon}\right)\right)+\left(1-\chi_{\text {geom }(k+1)}\left(\nu_{k+1}\left(t_{k}-t_{\varepsilon}\right), v_{k+1}\left(t_{k}-t_{\varepsilon}\right)\right)\right)$.

with $t_{\varepsilon}=0$. Then, we update our parameters using the fact that the free transport maps the measure $(R \varepsilon)^{d-1}\left(\left(v_{k+1}-v_{m_{k}}\left(t_{k}\right)\right) \cdot \nu_{k+1}\right)_{-} d \nu_{k+1}\left(t_{k}-t_{\varepsilon}\right) d t_{k} d v_{k+1}\left(t_{k}-t_{\varepsilon}\right) d x_{1}\left(t_{k}-t_{\varepsilon}\right) d v_{1}\left(t_{k}-\right.$ $\left.t_{\varepsilon}\right) \ldots d x_{k}\left(t_{k}-t_{\varepsilon}\right) d v_{k}\left(t_{k}-t_{\varepsilon}\right)$ on $d x_{1}\left(t_{k+1}^{+}\right) d v_{1}\left(t_{k+1}^{+}\right) \ldots d x_{k+1}\left(t_{k+1}^{+}\right) d v_{k+1}\left(t_{k+1}^{+}\right)$.

- If it is postcollisional, we use the fact that the scattering preserves the measure. Thus, it maps the measure $d \nu_{k+1}\left(t_{k}\right) d v_{k+1}\left(t_{k}\right) d v_{m_{k}}\left(t_{k}\right)$ on the measure $d \nu_{k+1}\left(t_{k}-t_{\varepsilon}\right) d v_{k+1}\left(t_{k}-t_{\varepsilon}\right) d v_{m_{k}}\left(t_{k}-\right.$ $\left.t_{\varepsilon}\right)$. Then, after doing this change of variables, we conclude as in the precollisional case using (6.1.12) with $t_{\varepsilon} \neq 0$ this time.

\subsubsection{Estimates of the "geometrical" remainders}

We conclude by giving the four last estimates. From now on, we assume that $R, E \geq 1$. The orders of magnitude adopted in Section 7 will satisfy this property.

\subsubsection{Neglecting the pathological trajectories}

As previously, we notice that we can rewrite the term $r_{N}^{R e c o l l, K}(t)$ as follows

$$
r_{N}^{\text {Recoll }, K}(t)=\sum_{k=1}^{K} \sum_{j_{1}=0}^{n_{1}-1} \cdots \sum_{j_{k}=0}^{n_{k}-1} F^{\text {Recoll }}\left(J^{(k)}, z\right)
$$

where for $J^{(k)}=\left(1, j_{1}, \ldots, j_{k}\right)$ fixed, we denote

$$
\begin{aligned}
F^{\operatorname{Recoll}}\left(J^{(k)}, z\right) & :=Q_{1, J_{1}}(\tau) \ldots Q_{J_{k-2}, J_{k-1}}(\tau) \\
& \int_{0}^{\tau-j_{k} \delta} Q_{J_{k-1}, J_{k}}\left(\tau-t_{J_{k}}\right) \mathcal{C}_{J_{k}, J_{k}+1} \chi_{H_{J_{k}+1}} \chi_{g e o m\left(J_{k}+1\right)} \tilde{f}_{N, R}^{\left(J_{k}+1\right)}\left(t_{J_{k}}, z\right) d t_{J_{k}} .
\end{aligned}
$$

Then we get the following bound 
Proposition 6.3. Let $k$ be an integer, $1 \leq k \leq K$. There exists a constant $C$ such that for any $\tau>0, J=\left(j_{1}, \ldots, j_{k-1}\right)$ with $j_{i} \in\left\{0, \ldots, n_{i}-1\right\}$ for all $i$,

$$
\begin{aligned}
\left|\int_{\mathbf{R}^{d} \times \mathbf{T}^{d}} \varphi(z) F^{\text {Recoll }}\left(J^{(k)}, z\right) d z\right| \leq C 2^{K+1}\left(\tilde{C} R^{d-1} t\right)^{2^{K+1}} & \\
& \left(E^{d}\left(\frac{\bar{a}}{\varepsilon_{0}}\right)^{d-1}+E^{d}(E t)^{d} \varepsilon_{0}^{d-1}+E\left(\frac{\varepsilon_{0}}{\delta}\right)^{d-1}\right) .
\end{aligned}
$$

Proof. The proof is quite similar to the ones of the remainders in Section 5 and is based on using continuity estimates. Noticing the three following points leads to the conclusion:

- As previously, the exact distribution of collisions in the last $k$ intervals is not needed.

- Concerning the last collision operator involved, $\mathcal{C}_{J_{k}, J_{k}+1}$, similarly as in the proof of the usual continuity estimates in Appendix C, the integration with respect to velocity brings a factor $(2 \pi / \beta)^{d / 2}$ while the presence of $\chi_{\text {geom }\left(J_{k}+1\right)}$ gives a factor

$$
C J_{k}\left(E^{d}\left(\frac{\bar{a}}{\varepsilon_{0}}\right)^{d-1}+E^{d}(E t)^{d} \varepsilon_{0}^{d-1}+E\left(\frac{\varepsilon_{0}}{\delta}\right)^{d-1}\right)
$$

according to Propositon 6.2.

- Finally, $J_{k}=1+j_{1}+\cdots+j_{k} \leq 1+n_{1}+\cdots+n_{k}=\frac{2^{k+1}-1}{2-1} \leq 2^{k+1} \leq 2^{K+1}$.

Then we have

$$
\begin{aligned}
& \left|\int_{\mathbf{R}^{d} \times \mathbf{T}^{d}} \varphi(z) F^{\text {Recoll }}\left(J^{(k)}, z\right) d z\right| \\
& \leq C\|\varphi\|_{\infty}\left(\frac{R^{d-1} C_{d} k \tau}{\beta^{(d+1) / 2}}\right)^{J_{k}} 2^{K+1}\left(E^{d}\left(\frac{\bar{a}}{\varepsilon_{0}}\right)^{d-1}+E^{d}(E t)^{d} \varepsilon_{0}^{d-1}+E\left(\frac{\varepsilon_{0}}{\delta}\right)^{d-1}\right) \\
& \quad \sup _{t \geq 0}\left\|\tilde{f}_{N, R}^{\left(J_{k}+1\right)}(t)\right\|_{\varepsilon, J_{k}, \beta} \\
& \leq C 2^{K+1}\left(\tilde{C} R^{d-1} t\right)^{2^{K+1}}\left(E^{d}\left(\frac{\bar{a}}{\varepsilon_{0}}\right)^{d-1}+E^{d}(E t)^{d} \varepsilon_{0}^{d-1}+E\left(\frac{\varepsilon_{0}}{\delta}\right)^{d-1}\right)
\end{aligned}
$$

which concludes the proof.

\subsection{Time separation}

We can rewrite the term $r_{N}^{\text {Tim,K }}(t)$ as follows

$$
r_{N}^{T i m, K}(t)=\sum_{k=1}^{K} \sum_{j_{1}=0}^{n_{1}-1} \cdots \sum_{j_{k}=0}^{n_{k}-1} F^{T i m}\left(J^{(k)}, z\right)
$$

where for $J^{(k)}=\left(1, j_{1}, \ldots, j_{k}\right)$ fixed, we denote

$$
F^{T i m}\left(J^{(k)}, z\right):=Q_{1, J_{1}}(\tau) \ldots Q_{J_{k-2}, J_{k-1}}(\tau) \int_{0}^{\tau-j_{k} \delta} Q_{J_{k-1}, J_{k}}^{\delta}\left(\tau-t_{J_{k}}\right) \mathcal{C}_{J_{k}, J_{k}+1} \tilde{f}_{N, R}^{\left(J_{k}+1\right)}\left(t_{J_{k}}, z\right) d t_{J_{k}}
$$

Proposition 6.4. Let $k$ be an integer, $1 \leq k \leq K$. There exists a constant $C$ such that for any $\tau>0, J=\left(j_{1}, \ldots, j_{k-1}\right)$ with $j_{i} \in\left\{0, \ldots, n_{i}-\overline{1}\right\}$ for all $i$,

$$
\left|\int_{\mathbf{R}^{d} \times \mathbf{T}^{d}} \varphi(z) F^{T i m}\left(J^{(k)}, z\right) d z\right| \leq C\left(C R^{d-1} t\right)^{2^{K+1}} 2^{K+1} \frac{\delta}{t} .
$$


Proof. The reasoning is exactly the same as the one in the proof of the continuity estimates except that it involves the integration over two consecutive times such that $\left|t_{J_{k}-1}-t_{J_{k}}\right| \leq \delta$. Consequently, the integration with respect to time provides a factor $\frac{\delta t^{J_{k}-1}}{\left(J_{k}-1\right) !}$ instead of $\frac{t^{J_{k}}}{\left(J_{k}\right) !}$ in the usual proof.

Using the Stirling formula as in the proof of the classical continuity estimates together with the a priori estimates on the truncated marginals and the fact that $J_{k}+1 \leq 2^{k+1} \leq 2^{K+1}$ leads to the conclusion.

\subsection{Energy truncation}

We can rewrite the term $r_{N}^{\text {Ener, } K}(t)$ as follows

$$
r_{N}^{E n e r, K}(t)=\sum_{k=1}^{K} \sum_{j_{1}=0}^{n_{1}-1} \cdots \sum_{j_{k}=0}^{n_{k}-1} F^{E n e r}\left(J^{(k)}, z\right)
$$

where for $J^{(k)}=\left(1, j_{1}, \ldots, j_{k}\right)$ fixed, we denote

$$
\begin{aligned}
F^{\text {Ener }}\left(J^{(k)}, z\right):= & Q_{1, J_{1}}(\tau) \ldots Q_{J_{k-2}, J_{k-1}}(\tau) \\
& \int_{0}^{\tau-j_{k} \delta} Q_{J_{k-1}, J_{k}}\left(\tau-t_{J_{k}}\right) \mathcal{C}_{J_{k}, J_{k}+1}\left(1-\chi_{H_{J_{k}+1}}\right) \tilde{f}_{N, R}^{\left(J_{k}+1\right)}\left(t_{J_{k}}, z\right) d t_{J_{k}} .
\end{aligned}
$$

Proposition 6.5. Let $k$ be an integer, $1 \leq k \leq K$. There exists a constant $C$ such that for any $\tau>0, J=\left(j_{1}, \ldots, j_{k-1}\right)$ with $j_{i} \in\left\{0, \ldots, n_{i}-1\right\}$ for all $i$,

$$
\left|\int_{\mathbf{R}^{d} \times \mathbf{T}^{d}} \varphi(z) F^{E n e r}\left(J^{(k)}, z\right) d z\right| \leq C e^{-\frac{\beta}{4} E^{2}}\left(C R^{d-1} t\right)^{2^{K+1}} .
$$

Proof. Using the obvious following inequality

$$
\sup _{t \geq 0}\left\|\left(1-\chi_{\left\{H_{J_{k}+1}\left(Z_{J_{k}+1}\right) \leq E^{2}\right\}}\right) \tilde{f}_{N, R}^{\left(J_{k}+1\right)}(t)\right\|_{\varepsilon, J_{k}+1, \beta} \leq e^{-\frac{\beta}{4} E^{2}}\left\|\tilde{f}_{N, R}^{\left(J_{k}+1\right)}(t)\right\|_{\varepsilon, J_{k}+1, \beta}
$$

and the same arguments as previously leads to the result.

\subsection{Small relative velocities}

We can rewrite the term $r_{N}^{\text {Relat.vel,K}}(t)$ as follows

$$
r_{N}^{\text {Relat.vel }, K}(t)=\sum_{k=1}^{K} \sum_{j_{1}=0}^{n_{1}-1} \cdots \sum_{j_{k}=0}^{n_{k}-1} F^{\text {Relat.vel }}\left(J^{(k)}, z\right)
$$

where for $J^{(k)}=\left(1, j_{1}, \ldots, j_{k}\right)$ fixed, we denote

$$
\begin{aligned}
& F^{\text {Relat.vel }}\left(J^{(k)}, z\right):=Q_{1, J_{1}}(\tau) \ldots Q_{J_{k-2}, J_{k-1}}(\tau) \\
& \quad \int_{0}^{\tau-j_{k} \delta} Q_{J_{k-1}, J_{k}}\left(\tau-t_{J_{k}}\right) \mathcal{C}_{J_{k}, J_{k}+1} \chi_{H_{J_{k}+1}}\left(1-\chi_{g e o m\left(J_{k}+1\right)}\right)\left(1-\chi_{\eta_{J_{k}+1}}\right) \tilde{f}_{N, R}^{\left(J_{k}+1\right)}\left(t_{J_{k}}, z\right) d t_{J_{k}} .
\end{aligned}
$$

Proposition 6.6. Let $k$ be an integer, $1 \leq k \leq K$. There exists a constant $C$ such that for any $\tau>0, J=\left(j_{1}, \ldots, j_{k-1}\right)$ with $j_{i} \in\left\{0, \ldots, n_{i}-1\right\}$ for all $i$,

$$
\left|\int_{\mathbf{R}^{d} \times \mathbf{T}^{d}} \varphi(z) F^{\text {Relat.vel }}\left(J^{(k)}, z\right) d z\right| \leq C\left(C R^{d-1} t\right)^{2^{K+1}} 2^{2(K+1)} \eta^{d} .
$$


Proof. First of all, let us notice that

$$
\prod_{i=1}^{J_{k}} \mathbf{1}_{\left|v_{i}-v_{J_{k}+1}\right| \geq \eta} \leq \chi_{\eta_{J_{k}+1}}
$$

and so we have the following inequality

$$
1-\chi_{\eta_{J_{k}+1}} \leq \mathbf{1}-\prod_{i=1}^{J_{k}} \mathbf{1}_{\left|v_{i}-v_{J_{k}+1}\right| \geq \eta} \leq \sum_{i=1}^{J_{k}} \mathbf{1}_{\left|v_{i}-v_{J_{k}+1}\right| \leq \eta} .
$$

The reasoning is then quite similar to the previous ones except that because of the presence of $\sum_{i=1}^{J_{k}} \mathbf{1}_{\left|v_{i}-v_{J_{k}+1}\right| \leq \eta}$, the last collision operator will be bounded using the following inequality: for all $i \in\left\{1, \ldots, J_{k}\right\}$,

$$
\begin{array}{r}
\int_{\mathbf{R}^{d}}\left(\left|v_{J_{k}+1}\right|+\left|v_{i}\right|\right) \exp \left(-\frac{\beta}{2} \sum_{l=1}^{J_{k}+1}\left|v_{l}\right|^{2}\right) \sum_{j=1}^{J_{k}} \mathbf{1}_{\left|v_{j}-v_{J_{k}+1}\right| \leq \eta} d v_{J_{k}+1} \\
\leq C J_{k} \eta^{d} \exp \left(-\frac{\beta}{2} \sum_{l=1}^{J_{k}+1}\left|v_{l}\right|^{2}\right) .
\end{array}
$$

Then the same arguments as in the proof of the continuity estimates together with the a priori estimates on $\tilde{f}_{N, R}$ and the fact that $J_{k} \leq 2^{K+1}$ leads to the conclusion.

\section{$7 \quad$ Asymptotic vanishing of the remainders}

It is now time to prove in this section that the remainders will vanish asymptotically provided that the parameters are correctly chosen.

Proposition 7.1. For $\varphi$ a test function, for $\delta, \varepsilon_{0}, E, \bar{a}, \eta, K$ and $R$ satisfying the following orders of magnitude:

$$
\begin{gathered}
\delta=\varepsilon^{\frac{d-1}{d+1}}, \varepsilon_{0}=\varepsilon^{d /(d+1)}, E=C \sqrt{|\log \varepsilon|}, \bar{a}=2^{K+1} \varepsilon, \eta=\frac{1}{|\log \varepsilon|}, \\
\frac{\log \left(\log |\log \varepsilon|^{1 / 4}\right)}{\log 2} \leq \tilde{C}^{2} R^{2(d-1)}
\end{gathered}
$$

and

$$
2(K+1) \leq \frac{\log \left(\log |\log \varepsilon|^{1 / 2}\right)}{\log 2},
$$

then

$$
\left|\int_{\mathbf{R}^{d} \times \mathbf{T}^{d}} \varphi(z) r_{N}^{K}(t, z) d z\right| \underset{N \rightarrow \infty}{\longrightarrow} 0 .
$$

Proof. Let us prove that they vanish in the right order. We start with $r^{\text {Supexp }}$. We choose $\tau \leq \frac{\gamma}{\left(\tilde{C} R^{d-1}\right)^{2} t}$ with $\left.\gamma \in\right] 0,1\left[\right.$. Thanks to Proposition 5.1, after summing on $k$ and $j_{1}, \ldots, j_{k}$, we get the following bound

$$
\begin{aligned}
\left|\int_{\mathbf{R}^{d} \times \mathbf{T}^{d}} \varphi(z) r_{N}^{\text {Supexp }, K}(t, z) d z\right| & \leq C \sum_{k=1}^{K} 2^{k(k+1)}\left[\left(\tilde{C} R^{d-1}\right)^{2} t \tau\right]^{2^{k}} \\
& \leq C \sum_{k=1}^{K} 2^{k(k+1)} \gamma^{2^{k}} \\
& =C \sum_{k=1}^{K} \exp \left(k(k+1) \log 2+2^{k} \log \gamma\right) \\
& \leq C \sum_{k=1}^{K} \exp (2 k \log \gamma) \\
& =C \sum_{k=1}^{K}\left(\gamma^{2}\right)^{k} \\
& \leq c \gamma^{2} .
\end{aligned}
$$


This relation being true for any $\gamma \in] 0,1[$ proves that this term vanish asymptotically. Moreover, by summing on $k$ and $j_{1}, \ldots, j_{k}$ with the orders of magnitude chosen for the parameters, we get the following bound for $\alpha>0$ small enough,

$$
\begin{array}{r}
\left|\int_{\mathbf{R}^{d} \times \mathbf{T}^{d}} \varphi(z)\left(r_{N}^{\text {Recoll }, K}(t, z)+r_{N}^{\text {Tim }, K}(t, z)+r_{N}^{\text {Ener }, K}(t, z)+r_{N}^{C l u, K}(t, z)\right) d z\right| \\
\leq C K 2^{(K+2)(K+1)}\left(\left(C R^{d-1} t\right)^{2}\right)^{2^{K}} \varepsilon^{\alpha}
\end{array}
$$

as a direct consequence of Propositions 5.3, 6.3, 6.4, 6.5.

Let us take twice the logarithm on the right-hand side of (7.0.12). Then, the higher order term in $K$ is $K \log 2$. Using the idea that if $A(a) \underset{a \rightarrow 0}{\sim} B(a)$ then $A(a) \leq 2 B(a)$ for a small enough, then for $\varepsilon$ small enough, we get the following inequality

$$
\left|\int_{\mathbf{R}^{d} \times \mathbf{T}^{d}} \varphi(z)\left(r_{N}^{\text {Recoll }, K}(t, z)+r_{N}^{T i m, K}(t, z)+r_{N}^{\text {Ener }, K}(t, z)+r_{N}^{C l u, K}(t, z)\right) d z\right| \leq C \exp \left(2^{2 K}\right) \varepsilon^{\alpha} .
$$

Consequently, by assumption (7.0.9) we can deduce that

$$
2 K \leq \frac{\log |\log \varepsilon|^{1 / 2}}{\log 2}
$$

and we get

$$
2^{2 K} \leq|\log \varepsilon|^{1 / 2}
$$

Thus finally,

$$
\begin{gathered}
\left|\int_{\mathbf{R}^{d} \times \mathbf{T}^{d}} \varphi(z)\left(r_{N}^{\text {Recoll }, K}(t, z)+r_{N}^{\text {Tim, }, K}(t, z)+r_{N}^{\text {Ener }, K}(t, z)+r_{N}^{C l u, K}(t, z)\right) d z\right| \\
\leq C e^{|\log \varepsilon|^{1 / 2}} \varepsilon^{\alpha}=C e^{|\log \varepsilon|^{1 / 2}+\alpha \log \varepsilon} \underset{\varepsilon \rightarrow 0}{\longrightarrow} 0 .
\end{gathered}
$$

Moreover a similar argument shows that, for $\varepsilon$ small enough,

$$
\left|\int_{\mathbf{R}^{d} \times \mathbf{T}^{d}} \varphi(z) r_{N}^{\text {Relat.vel, } K}(t, z) d z\right| \leq C \exp \left(2^{2^{K}}\right) \eta^{d}
$$

as a consequence of Proposition 6.6. Then by assumption, we get

$$
2 K \leq \frac{\log \left(\log |\log \varepsilon|^{1 / 2}\right)}{\log 2}
$$

and so

$$
2^{2 K} \leq \log |\log \varepsilon|^{1 / 2}
$$

Therefore finally,

$$
\begin{aligned}
&\left|\int_{\mathbf{R}^{d} \times \mathbf{T}^{d}} \varphi(z) r_{N}^{\text {Relat.vel,K}}(t, z) d z\right| \\
& \leq C e^{\log |\log \varepsilon|^{1 / 2}}\left(\frac{1}{|\log \varepsilon|}\right)^{d}=C \frac{|\log \varepsilon|^{1 / 2}}{|\log \varepsilon|^{d}} \underset{\varepsilon \rightarrow 0}{\longrightarrow} 0 .
\end{aligned}
$$

Let us observe the two last terms. As a consequence of Propositions 4.4 and 4.5, after summing 
on $k$ and $j_{1}, \ldots, j_{k}$, we have the following bound:

$$
\begin{aligned}
\mid \int_{\mathbf{R}^{d} \times \mathbf{T}^{d}} \varphi(z) & \left(r_{N}^{P o t, a, K}(t, z)+r_{N}^{P o t, b, K}(t, z)\right) d z \mid \\
\leq & C K 2^{(K+1)^{2}}\left(R^{d-1} E^{d+1} t\right)^{2^{K+1}} \frac{1}{\varepsilon^{d-1}}\left[\left(\frac{C R e^{C \frac{R^{2}}{\eta}}}{\eta\left|\cos \left(\frac{\pi}{2}-\varepsilon\right)\right| \varepsilon}\right)^{2^{K+1}}+\frac{2^{K+1}}{\varepsilon}\right]\left\|\nabla \Phi^{>}\right\|_{\infty} \\
\leq & C K 2^{(K+1)^{2}}\left(R^{d-1} E^{d+1} t\right)^{2^{K+1}} \frac{1}{\varepsilon^{d-1}}\left(\frac{C R e^{C \frac{R^{2}}{\eta}}}{\eta\left|\cos \left(\frac{\pi}{2}-\varepsilon\right)\right| \varepsilon}\right)^{2^{K+1}}\left\|\nabla \Phi^{>}\right\|_{\infty} .
\end{aligned}
$$

with the order of magnitude (7.0.7), (7.0.8) and (7.0.9) for $\varepsilon$ small enough. By a reasoning quite similar to the previous one, we know that it is enough to conclude to prove the vanishing of the higher order term $\left(e^{C \frac{R^{2}}{\eta}}\right)^{2^{K+1}}$ against $\|\nabla \Phi\|_{\infty}$ as $\varepsilon$ goes to 0 , the order of the other terms not being large enough to change this convergence.

We denote $\Gamma(x):=\exp \left(-\exp \left(12 \exp \left(\tilde{C}^{2}|x|^{2(d-1)} \log 2\right)\right)\right)$. By Assumption 4.1, $\nabla \Phi$ decreases at least like $\Gamma$ for the appropriate choice of $\lambda$ in (4.0.7). Then,

$$
\begin{aligned}
\left(e^{C \frac{R^{2}}{\eta}}\right)^{2^{K+1}} \| & \nabla \Phi \|_{\infty} \\
& \leq e^{C 2^{K+1}\left[\left(\log \left(\log |\log \varepsilon|^{1 / 2}\right)\right)^{2}|\log \varepsilon|\right]} \exp \left(-\exp \left(12 \exp \left(\tilde{C}^{2} R^{2(d-1)} \log 2\right)\right)\right) \\
& \leq e^{C \log |\log \varepsilon|^{1 / 2}\left(\log \left(\log |\log \varepsilon|^{1 / 2}\right)\right)^{2}|\log \varepsilon|} \exp \left(-\exp \left(12 \exp \left(\log \left(\log |\log \varepsilon|^{1 / 4}\right)\right)\right)\right) \\
& \leq e^{C\left(\log |\log \varepsilon|^{1 / 2}\right)^{3}|\log \varepsilon|} \exp \left(-|\log \varepsilon|^{3}\right) \\
& \leq e^{C|\log \varepsilon|^{5 / 2}-|\log \varepsilon|^{3}} \underset{\varepsilon \rightarrow 0}{\longrightarrow} 0
\end{aligned}
$$

which concludes the proof.

\section{Convergence to the Boltzmann with cut-off equation}

\subsection{The Boltzmann with cut-off series}

The dynamics associated to the Boltzmann with cut-off hierarchy are governed by the following integral equations

$$
g_{R}^{(s)}(t)=\mathcal{S}_{s}^{0}(t) g_{R}^{(s)}(0)+\int_{0}^{t} \mathcal{S}_{s}^{0}(t-\tau) \mathcal{C}_{s, s+1}^{0, R} g_{R}^{(s+1)}(\tau) d \tau
$$

where $\mathcal{S}_{s}^{0}$ denotes the free flow of $s$ particles on $\mathbf{T}^{d s} \times \mathbf{R}^{d s}$ and $\mathcal{C}_{s, s+1}^{0, R}$ represents the collision operator defined as follows:

$$
\begin{aligned}
&\left(\mathcal{C}_{s, s+1}^{0, R} f^{(s+1)}\right)\left(Z_{s}\right):=R \sum_{i=1}^{s} \int_{\mathbf{S}^{d-1} \times \mathbf{R}^{d}} \mathbf{1}_{\nu \cdot\left(v_{s+1}-v_{i}\right)>0}\left(\nu \cdot\left(v_{s+1}-v_{i}\right)\right) \\
& {\left[f^{(s+1)}\left(\ldots, x_{i}, v_{i}^{* R}, \ldots, x_{i}, v_{s+1}^{* R}\right)\left(\nu \cdot\left(v_{s+1}-v_{i}\right)\right) d \nu d v_{s+1}\right.} \\
&\left.-f^{(s+1)}\left(\ldots, x_{i}, v_{i}, \ldots, x_{i}, v_{s+1}\right)\right] d \nu d v_{s+1}
\end{aligned}
$$

where $\left(v_{i}^{* R}, v_{s+1}^{* R}\right)$ are obtained from $\left(v_{i}, v_{s+1}\right)$ applying the inverse scattering operator corresponding to the Boltzmann with cut-off case. We will denote this scattering operator by $\sigma_{R}$.

We put

$$
g^{0(s)}\left(Z_{s}\right):=\rho^{0}\left(x_{1}\right) M_{\beta}^{\otimes s}\left(V_{s}\right)
$$


where we recall $M_{\beta}(v)=\left(\frac{\beta}{2 \pi}\right)^{d / 2} \exp \left(-\frac{\beta}{2}|v|^{2}\right)$.

The iteration strategy adopted previously can also be applied here. From now on, we will denote $g_{R}^{(1)}$ by $g_{R}$. We obtained then the following expansion for $g_{R}$ :

$$
\int_{\mathbf{T}^{d} \times \mathbf{R}^{d}} \varphi(z) g_{R}(t, z) d z=\int_{\mathbf{T}^{d} \times \mathbf{R}^{d}} \varphi(z) g_{R}^{(1, K)}(t, z) d z+\int_{\mathbf{T}^{d} \times \mathbf{R}^{d}} \varphi(z) r_{R}^{0, K}(t, z) d z
$$

where

$$
\begin{gathered}
g_{R}^{(1, K)}(t):=\sum_{j_{1}=0}^{n_{1}-1} \cdots \sum_{j_{K}=0}^{n_{K}-1} Q_{1, J_{1}}^{0, R}(\tau) \ldots Q_{J_{K-1}, J_{K}}^{0, R}(\tau) g_{R}^{0\left(J_{K}\right)}, \\
r_{R}^{0, K}(t):=\sum_{k=1}^{K} \sum_{j_{1}=0}^{n_{1}-1} \cdots \sum_{j_{k-1}=0}^{n_{k-1}-1} Q_{1, J_{1}}^{0, R}(\tau) \ldots Q_{J_{k-2}, J_{k-1}}^{0, R}(\tau) r_{J_{k-1}, n_{k}}^{0, R}(t-k \tau, t-(k-1) \tau)
\end{gathered}
$$

with $Q_{s, s+n}^{0, R}(t)$ and

$$
r_{J_{k-1}, n_{k}}^{0, R}:=r_{J_{k-1}, n_{k}}^{0, R, \text { Tim }}+r_{J_{k-1}, n_{k}}^{0, R, \text { Ener }}+r_{J_{k-1}, n_{k}}^{0, R, \text { Recoll }}+r_{J_{k-1}, n_{k}}^{0, R, \text { Relat.vel }}+r_{J_{k-1}, n_{k}}^{0, R, \text { Supexp }}
$$

defined similarly as in the case of the BBGKY hierarchy.

Remark 8.1. The notation $r_{J_{k-1}, n_{k}}^{0, R, \text { Reoll }}$ is not actually appropriate since there is no recollision in the Boltzmann case. Yet, by this we only mean that we do the truncation $\chi_{\text {geom }}$ associated to situations where there may be recollisions in the BBGKY case.

In the following, we will state the results associated to this series without giving the proof, the proof being essentially the same ones as in the case of the BBGKY series. Let us first start with the continuity estimates.

Lemma 8.1. There is a constant $C_{d}$ depending only on $d$ such that for all $s, n \in \mathbf{N}, t \geq 0$, the operators $|Q|_{s, s+n}^{0, R}(t)$ (defined similarly as $|Q|_{s, s+n}(t)$ ) satisfy the following continuity estimates: for all $f_{s+n} \in X_{\varepsilon, s+n, \alpha},|Q|_{s, s+n}^{0, R}(t) f_{s+n}$ belongs to $X_{\varepsilon, s, \frac{\alpha}{2}}$ and

$$
\left\||Q|_{s, s+n}^{0, R}(t) f_{s+n}\right\|_{\varepsilon, s, \frac{\alpha}{2}} \leq e^{s-1}\left(\frac{C_{d} R^{d-1} t}{\alpha^{\frac{d+1}{2}}}\right)^{n}\left\|f_{s+n}\right\|_{\varepsilon, s+n, \alpha} .
$$

Moreover, applying the maximum principle for the linear Boltzmann equation with cut-off, we obtain the following result:

Proposition 8.1. For any $s \geq 1$, we have the following uniform bound (with respect to time)

$$
\sup _{t \geq 0} g_{R}^{(s)}\left(t, Z_{s}\right) \leq\left\|\rho^{0}\right\|_{\infty} M_{\beta}^{\otimes s}\left(V_{s}\right) .
$$

Then, following the same argument as in the previous sections, we can establish the below result:

Proposition 8.2. For $\varphi$ a test function, for $\delta, \varepsilon_{0}, E, \bar{a}, \eta, K$ and $R$ satisfying the orders of magnitude stated in (7.0.7), (7.0.8) and (7.0.9), then

$$
\left|\int_{\mathbf{R}^{d} \times \mathbf{T}^{d}} \varphi(z) r_{R}^{0, K}(t, z) d z\right| \underset{N \rightarrow \infty}{\longrightarrow} 0 .
$$




\subsection{Convergence}

As mentioned previously, one of our main aim in order to get the convergence is to couple the pseudo-trajectories. Actually, a direct corollary of Proposition 6.2 allows us to state the following result:

Proposition 8.3. Fix $J:=\left(j_{1}, \ldots, j_{K}\right), m=\left(m_{1}, \ldots, m_{J_{K}-1}\right)$ with $m_{i} \leq i$. We consider the pseudo-trajectories $\tilde{Z}_{i}, \tilde{Z}_{i}^{R, 0}$ defined inductively by choosing at each collision time $t_{i}$ a deflection angle $\nu_{i+1}$ and a velocity $v_{i+1}$ such that $\left(\nu_{i+1}, v_{i+1}\right) \in \mathbf{S}^{d-1} \times B_{E} \backslash \mathcal{B}_{i}^{m_{i}}\left(Z_{i}^{R, 0}\left(t_{i}\right)\right)$.

Then, in our context (low energy, collisions being separated by at least $\delta$ and a low bound on the relative velocities), the velocities of both pseudo-trajectories coincides as well as the positions $x_{1}(u)=x_{1}^{0, R}(u)$ for $u \in[0, t]$. Furthermore, for $\varepsilon$ sufficiently small $\forall i \leq J_{K}-1, \forall l \leq i+1$,

$$
\left|x_{l}\left(t_{i+1}\right)-x_{l}^{0, R}\left(t_{i+1}\right)\right| \leq \varepsilon i .
$$

The proof is quite straightforward reasoning by induction on $i$ and can be found in [7].

Let us now bring the attention on the fact that, as it can be seen in the expression of the main term, there is a special role played by initial data. Actually, they do not only present the advantage to provide some a priori estimates via the maximum principle as stated in Proposition 4.1 but they also lead to an asymptotical factorisation (see Appendix B for the proof).

Proposition 8.4. For the initial data $f_{N}$ given in (2.1.1), the truncated marginal of order $s$

$$
\tilde{f}_{N}^{(s)}:=\int f_{N}^{0}\left(Z_{N}\right) \prod_{\substack{1 \leq i \leq s \\ s+1 \leq j \leq N}} \mathbf{1}_{\left\{\left|x_{i}-x_{j}\right|>R \varepsilon\right\}} d Z_{(s+1, N)}=\rho^{0}\left(x_{1}\right) \tilde{M}_{N, \beta}^{(s)}\left(Z_{s}\right)
$$

converges as $N$ goes to $\infty$, under the Boltzmann-Grad scaling $N \varepsilon^{d-1}=1$, to the function $g^{0(s)}$ defined in (8.1.3).

We finally have all the tools to state the following result:

Proposition 8.5. For $\varphi$ a test function, for $\delta, \varepsilon_{0}, E, \bar{a}, \eta, K$ and $R$ satisfying the orders of magnitude stated in (7.0.7), (7.0.8) and (7.0.9), we have

$$
\sup _{t \geq 0}\left\|\tilde{f}_{N, R}^{(1, K)}(t)-g_{R}^{(1, K)}(t)\right\|_{L^{\infty}\left(\mathbf{T}^{d} \times \mathbf{R}^{d}\right)}^{\longrightarrow} \underset{R \rightarrow \infty}{\longrightarrow} 0 .
$$

Proof. We denote $\mathcal{T}_{J, \delta}(\tau):=\left\{T=\left(t_{1}, \ldots, t_{J_{K}-1}\right) \mid t_{i}<t_{i-1}-\delta,\left(t_{J_{k}}, \ldots, t_{J_{k-1}+1}\right) \in[t-k \tau, t-(k-\right.$ 1) $\tau]\}, \mathcal{M}_{J}:=\left\{m=\left(m_{1}, \ldots, m_{J_{K}-1}\right), 1 \leq m_{i} \leq i\right\}, J:=\left(j_{1}, \ldots, j_{K}\right)$.

Then, we can rewrite $\tilde{f}_{N, R}^{(1, K)}$ and $g_{R}^{(1, K)}$ as follows

$$
\tilde{f}_{N, R}^{(1, K)}(t, z)=\sum_{J}(R \varepsilon)^{(d-1)\left(J_{K}-1\right)} \frac{(N-1) !}{\left(N-J_{K}\right) !} \sum_{m \in \mathcal{M}_{J}} \Xi_{N, R}^{(1, K)}(J, m)
$$

and

$$
g_{R}^{(1, K)}(t, z)=\sum_{J} R^{(d-1)\left(J_{K}-1\right)} \sum_{m \in \mathcal{M}_{J}} \Upsilon_{N, R}^{(1, K)}(J, m)
$$

where denoting by $\chi$ the product of all the smooth indicator functions which are involved,

$$
\begin{aligned}
\Xi_{N, R}^{(1, K)}(J, m):=\int_{\mathcal{T}_{J, \delta(\tau)}} d T \int_{\left(\mathbf{S}^{d-1} \times B_{E}\right)^{J_{K}-1}} d \nu_{1} \ldots d \nu_{J_{K}} d v_{2} \ldots d v_{J_{K}} \chi \\
\\
\prod_{i=1}^{J_{K}-1}\left(\nu_{i+1} \cdot\left(v_{i+1}-v_{m_{i}}\left(t_{i}\right)\right)\right) \tilde{f}_{N, R}^{0\left(J_{K}\right)}\left(Z_{J_{K}}(0)\right)
\end{aligned}
$$


and

$$
\begin{array}{rl}
\Upsilon_{R}^{(1, K)}(J, m):=\int_{\mathcal{T}_{J, \delta(\tau)}} d T \int_{\left(\mathbf{S}^{d-1} \times B_{E}\right)^{J_{K}-1}} & d \nu_{1} \ldots d \nu_{J_{K}} d v_{2} \ldots d v_{J_{K}} \chi \\
& \prod_{i=1}^{J_{K}-1}\left(\nu_{i+1} \cdot\left(v_{i+1}-v_{m_{i}}\left(t_{i}\right)\right)\right) g_{R}^{0\left(J_{K}\right)}\left(Z_{J_{K}}^{0, R}(0)\right) .
\end{array}
$$

Then the only differences between those two series are the prefactors $\varepsilon^{(d-1)\left(J_{K}-1\right)} \frac{(N-1) !}{\left(N-J_{K}\right) !}$, the small error on the positions $Z_{J_{K}}(0)$ and $Z_{J_{K}}^{0, R}(0)$ and the initial data $\tilde{f}_{N, R}^{0\left(J_{K}\right)}$ and $g_{R}^{0\left(J_{K}\right)}$. Using the identity $N \varepsilon^{d-1}=1$, we can prove that the prefactors converges to 1 at infinity. Moreover, we have the following identity

$$
\begin{aligned}
& \left|\tilde{f}_{N, R}^{0\left(J_{K}\right)}\left(Z_{J_{K}}(0)\right)-g_{R}^{0\left(J_{K}\right)}\left(Z_{J_{K}}^{0, R}(0)\right)\right| \\
& \quad \leq\left|\tilde{f}_{N, R}^{0\left(J_{K}\right)}\left(Z_{J_{K}}(0)\right)-g_{R}^{0\left(J_{K}\right)}\left(Z_{J_{K}}(0)\right)\right|+\left|g_{R}^{0\left(J_{K}\right)}\left(Z_{J_{K}}(0)\right)-g_{R}^{0\left(J_{K}\right)}\left(Z_{J_{K}}^{0, R}(0)\right)\right|
\end{aligned}
$$

Then using Proposition 8.3 and Proposition 8.4 together with the continuity of $g_{R}^{0}$ leads to the conclusion.

Finally, the convergence to 0 of the difference between the truncated marginals and the marginals can be obtained as stated in the following proposition that is proven in Appendix B.

Proposition 8.6. For the initial data $f_{N}^{0}$ given in (2.1.1), for all $t \geq 0$, the difference between $f_{N, R}^{(s)}(t)$, the marginal of order $s$, and $\tilde{f}_{N, R}^{(s)}(t)$, the truncated marginals of order $s$, converges to 0 as $N$ goes to $\infty$ under the Boltzmann-Grad scaling $N \varepsilon^{d-1}=1$.

Thus, we finally have proved one of our main result:

Theorem 8.7. Consider the initial distribution $f_{N}^{0}$ defined in (2.1.1), the distribution of the tagged particle $f_{N}^{(1)}(t, x, v)$ converges in $\mathcal{D}^{\prime}\left(\mathbf{T}^{d} \times \mathbf{R}^{d}\right)$ as $N$ goes to $\infty$ under the Boltzmann-Grad scaling $N \varepsilon^{d-1}=1$ to $g_{R}(t, x, v)$ with initial data $g_{R}^{0}$ stated in (8.1.3) on a fixed $[0, t]$.

Finally, in order to prove the main theorem of this paper announced in Theorem 2.1, it remains to prove the convergence of Boltzmann with cut-off to Boltzmann without cut-off

\section{From Boltzmann with cut-off to Boltzmann without cut- off}

For $h_{R}$ satisfying the linear Boltzmann equation with cut-off, with initial data $\rho^{0}$, then the family $\left(h_{R}(t, z) M_{\beta}^{\otimes s}\left(V_{s}\right)\right)_{s \geq 1}$ is a solution to the Boltzmann with cut-off hierarchy. However by definition, $\left(g_{R}^{(s)}\right)_{s \geq 1}$ is already a solution too. Then by uniqueness of the hierarchy, $g_{R}(t, z)=h_{R}(t, z) M_{\beta}(v)$. Then, if we prove that $h_{R}$ solution of the Boltzmann equation with cut-off converges as $R$ goes to $\infty$ to $h$ solution of the Boltzmann equation without cut-off, we would have completed the proof of Theorem 2.1.

Proposition 9.1. For $\rho^{0}$ initial data, the solution $h_{R}$ of the Boltzmann equation with cut-off converges (up to an extraction of subsequence) in $L^{\infty}$ weak-* to a solution $h$ of the Boltzmann equation without cut-off. 
Proof. $h_{R}$ being a solution of the Boltzmann equation with cut-off, for $\varphi$ a test function, it satisfies:

$$
\begin{aligned}
& -\int_{0}^{T} \int_{\mathbf{T}} \int_{\mathbf{R}} h_{R}(t, x, v)\left(\partial_{t} \varphi+v \cdot \nabla_{x} \varphi\right) d v d x d t \\
& \quad-\int_{\mathbf{T}} \int_{\mathbf{R}} \rho^{0}(x, v) \varphi(0, x, v) d v d x \\
= & \int_{0}^{T} \int_{\mathbf{T}} \int_{\mathbf{R}} h_{R}(t, x, v) \int_{\mathbf{S}^{d-1} \times \mathbf{R}} R^{d-1}\left[\varphi(t, x, v)-\varphi\left(t, x, v^{* R}\right)\right] M_{\beta}\left(v_{1}\right)\left(\left(v-v_{1}\right) \cdot \nu\right)_{+} d v_{1} d \nu d v d x d t .
\end{aligned}
$$

Applying the maximum principle, we know that the sequence $h_{R}$ (up to an extraction of a subsequence) converges weak-* in $L^{\infty}$ to a function $h$. So we can pass to the limit on the left-hand side of (9.0.9).

Let us now deal with the right-hand side of (9.0.9) and study

$$
\left(\int_{\mathbf{S}^{d-1} \times \mathbf{R}} R^{d-1}\left[\varphi(t, x, v)-\varphi\left(t, x, v^{* R}\right)\right] M_{\beta}\left(v_{1}\right)\left(\left(v-v_{1}\right) \cdot \nu\right)_{+} d v_{1} d \nu\right)_{R>0}
$$

Actually, it is enough to study the difference between the two following terms

$$
\int_{\mathbf{S}^{d-1} \times \mathbf{R}} R^{d-1}\left[\varphi(t, x, v)-\varphi\left(t, x, v^{* R}\right)\right] M_{\beta}\left(v_{1}\right)\left(\left(v-v_{1}\right) \cdot \nu\right)_{+} d v_{1} d \nu
$$

and

$$
\int_{\mathbf{S}^{\mathbf{d}-1} \times \mathbf{R}}(2 R)^{d-1}\left[\varphi(t, x, v)-\varphi\left(t, x, v^{* 2 R}\right)\right] M_{\beta}\left(v_{1}\right)\left(\left(v-v_{1}\right) \cdot \nu\right)_{+} d v_{1} d \nu
$$

to conclude. Regarding this last term, let us split it in two terms by distinguishing two situations for the trajectories involved in it:

- one where the trajectories get into the sphere of radius $2 R$ without getting into the sphere of radius $R$, implying particles involved to be barely deviated for large $R$,

- one where the trajectories get into the sphere of radius $R$.

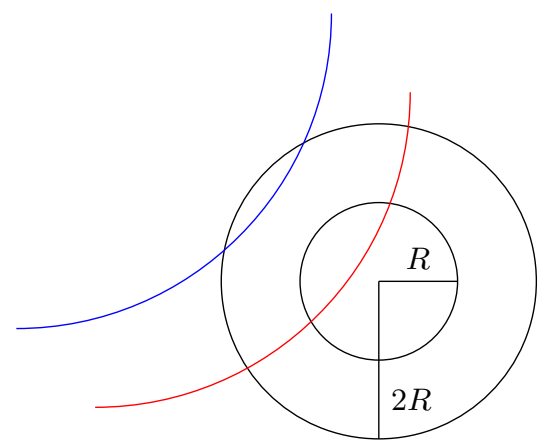

Figure 8. Representation of the two types of trajectories involved

Let us start with the first type of trajectories. The situation described is equivalent to work with the potential $\nabla \Phi \chi_{[R, 2 R[}$ where $\chi_{[R, 2 R[}$ is a regularization of $\mathbf{1}_{[R, 2 R[}$ such that it is equal to 1 on $[R, 2 R]$. Thus the evolution of the positions and the velocities is the following

$$
\left\{\begin{array}{l}
\frac{d x}{d t}=v, \\
\frac{d v}{d t}=-\nabla \Phi(x) \chi_{[R, 2 R[} .
\end{array}\right.
$$


Therefore integrating (9.0.13) and by assumptions on $\nabla \Phi$, we get

$$
\left|v(t)-v_{0}\right| \leq \exp \left(-\exp \left(12 \exp \left(\tilde{C}^{2} R^{2(d-1)} \log 2\right)\right)\right) t
$$

Moreover, using the bound of the maximal time of interaction stated in Proposition 4.2, we obtain that

$$
\left|v(t)-v_{0}\right| \leq \exp \left(-\exp \left(12 \exp \left(\tilde{C}^{2} R^{2(d-1)} \log 2\right)\right)\right) \frac{R^{2}}{\eta} .
$$

Thus, for $\tilde{\varphi}: \mathbf{R} \rightarrow \mathbf{R}$ a regular test function, we deduce from above that

$$
\left|\tilde{\varphi}\left(v^{* R}\right)-\tilde{\varphi}(v)\right| \leq C\|\nabla \tilde{\varphi}\|_{\infty} \exp \left(-\exp \left(12 \exp \left(\tilde{C}^{2} R^{2(d-1)} \log 2\right)\right)\right) \frac{R^{2}}{\eta} .
$$

Consequently, for the order of magnitude of our parameters stated in (7.0.7), (7.0.8) and (7.0.9), the part of the integral (9.0.11) corresponding to those trajectories converges to 0 .

Let us deal with the second type of trajectories. First of all, let us notice that the difference between the trajectory and the free transport in the annulus goes to 0 as $R$ grows. So the trajectory in the annulus can reasonably be approximated by straight lines and together with the inequality (9.0.15), we deduce that $v^{* R}-v^{* 2 R}$ converges to 0 from

$$
\left|v^{* R}-v^{* 2 R}\right| \leq C \exp \left(-\exp \left(12 \exp \left(\tilde{C}^{2} R^{2(d-1)} \log 2\right)\right)\right) \frac{R^{2}}{\eta} .
$$

Moreover, let us make a change of parametrization in (9.0.12) by parametrizing the the unit sphere by $(\theta, \psi)$ with $\psi \in \mathbf{S}^{d-2}$, we have

$$
d \nu=(\sin \theta)^{d-2} d \theta d \psi d v_{1} .
$$

Since the trajectories described in the second situation correspond to $\sin \theta<1 / 2$, we do the following change of variable $\sin \theta^{\prime}=2 \sin \theta$. Then, we get

$$
\begin{array}{r}
\int_{\left(\mathbf{S}^{\mathbf{d}-1} \times \mathbf{R}\right) \cap\{\sin \theta<1 / 2\}}(2 R)^{d-1}\left[\varphi(t, x, v)-\varphi\left(t, x, v^{* 2 R}\right)\right] M_{\beta}\left(v_{1}\right)\left(\left(v-v_{1}\right) \cdot \nu\right)_{+} d v_{1} d \nu \\
=\int_{\mathbf{S}^{\mathbf{d}-1} \times \mathbf{R}} R^{d-1}\left[\varphi(t, x, v)-\varphi\left(t, x, v^{* 2 R}\right)\right] M_{\beta}\left(v_{1}\right)\left(\left(v-v_{1}\right) \cdot \nu\right)_{+} d v_{1} d \nu .
\end{array}
$$

Thus finally,

$$
\begin{gathered}
\int_{\mathbf{S}^{\mathbf{d}-1} \times \mathbf{R}} R^{d-1}\left[\varphi(t, x, v)-\varphi\left(t, x, v^{* R}\right)\right] M_{\beta}\left(v_{1}\right)\left(\left(v-v_{1}\right) \cdot \nu\right)_{+} d v_{1} d \nu \\
-\int_{\left(\mathbf{S}^{\mathbf{d}-1} \times \mathbf{R}\right) \cap\{\sin \theta<1 / 2\}}(2 R)^{d-1}\left[\varphi(t, x, v)-\varphi\left(t, x, v^{* 2 R}\right)\right] M_{\beta}\left(v_{1}\right)\left(\left(v-v_{1}\right) \cdot \nu\right)_{+} d v_{1} d \nu \\
\quad=\int_{\mathbf{S}^{\mathbf{d}-1} \times \mathbf{R}} R^{d-1}\left[\varphi\left(t, x, v^{* 2 R}\right)-\varphi\left(t, x, v^{* R}\right)\right] M_{\beta}\left(v_{1}\right)\left(\left(v-v_{1}\right) \cdot \nu\right)_{+} d v_{1} d \nu .
\end{gathered}
$$

Using similarly as previously the regularity of $\varphi$ and the rate of convergence of $v^{* R}-v^{* 2 R}$ proves the convergence of the above term to 0 which concludes the proof. 


\section{Appendix}

\section{A A priori estimates}

In order to prove the a priori estimates, let us first establish the following lemma:

Lemma A.1. For $N>0, s \leq N$, in the Boltzmann-Grad scaling $N \varepsilon^{d-1}=1$, we have the following bound for $\overline{\mathcal{Z}}_{N}$ defined in $(2.1 .3)$

$$
1 \leq \overline{\mathcal{Z}}_{N}^{-1} \overline{\mathcal{Z}}_{N-s} \leq\left(1-\kappa_{d} R^{d} \varepsilon\right)^{-s} \exp \left(\beta s^{2}\left\|\Phi_{>}\right\|_{\infty}\right)
$$

where $\kappa_{d}$ denotes the volume of the unit ball in $\mathbf{R}^{d}$.

Proof. We have due to the nonnegativity of $\Phi$ that

$$
\begin{aligned}
\overline{\mathcal{Z}}_{s+1} & =\int_{\mathbf{T}^{d s}}\left(\int_{\mathbf{T}^{d}} \exp \left(-\beta \sum_{1 \leq i \leq s} \Phi\left(\frac{x_{i}-x_{s+1}}{\varepsilon}\right) d x_{s+1}\right)\right) \exp \left(-\beta \sum_{1 \leq k<l \leq s} \Phi\left(\frac{x_{k}-x_{l}}{\varepsilon}\right)\right) d X_{s} \\
& \leq \int_{\mathbf{T}^{d s}} \exp \left(-\beta \sum_{1 \leq k<l \leq s} \Phi\left(\frac{x_{k}-x_{l}}{\varepsilon}\right)\right) d X_{s}=\overline{\mathcal{Z}}_{s} .
\end{aligned}
$$

On the other hand,

$$
\begin{aligned}
& \overline{\mathcal{Z}}_{s+1} \geq \int_{\mathbf{T}^{d s}} \int_{\mathbf{T}^{d}} \exp \left(-\beta \sum_{1 \leq i \leq s} \Phi\left(\frac{x_{i}-x_{s+1}}{\varepsilon}\right)\right) \prod_{1 \leq i \leq s} \mathbf{1}_{\left|x_{i}-x_{s+1}\right|>R \varepsilon} d x_{s+1} \\
& \exp \left(-\beta \sum_{1 \leq k<l \leq s} \Phi\left(\frac{x_{k}-x_{l}}{\varepsilon}\right)\right) d X_{s} \\
& =\int_{\mathbf{T}^{d s}} \int_{\mathbf{T}^{d}} \exp \left(-\beta \sum_{1 \leq i \leq s} \Phi^{>}\left(\frac{x_{i}-x_{s+1}}{\varepsilon}\right)\right) \\
& \prod_{1 \leq i \leq s} \mathbf{1}_{\left|x_{k}-x_{s+1}\right|>R \varepsilon} d x_{s+1} \exp \left(-\beta \sum_{1 \leq k<l \leq s} \Phi\left(\frac{x_{k}-x_{l}}{\varepsilon}\right)\right) d X_{s} \\
& \geq \int_{\mathbf{T}^{d s}} \int_{\mathbf{T}^{d}} \exp \left(-\beta s\left\|\Phi^{>}\right\|_{\infty}\right) \\
& \prod_{1 \leq i \leq s} \mathbf{1}_{\left|x_{k}-x_{s+1}\right|>R \varepsilon} d x_{s+1} \exp \left(-\beta \sum_{1 \leq k<l \leq s} \Phi\left(\frac{x_{k}-x_{l}}{\varepsilon}\right)\right) d X_{s} \\
& \geq \exp \left(-\beta s\left\|\Phi^{>}\right\|_{\infty}\right) \int_{\mathbf{T}^{d s}}\left(\int_{\mathbf{T}^{d}} \prod_{1 \leq i \leq s} \mathbf{1}_{\left|x_{k}-x_{s+1}\right|>R \varepsilon} d x_{s+1}\right) \exp \left(-\beta \sum_{1 \leq k<l \leq s} \Phi\left(\frac{x_{k}-x_{l}}{\varepsilon}\right)\right) d X_{s} .
\end{aligned}
$$

However we know that

$$
\begin{aligned}
& \int_{\mathbf{T}^{d}} \prod_{1 \leq i \leq s} \mathbf{1}_{\left|x_{k}-x_{s+1}\right|>R \varepsilon} d x_{s+1} \geq \int_{\mathbf{T}^{d}}\left(1-\sum_{1 \leq i \leq s} \mathbf{1}_{\left|x_{k}-x_{s+1}\right| \leq R \varepsilon}\right) d x_{s+1} \\
& =1-\kappa_{d} s(R \varepsilon)^{d} \text {. }
\end{aligned}
$$

Therefore finally,

$$
\begin{aligned}
\overline{\mathcal{Z}}_{s+1} & \geq \exp \left(-\beta s\left\|\Phi^{>}\right\|_{\infty}\right) \overline{\mathcal{Z}}_{s}\left(1-\kappa_{d} s(R \varepsilon)^{d}\right) \\
& \geq \exp \left(-\beta s\left\|\Phi^{>}\right\|_{\infty}\right) \overline{\mathcal{Z}}_{s}\left(1-\kappa_{d} N(R \varepsilon)^{d}\right) \\
& =\exp \left(-\beta s\left\|\Phi^{>}\right\|_{\infty}\right) \overline{\mathcal{Z}}_{s}\left(1-\kappa_{d} R^{d} \varepsilon\right)
\end{aligned}
$$


and by induction

$$
\overline{\mathcal{Z}}_{N} \geq \exp \left(-\beta s^{2}\left\|\Phi^{>}\right\|_{\infty}\right) \overline{\mathcal{Z}}_{N-s}\left(1-\kappa_{d} R^{d} \varepsilon\right)^{s}
$$

which allow us to obtain the expected bounds.

We recall here the definitions for the truncated marginals $\tilde{f}_{N, R}^{(s)}$ and the term associated to clusters $\bar{f}_{N, R}^{(s)}$ :

$$
\tilde{f}_{N, R}^{(s)}\left(t, Z_{s}\right):=\int_{\mathbf{T}^{d(N-s)} \times \mathbf{R}^{d(N-s)}} f_{N}\left(t, Z_{s}, z_{s+1}, \ldots, z_{N}\right) \prod_{\substack{1 \leq i \leq s \\ s+1 \leq j \leq N}} \mathbf{1}_{\left\{\left|x_{i}-x_{j}\right|>R \varepsilon\right\}} d Z_{(s+1, N)}
$$

and

$$
\begin{aligned}
\bar{f}_{N, R}^{(s+1)}\left(t, Z_{s+1}\right):= & \int_{\mathbf{T}^{d(N-(s+1))} \times \mathbf{R}^{d(N-(s+1))}} f_{N}\left(t, Z_{N}\right) \\
& \left(\prod_{\substack{1 \leq k \leq s \\
s+2 \leq l \leq N}} \mathbf{1}_{\left|x_{k}-x_{l}\right|>R \varepsilon}\right)\left(1-\prod_{j=s+2}^{N} \mathbf{1}_{\left|x_{j}-x_{s+1}\right|>R \varepsilon}\right) d Z_{(s+2, N) .}
\end{aligned}
$$

Proposition A.1. For any fixed $N$, considering the initial data (2.1.1) for any $s \geq 1$, we have the following uniform bound (with respect to time)

$$
\sup _{t \geq 0}\left\|\tilde{f}_{N, R}^{(s)}(t)\right\|_{\varepsilon, s, \beta} \leq\left\|\rho^{0}\right\|_{\infty}\left(\frac{\beta}{2 \pi}\right)^{d s / 2}\left(1-\varepsilon \kappa_{d} R^{d}\right)^{-s} \exp \left(\beta s^{2}\left\|\Phi_{>R}\right\|_{\infty}\right)
$$

and

$$
\sup _{t \geq 0}\left\|\bar{f}_{N, R}^{(s)}(t)\right\|_{\varepsilon, s, \beta} \leq\left\|\rho^{0}\right\|_{\infty}\left(\frac{\beta}{2 \pi}\right)^{d s / 2}\left(1-\varepsilon \kappa_{d} R^{d}\right)^{-(s-1)} \exp \left(\beta s^{2}\left\|\Phi_{>R}\right\|_{\infty}\right) R^{d} \varepsilon .
$$

Proof. We have that

$$
f_{N}^{0}\left(Z_{N}\right)\left(t, Z_{N}\right)=M_{N, \beta}\left(Z_{N}\right) \rho^{0}\left(x_{1}\right) \leq M_{N, \beta}\left(Z_{N}\right)\left\|\rho^{0}\right\|_{\infty} .
$$

Applying the maximum principle for the Liouville equation, $M_{N, \beta}$ being a stationary solution, we obtain for all $t \in \mathbf{R}$

$$
f_{N}\left(t, Z_{N}\right) \leq M_{N, \beta}\left(Z_{N}\right)\left\|\rho^{0}\right\|_{\infty} .
$$

In order to obtain the bounds, we need to multiply (A.0.27) by the appropriate terms and integrate.

First, we are interested in the truncated marginals $\tilde{f}_{N, R}^{(s)}$

$$
\begin{aligned}
& \left|\tilde{f}_{N, R}^{(s)}\left(t, Z_{s}\right)\right|=\int_{\mathbf{T}^{d(N-s)} \times \mathbf{R}^{d(N-s)}} f_{N}\left(t, Z_{N}\right) \prod_{\substack{1 \leq i \leq s \\
s+1 \leq j \leq N}} \mathbf{1}_{\left\{\left|x_{i}-x_{j}\right|>R \varepsilon\right\}} d Z_{(s+1, N)} \\
& \leq \frac{1}{\overline{\mathcal{Z}}_{N}} \int_{\mathbf{T}^{d(N-s)} \times \mathbf{R}^{d(N-s)}}\left\|\rho^{0}\right\|_{\infty}\left(\frac{\beta}{2 \pi}\right)^{d N / 2} \exp \left(-\beta H_{N}\left(Z_{N}\right)\right) \prod_{\substack{1 \leq i \leq s \\
s+1 \leq j \leq N}} \mathbf{1}_{\left\{\left|x_{i}-x_{j}\right|>R \varepsilon\right\}} d Z_{(s+1, N)} \\
& \leq \frac{1}{\overline{\mathcal{Z}}_{N}}\left(\frac{\beta}{2 \pi}\right)^{d s / 2}\left\|\rho^{0}\right\|_{\infty} \exp \left(-\beta H_{s}\left(Z_{s}\right)\right) \int_{\mathbf{T}^{d(N-s)}} \exp \left(-\beta \sum_{s+1 \leq i<j \leq N} \Phi\left(\frac{x_{i}-x_{j}}{\varepsilon}\right)\right) d X_{(s+1, N)} \\
& \leq \overline{\mathcal{Z}}_{N}^{-1} \overline{\mathcal{Z}}_{N-s}\left(\frac{\beta}{2 \pi}\right)^{d s / 2}\left\|\rho^{0}\right\|_{\infty} \exp \left(-\beta H_{s}^{<}\left(Z_{s}\right)\right) .
\end{aligned}
$$


using the fact that $\Phi^{<} \leq \Phi$ and $\Phi$ is nonnegative. Hence

$$
\left|\tilde{f}_{N, R}^{(s)}\left(t, Z_{s}\right) \exp \left(\beta H_{s}^{<}\left(Z_{s}\right)\right)\right| \leq\left\|\rho^{0}\right\|_{\infty}\left(\frac{\beta}{2 \pi}\right)^{d s / 2} \overline{\mathcal{Z}}_{N}^{-1} \overline{\mathcal{Z}}_{N-s}
$$

and finally

$$
\sup _{t \geq 0}\left\|\tilde{f}_{N, R}^{(s)}(t)\right\|_{\varepsilon, s, \beta} \leq\left\|\rho^{0}\right\|_{\infty}\left(\frac{\beta}{2 \pi}\right)^{d s / 2} \overline{\mathcal{Z}}_{N}^{-1} \overline{\mathcal{Z}}_{N-s}
$$

We perform the same computations for $\bar{f}_{N, R}^{(s)}$

$$
\begin{aligned}
& \left|\bar{f}_{N, R}^{(s)}\left(t, Z_{s}\right)\right|=\int_{\mathbf{T}^{d(N-s)} \times \mathbf{R}^{d(N-s)}} f_{N}\left(t, Z_{N}\right)\left(\prod_{\substack{1 \leq k \leq s-1 \\
s+1 \leq l \leq N}} \mathbf{1}_{\left|x_{k}-x_{l}\right|>R \varepsilon}\right)\left(1-\prod_{j=s+1}^{N} \mathbf{1}_{\left|x_{j}-x_{s}\right|>R \varepsilon}\right) d Z_{(s+1, N)} \\
& \leq \frac{1}{\overline{\mathcal{Z}}_{N}} \int_{\mathbf{T}^{d(N-s)} \times \mathbf{R}^{d(N-s)}}\left\|\rho^{0}\right\|_{\infty}\left(\frac{\beta}{2 \pi}\right)^{d N / 2} \exp \left(-\beta H_{N}\left(Z_{N}\right)\right)\left(1-\prod_{j=s+1}^{N} \mathbf{1}_{\left|x_{j}-x_{s}\right|>R \varepsilon}\right) \\
& \leq \quad \frac{1}{\overline{\mathcal{Z}}_{N}}\left\|\rho^{0}\right\|_{\infty}\left(\frac{\beta}{2 \pi}\right)^{d s / 2} \exp \left(-\beta H_{s}^{<}\left(Z_{s}\right)\right) \\
& \sum_{j=s+1}^{N} \int_{\mathbf{T}^{d(N-(s+1))}}\left(\int_{\mathbf{T}^{d}}\left(\mathbf{1}_{\left|x_{j}-x_{s}\right| \leq R \varepsilon}\right) d x_{j}\right) \exp \left(\sum_{\substack{s+1 \leq k<l \leq N \\
\bar{k}, l \neq j}} \Phi\left(\frac{x_{k}-x_{l}}{\varepsilon}\right)\right) \\
& \leq\left\|\rho^{0}\right\|_{\infty}\left(\frac{\beta}{2 \pi}\right)^{d s / 2} R^{d} \varepsilon \overline{\mathcal{Z}}_{N}^{-1} \overline{\mathcal{Z}}_{N-(s+1)} \exp \left(-\beta H_{s}^{<}\left(Z_{s}\right)\right) .
\end{aligned}
$$

Hence

$$
\left|\bar{f}_{N, R}^{(s)}\left(t, Z_{s}\right) \exp \left(\beta H_{s}^{<}\left(Z_{s}\right)\right)\right| \leq\left\|\rho^{0}\right\|_{\infty}\left(\frac{\beta}{2 \pi}\right)^{d s / 2} R^{d} \varepsilon \overline{\mathcal{Z}}_{N}^{-1} \overline{\mathcal{Z}}_{N-(s+1)}
$$

and finally

$$
\sup _{t \geq 0}\left\|\bar{f}_{N, R}^{(s)}(t)\right\|_{\varepsilon, s, \beta} \leq\left\|\rho^{0}\right\|_{\infty}\left(\frac{\beta}{2 \pi}\right)^{d s / 2} R^{d} \varepsilon \overline{\mathcal{Z}}_{N}^{-1} \overline{\mathcal{Z}}_{N-(s+1)}
$$

and we get the desired bound using again Lemma A.1.

\section{B Asymptotical factorisation of the initial data}

Proposition B.1. Given $\beta>0, s \geq 1$, the truncated marginal of order $s$

$$
\tilde{M}_{N, \beta}^{(s)}:=\int M_{N, \beta}\left(Z_{N}\right) \prod_{\substack{1 \leq i \leq s \\ s+1 \leq j \leq N}} \mathbf{1}_{\left\{\left|x_{i}-x_{j}\right|>R \varepsilon\right\}} d Z_{(s+1, N)}
$$

converges as $N$ goes to $\infty$, under the Boltzmann-Grad scaling $N \varepsilon^{d-1}=1$ to the Maxwell distribution $M_{\beta}^{\otimes s}$ where $M_{\beta}^{\otimes s}:=\prod_{i=1}^{s} M_{\beta}\left(v_{i}\right)$ and $M_{\beta}(v):=\left(\frac{\beta}{2 \pi}\right)^{d / 2} \exp \left(-\frac{\beta}{2}|v|^{2}\right)$. 
Proof. We have that

$$
\begin{aligned}
& \tilde{M}_{N, \beta}^{(s)}\left(Z_{s}\right)=\frac{1}{\overline{\mathcal{Z}}_{N}}\left(\frac{\beta}{2 \pi}\right)^{d N / 2} \int \exp \left(-\frac{\beta}{2 \pi} \sum_{i=1}^{N}\left|v_{i}\right|^{2}-\beta \sum_{1 \leq i<j \leq N} \Phi\left(\frac{x_{i}-x_{j}}{\varepsilon}\right)\right) \\
& \prod_{\left.1 \leq x_{i}-x_{j} \mid>R \varepsilon\right\}} d Z_{(s+1, N)} \\
& \begin{array}{c}
1 \leq i \leq s \\
s+1 \leq j \leq N \\
\end{array} \\
& =\frac{1}{\overline{\mathcal{Z}}_{N}}\left(\frac{\beta}{2 \pi}\right)^{d s / 2} \exp \left(-\frac{\beta}{2 \pi} \sum_{i=1}^{s}\left|v_{i}\right|^{2}\right) \exp \left(-\beta \sum_{1 \leq i<j \leq s} \Phi\left(\frac{x_{i}-x_{j}}{\varepsilon}\right)\right) \\
& \int \exp \left(-\beta \sum_{s+1 \leq i<j \leq N} \Phi\left(\frac{x_{i}-x_{j}}{\varepsilon}\right)\right) \exp \left(-\beta \sum_{i^{\prime} \leq s<j^{\prime}} \Phi\left(\frac{x_{i}^{\prime}-x_{j}^{\prime}}{\varepsilon}\right)\right) \\
& \prod_{\substack{1 \leq k \leq s \\
s+1 \leq l \leq N}} \mathbf{1}_{\left\{\left|x_{k}-x_{l}\right|>R \varepsilon\right\}} d X_{(s+1, N)} \\
& =\frac{1}{\overline{\mathcal{Z}}_{N}}\left(\frac{\beta}{2 \pi}\right)^{d s / 2} \exp \left(-\frac{\beta}{2 \pi} \sum_{i=1}^{s}\left|v_{i}\right|^{2}\right) \exp \left(-\beta \sum_{1 \leq i<j \leq s} \Phi\left(\frac{x_{i}-x_{j}}{\varepsilon}\right)\right) \\
& {\left[\overline{\mathcal{Z}}_{N-s}-\overline{\mathcal{Z}}_{(s+1, N)}^{b}\right]}
\end{aligned}
$$

because of the symmetry, with

$$
\begin{gathered}
\overline{\mathcal{Z}}_{(s+1, N)}^{b}:=\int \exp \left(-\beta \sum_{s+1 \leq i<j \leq N} \Phi\left(\frac{x_{i}-x_{j}}{\varepsilon}\right)\right)\left(1-\exp \left(-\beta \sum_{\substack{1 \leq k \leq s \\
s+1 \leq l \leq N}} \mathbf{1}_{\substack{\left.i^{\prime} \leq s<j^{\prime} \\
s\left|x_{k}-x_{l}\right|>R \varepsilon\right\}}} \Phi\left(\frac{x_{i}^{\prime}-x_{j}^{\prime}}{\varepsilon}\right)\right)\right) \\
\prod_{(s+1, N)} .
\end{gathered}
$$

Yet,

$$
\begin{gathered}
\frac{\overline{\mathcal{Z}}_{(s+1, N)}^{b}}{\overline{\mathcal{Z}}_{N}} \leq \frac{1}{\overline{\mathcal{Z}}_{N}} \int \exp \left(-\beta \sum_{s+1 \leq i<j \leq N} \Phi\left(\frac{x_{i}-x_{j}}{\varepsilon}\right)\right)\left(1-\exp \left(-\beta s^{2}\left\|\Phi^{>}\right\|_{\infty}\right)\right) \\
\prod_{\substack{1 \leq k \leq s \\
s+1 \leq l \leq N}} \mathbf{1}_{\left\{\left|x_{k}-x_{l}\right|>R \varepsilon\right\}} d X_{(s+1, N)}
\end{gathered}
$$

because of the monoticity of $\Phi$ in Assumption 4.1. Finally,

$$
\frac{\overline{\mathcal{Z}}_{(s+1, N)}^{b}}{\overline{\mathcal{Z}}_{N}} \leq \frac{\overline{\mathcal{Z}}_{N-s}}{\overline{\mathcal{Z}}_{N}}\left(1-\exp \left(-\beta s^{2}\left\|\Phi^{>}\right\|_{\infty}\right)\right)
$$

using again the symmetry.

Because of the Assumption 4.1, Lemma A.1 allow us to prove that $\frac{\overline{\mathcal{Z}}_{N-s}}{\overline{\mathcal{Z}}_{N}}$ and $\frac{\overline{\mathcal{Z}}_{N-s-1}}{\overline{\mathcal{Z}}_{N}}$ both converge to 1 as $N$ goes to $\infty$. Using the decreasing assumption on the potential leads to the statement that $\frac{\overline{\mathcal{Z}}_{(s+1, N)}^{\mathrm{b}}}{\overline{\mathcal{Z}}_{N}}$ converges to 0 as $N$ goes to $\infty$. 
So finally,

$$
\begin{aligned}
& \left|\tilde{M}_{N, \beta}^{(s)}\left(Z_{s}\right)-M_{\beta}^{\otimes s}\left(V_{s}\right)\right|=\left(\frac{\beta}{2 \pi}\right)^{d s / 2} \exp \left(-\frac{\beta}{2} \sum_{i=1}^{s}\left|v_{i}\right|^{2}\right) \\
& \left(\frac{\overline{\mathcal{Z}}_{N-s}}{\overline{\mathcal{Z}}_{N}} \exp \left(-\beta \sum_{1 \leq i<j \leq s} \Phi\left(\frac{x_{i}-x_{j}}{\varepsilon}\right)\right)-1-\frac{\overline{\mathcal{Z}}_{(s+1, N)}^{b}}{\overline{\mathcal{Z}}_{N}} \exp \left(-\beta \sum_{1 \leq i<j \leq s} \Phi\left(\frac{x_{i}-x_{j}}{\varepsilon}\right)\right)\right)
\end{aligned}
$$

and using that by assumptions $\Phi$ vanishes at infinity, and the statements of above about $\frac{\overline{\mathcal{Z}}_{N-s}}{\overline{\mathcal{Z}}_{N}}$ and $\frac{\overline{\mathcal{Z}}_{\left(s_{+1, N)}\right.}^{b}}{\overline{\mathcal{Z}}_{N}}$, we conclude that this difference converges to 0 as $N$ goes to $\infty$ which is precisely the assertion of the proposition.

Corollary B.2. For the initial data $f_{N}$ given in (2.1.1), the truncated marginal of order $s$

$$
\tilde{f}_{N}^{0(s)}:=\int f_{N}^{0}\left(Z_{N}\right) \prod_{\substack{1 \leq i \leq s \\ s+1 \leq j \leq N}} \mathbf{1}_{\left\{\left|x_{i}-x_{j}\right|>R \varepsilon\right\}} d Z_{(s+1, N)}=\rho^{0}\left(x_{1}\right) \tilde{M}_{N, \beta}^{(s)}\left(Z_{s}\right)
$$

converges as $N$ goes to $\infty$, under the Boltzmann-Grad scaling $N \varepsilon^{d-1}=1$, to the function $g^{0(s)}$ where $g^{0(s)}$ is defined by $g^{0(s)}\left(Z_{s}\right):=\rho^{0}\left(x_{1}\right) M_{\beta}^{\otimes s}\left(V_{s}\right)$.

The proof is straightforward using the fact that $\rho^{0}$ is bounded.

Proposition B.3. For the initial data $f_{N}^{0}$ given in (2.1.1), for all $t \geq 0$, the difference between $f_{N, R}^{(s)}(t)$, the marginal of order $s$, and $\tilde{f}_{N, R}^{(s)}(t)$, the truncated marginals of order $s$, converges when $N$ goes to $\infty$ under the Boltzmann-Grad scaling $N \varepsilon^{d-1}=1$ to 0 .

Proof. We have that

$$
\begin{aligned}
& \left|f_{N, R}^{(s)}\left(t, Z_{s}\right)-\tilde{f}_{N, R}^{(s)}\left(t, Z_{s}\right)\right|=\int f_{N, R}\left(t, Z_{N}\right)\left(1-\prod_{\substack{1 \leq k \leq s \\
s+1 \leq l \leq N}} \mathbf{1}_{\left\{\left|x_{k}-x_{l}\right|>R \varepsilon\right\}}\right) d Z_{(s+1, N)} \\
& \leq\left\|\rho^{0}\right\|_{\infty} \int M_{N, \beta}\left(t, Z_{N}\right)\left(1-\prod_{\substack{1 \leq k \leq s \\
s+1 \leq l \leq N}} \mathbf{1}_{\left\{\left|x_{k}-x_{l}\right|>R \varepsilon\right\}}\right) d Z_{(s+1, N)} \\
& \leq \frac{\left\|\rho^{0}\right\|_{\infty}}{\overline{\mathcal{Z}}_{N}}\left(\frac{\beta}{2}\right)^{d s / 2} \exp \left(-\frac{\beta}{2} \sum_{i=1}^{s}\left|v_{i}\right|^{2}\right) \exp \left(-\beta \sum_{1 \leq i<j \leq s} \Phi\left(\frac{x_{i}-x_{j}}{\varepsilon}\right)\right) \\
& \int\left(1-\prod_{\substack{1 \leq k \leq s \\
s+1 \leq l \leq N}} \mathbf{1}_{\left\{\left|x_{k}-x_{l}\right|>R \varepsilon\right\}}\right) \exp \left(-\beta \sum_{s+1 \leq i<j \leq N} \Phi\left(\frac{x_{i}-x_{j}}{\varepsilon}\right)\right) \\
& \exp \left(-\beta \sum_{i^{\prime} \leq s<j^{\prime}} \Phi\left(\frac{x_{i}^{\prime}-x_{j}^{\prime}}{\varepsilon}\right)\right) d X_{(s+1, N)} \\
& \leq \frac{\left\|\rho^{0}\right\|_{\infty}}{\overline{\mathcal{Z}}_{N}}\left(\frac{\beta}{2 \pi}\right)^{d s / 2} \exp \left(-\beta H_{s}\left(Z_{s}\right)\right) \\
& (N-s) \sum_{i=1}^{s} \int \mathbf{1}_{\left|x_{k}-x_{s+1}\right| \leq R \varepsilon} \exp \left(-\beta \sum_{s+1 \leq i<j \leq N} \Phi\left(\frac{x_{i}-x_{j}}{\varepsilon}\right)\right) d X_{(s+1, N)}
\end{aligned}
$$




$$
\begin{aligned}
& \leq \frac{\left\|\rho^{0}\right\|_{\infty}}{\overline{\mathcal{Z}}_{N}}\left(\frac{\beta}{2 \pi}\right)^{d s / 2} \exp \left(-\beta H_{s}\left(Z_{s}\right)\right) \\
& \quad(N-s) \sum_{i=1}^{s} \int \exp \left(-\beta \sum_{s+2 \leq i<j \leq N} \Phi\left(\frac{x_{i}-x_{j}}{\varepsilon}\right)\right) \int \mathbf{1}_{\left|x_{k}-x_{s+1}\right| \leq R \varepsilon} d x_{s+1} d X_{(s+2, N)} \\
& \leq \frac{\left\|\rho^{0}\right\|_{\infty}}{\overline{\mathcal{Z}}_{N}}\left(\frac{\beta}{2 \pi}\right)^{d s / 2} \exp \left(-\beta H_{s}\left(Z_{s}\right)\right)(N-s) s(R \varepsilon)^{d} \kappa_{d} \overline{\mathcal{Z}}_{N-s-1} \\
& \leq \frac{\overline{\mathcal{Z}}_{N-s-1}}{\overline{\mathcal{Z}}_{N}}\left\|\rho^{0}\right\|_{\infty}\left(\frac{\beta}{2 \pi}\right)^{d s / 2} \exp \left(-\beta H_{s}\left(Z_{s}\right)\right) s R^{d} \varepsilon \kappa_{d}
\end{aligned}
$$

which concludes the proof.

\section{Continuity estimates}

We recall the definition of the weighted norms: for $f_{k}$ defined on $\mathbf{T}^{d k} \times \mathbf{R}^{d k}$,

$$
\left\|f_{k}\right\|_{\varepsilon, k, \alpha}:=\sup _{Z_{k} \in \mathbf{T}^{d k} \times \mathbf{R}^{d k}}\left|f_{k}\left(Z_{k}\right) \exp \left(\alpha H_{k}^{<}\left(Z_{k}\right)\right)\right|<\infty
$$

Lemma C.1. There is a constant $C_{d}$ depending only on $d$ such that for all $s, n \in \mathbf{N}, t \geq 0$, the oeprators $|Q|_{s, s+n}(t)$ satisfy the following continuity estimates: for all $f_{s+n} \in X_{\varepsilon, s+n, \alpha},|Q|_{s, s+n}(t) f_{s+n}$ belongs to $X_{\varepsilon, s, \frac{\alpha}{2}}$ and

$$
\left\||Q|_{s, s+n}(t) f_{s+n}\right\|_{\varepsilon, s, \frac{\alpha}{2}} \leq e^{s-1}\left(\frac{C_{d} R^{d-1} t}{\alpha^{\frac{d+1}{2}}}\right)^{n}\left\|f_{s+n}\right\|_{\varepsilon, s+n, \alpha} .
$$

Proof. First of all, let us notice that the transport operators preserve the weighted norms. Indeed, we have the following identities

$$
\left\|\mathcal{S}_{k}(t) f_{k}\right\|_{\varepsilon, k, \alpha}=\left\|f_{k}\right\|_{\varepsilon, k, \alpha} .
$$

Second, let us obtain some bounds for the collision operators in the Boltzmann-Grad scaling $N \varepsilon^{d-1}=1$. We have

$$
\begin{aligned}
&\left|\mathcal{C}_{s, s+1} f_{s+1}\left(Z_{s}\right)\right|=\mid(N-s) \sum_{i=1}^{s} \int_{S_{R \varepsilon}\left(x_{i}\right) \times \mathbf{R}^{d}}\left(\prod_{\substack{j=1 \\
j \neq i}}^{s} \mathbf{1}_{\left|x_{j}-x_{s+1}\right|>R \varepsilon} f_{s+1}\left(Z_{s+1}\right) d \sigma_{i}\left(x_{s+1}\right) d v_{s+1} \mid\right. \\
& \leq(N-s) \sum_{i=1}^{s} \int_{S_{R \varepsilon}\left(x_{i}\right) \times \mathbf{R}^{d}}\left(\left|v_{s+1}\right|+\left|v_{i}\right|\right) f_{s+1}\left(Z_{s+1}\right) d \sigma_{i}\left(x_{s+1}\right) d v_{s+1} \\
& \leq R^{d-1}\left\|f_{s+1}\right\|_{\varepsilon, s+1, \alpha} \sum_{i=1}^{s} \int_{\mathbf{R}^{d}}\left(\left|v_{s+1}\right|+\left|v_{i}\right|\right) \exp \left(-\alpha H_{s+1}^{<}\left(Z_{s+1}\right)\right) d v_{s+1} \\
& \leq R^{d-1}\left\|f_{s+1}\right\|_{\varepsilon, s+1, \alpha} \exp \left(-\frac{\alpha}{2} H_{s}^{<}\left(Z_{s}\right)\right) \\
& \leq R^{d-1}\left\|f_{s+1}\right\|_{\varepsilon, s+1, \alpha} \exp \left(-\frac{\alpha}{2} H_{\mathbf{R}^{d}}\left(\left|v_{s+1}\right|+\left|v_{i}\right|\right) \exp \left(-\frac{\alpha}{2} H_{s+1}^{<}\left(Z_{s+1}\right)\right) d v_{s+1}\right) \\
& \sum_{i=1}^{s} \int_{\mathbf{R}^{d}}\left(\left|v_{s+1}\right|+\left|v_{i}\right|\right) \exp \left(-\frac{\alpha}{2} \sum_{k=1}^{s+1}\left|v_{k}\right|^{2}\right) d v_{s+1} .
\end{aligned}
$$

We know by a direct calculation that

$$
\int_{\mathbf{R}^{d}}\left(\left|v_{s+1}\right|+\left|v_{i}\right|\right) \exp \left(-\frac{\alpha}{2} \sum_{k=1}^{s+1}\left|v_{k}\right|^{2}\right) d v_{s+1} \leq C_{d} \alpha^{-d / 2}\left(\alpha^{-1 / 2}+\left|v_{i}\right|\right) \exp \left(-\frac{\alpha}{2} \sum_{k=1}^{s}\left|v_{k}\right|^{2}\right) .
$$


So in the end,

$$
\begin{aligned}
&\left|\mathcal{C}_{s, s+1} f_{s+1}\left(Z_{s}\right)\right| \leq C_{d} R^{d-1} \alpha^{-d / 2}\left(s \alpha^{-1 / 2}+\sum_{i=1}^{s}\left|v_{i}\right|\right) \exp \left(-\frac{\alpha}{2} \sum_{k=1}^{s}\left|v_{k}\right|^{2}\right) \\
& \exp \left(-\frac{\alpha}{2} H_{s}^{<}\left(Z_{s}\right)\right)\left\|f_{s+1}\right\|_{\varepsilon, s+1, \alpha} .
\end{aligned}
$$

Finally, we pile together those inequalities, dispatching the first exponential above evenly on each occurrence of a collision term. Noticing that by Cauchy-Schwarz inequality, we get that

$$
\sum_{1 \leq i \leq k}\left|v_{i}\right| \exp \left(-\frac{\alpha}{4 n} \sum_{1 \leq j \leq k}\left|v_{j}\right|^{2}\right) \leq \sqrt{\frac{2}{e \alpha}}(s+n-1)
$$

and we are able to establish that each collision operator can be bounded by the term $C_{d} R^{d-1} \alpha^{(d+1) / 2}$ $(s+n-1)$ with a loss on the exponential weight. By integrating with respect to time, we obtain a factor $t^{n} / n$ !. All together with the Stirling formula, we are able to obtain the following bound

$$
t^{n} \frac{(s+n-1)^{n}}{n !} \leq t^{n} \exp (s+n-1)
$$

which allow us to conclude to the statement.

Acknowledgment. The author is deeply grateful to Laure Saint-Raymond for many helpful discussions.

\section{References}

[1] R. Alexandre. Remarks on 3D Boltzmann linear equation without cutoff. Transport Theory and Statistical Physics, 28(5):433-473, 1999.

[2] R. Alexandre, Y. Morimoto, S. Ukai, C.-J. Xu, and T. Yang. Global Existence and Full Regularity of the Boltzmann Equation Without Angular Cutoff. Communications in Mathematical Physics, 304(2):513-581, 2011.

[3] R. Alexandre, Y. Morimoto, S. Ukai, C.-J. Xu, and T. Yang. The Boltzmann equation without angular cutoff in the whole space: II, Global existence for hard potential. Analysis and Applications, 09(02):113-134, 2011.

[4] R. Alexandre, Y. Morimoto, S. Ukai, C.-J. Xu, and T. Yang. The Boltzmann equation without angular cutoff in the whole space: I, Global existence for soft potential. Journal of Functional Analysis, 262(3):915 - 1010, 2012.

[5] R. Alexandre and C. Villani. On The Boltzmann Equation For Long-Range Interactions. Comm. Pure Appl. Math, 55:30-70, 2002.

[6] A. V. Boblylev, M. Pulvirenti, and C. Saffirio. From Particle Systems to the Landau Equation: A Consistency Result. Communications in Mathematical Physics, 319(3):683-702, 2012.

[7] T. Bodineau, I. Gallagher, and L. Saint-Raymond. The Brownian motion as the limit of a deterministic system of hard-spheres. Inventiones mathematicae, 203(2):493-553, 2015.

[8] C. Cercignani. The Boltzmann equation and its application. Springer Verlag, 1988.

[9] L. Desvillettes and M. Pulvirenti. The linear Boltzmann equation for long-range forces: a derivation from particles system. Mathematical Models and Methods in Applied Sciences, 09(08):1123-1145, 1999. 
[10] I. Gallagher, L. Saint-Raymond, and B. Texier. From Newton to Boltzmann: Hard Spheres and Short-range Potentials. Zurich Lectures in Advanced Mathematics. European Mathematical Society, 2013.

[11] H. Grad. Principles of the Kinetic Theory of Gases. Handbuch der Physik, 12:205-294, 1958.

[12] P. T. Gressman, R. M. Strain, and R. V. Kadison. Global classical solutions of the Boltzmann equation with long-range interactions. Proceedings of the National Academy of Sciences of the United States of America, 107(13):5744-5749, 2010.

[13] F. King. BBGKY hierarchy for positive potentials. PhD thesis, Dept. Mathematics, Univ. California, Berkeley, 1975.

[14] O. E. Lanford. Time evolution of large classical systems. In J. Moser, editor, Dynamical Systems, Theory and Applications, volume 38 of Lecture Notes in Physics, pages 1-111. Springer Berlin Heidelberg, 1975.

[15] M. Pulvirenti, C. Saffirio, and S. Simonella. On the validity of the Boltzmann equation for short range potentials. Reviews in Mathematical Physics, 26(02):1450001, 2014.

[16] H. Spohn. Large scale dynamics of interacting particles. Springer Science \& Business Media, 2012.

Nathalie Ayi

Laboratory J.A. Dieudonné

UMR CNRS-UNS $N^{0} 7351$

University of Nice Sophia-Antipolis

Parc Valrose

06108 Nice Cedex 2

France

Member of the COFFEE team (INRIA)

e-mail: nathalie.ayi@unice.fr 